\title{
A low-energy perspective on the minimal left-right symmetric model
}

\author{
W. Dekens, ${ }^{a}$ L. Andreoli, ${ }^{b}$ J. de Vries, ${ }^{c, d}$ E. Mereghetti ${ }^{e}$ and F. Oosterhof ${ }^{f}$ \\ ${ }^{a}$ Department of Physics, University of California at San Diego, \\ La Jolla, CA 92093-0319, U.S.A. \\ ${ }^{b}$ Department of Physics, Washington University in Saint Louis, \\ Saint Louis, MO 63130, U.S.A. \\ ${ }^{c}$ Institute for Theoretical Physics Amsterdam and Delta Institute for Theoretical Physics, \\ University of Amsterdam, \\ Science Park 904, 1098 XH Amsterdam, The Netherlands \\ ${ }^{d}$ Nikhef, Theory Group, \\ Science Park 105, 1098 XG, Amsterdam, The Netherlands \\ e Theoretical Division, Los Alamos National Laboratory, \\ Los Alamos, NM 87545, U.S.A. \\ ${ }^{f}$ Van Swinderen Institute for Particle Physics and Gravity, University of Groningen, \\ 9747 AG Groningen, The Netherlands \\ E-mail: wdekens@physics.ucsd.edu, landreoli@wustl.edu, \\ j.devries4@uva.nl, emereghetti@lanl.gov, f.oosterhof@rug.nl
}

ABSTRACT: We perform a global analysis of the low-energy phenomenology of the minimal left-right symmetric model (mLRSM) with parity symmetry. We match the mLRSM to the Standard Model Effective Field Theory Lagrangian at the left-right-symmetry breaking scale and perform a comprehensive fit to low-energy data including mesonic, neutron, and nuclear $\beta$-decay processes, $\Delta F=1$ and $\Delta F=2$ CP-even and -odd processes in the bottom and strange sectors, and electric dipole moments (EDMs) of nucleons, nuclei, and atoms. We fit the Cabibbo-Kobayashi-Maskawa and mLRSM parameters simultaneously and determine a lower bound on the mass of the right-handed $W_{R}$ boson. In models where a Peccei-Quinn mechanism provides a solution to the strong CP problem, we obtain $M_{W_{R}} \gtrsim 5.5 \mathrm{TeV}$ at $95 \%$ C.L. which can be significantly improved with nextgeneration EDM experiments. In the $P$-symmetric mLRSM without a Peccei-Quinn mechanism we obtain a more stringent constraint $M_{W_{R}} \gtrsim 17 \mathrm{TeV}$ at $95 \%$ C.L., which is difficult to improve with low-energy measurements alone. In all cases, the additional scalar fields of the mLRSM are required to be a few times heavier than the right-handed gauge bosons. We consider a recent discrepancy in tests of first-row unitarity of the CKM matrix. 
We find that, while $\mathrm{TeV}$-scale $W_{R}$ bosons can alleviate some of the tension found in the $V_{u d, u s}$ determinations, a solution to the discrepancy is disfavored when taking into account other low-energy observables within the mLRSM.

KEywords: Beyond Standard Model, CP violation, Effective Field Theories

ArXIV EPrint: 2107.10852 


\section{Contents}

1 Introduction 1

2 Minimal left-right models $\quad 3$

2.1 Particle content 3

2.2 Symmetry breaking 4

2.3 Left-right symmetries 5

2.4 Strong CP problem and $P$ symmetry 6

3 Matching and renormalization group equations $\quad 7$

3.1 Matching conditions at $\mu=M_{W_{R}} \quad 9$

$\begin{array}{lll}3.2 & \text { Renormalization group equations below } M_{W_{R}} & 11\end{array}$

$\begin{array}{ll}3.3 \text { Matching at } \mu=m_{W} & 12\end{array}$

$\begin{array}{lll}3.4 & \text { Renormalization group equations below } \mu=m_{W} & 13\end{array}$

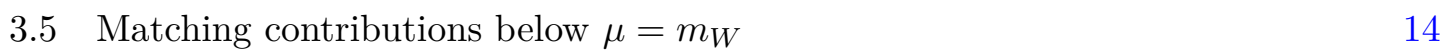

$\begin{array}{lll}3.6 & \text { Summary } & 15\end{array}$

4 The CP-violating chiral Lagrangian $\quad 16$

4.1 Vacuum alignment and the Peccei-Quinn mechanism 17

$\begin{array}{lll}4.2 & \text { CP-odd pion-nucleon interactions } & 22\end{array}$

5 Observables $\quad \mathbf{2 3}$

$\begin{array}{lll}5.1 & \text { Leptonic and semileptonic decays } & 25\end{array}$

5.2 Hadronic $\Delta S=1$ and $\Delta B=1$ charged-current processes 27

$5.3 \Delta F=2$ processes 28

$\begin{array}{lll}\text { 5.3.1 } & B-\bar{B} \text { oscillations } & 28\end{array}$

$\begin{array}{lll}\text { 5.3.2 } \Delta m_{K} \text { and } \varepsilon_{K} & 29\end{array}$

$5.4 \Delta F=0$ observables: electric dipole moments 32

5.4.1 Nucleon EDMs 32

5.4.2 Nuclear and atomic EDMs 33

6 Results $\quad 34$

6.1 Analysis without a Peccei-Quinn mechanism $\quad 35$

6.2 Analysis with a Peccei-Quinn mechanism 37

$6.3 V_{u d}, V_{u s}$, and CKM unitarity 40

7 Conclusion $\quad 42$

A Solution of $V_{R}$ in the $P$-symmetric mLRSM 43

A.1 Region of validity 44

B Mass eigenstates of the Higgs fields $\quad 45$ 
C.1 Matching to the LEFT in the basis of ref. [1] 48

$\begin{array}{ll}\text { D Observables } & 49\end{array}$

D.1 Leptonic and semileptonic decays $\quad 49$

D.2 $\Delta B=1$ and $\Delta S=1$ processes $\quad 52$

D.2.1 $B \rightarrow J / \psi K_{S}$

D.2.2 The $B \rightarrow X_{d, s} \gamma$ branching ratio $\quad 54$

D.2.3 The $B \rightarrow X_{d, s} \gamma$ CP asymmetry $\quad 54$

D.2.4 The $B \rightarrow K^{* 0} \gamma$ CP asymmetry $\quad 55$

D.2.5 Corrections to the B meson widths $\quad 55$

D.2.6 $K_{L} \rightarrow \pi^{0} e^{+} e^{-} \quad 56$

$\begin{array}{lll}\text { E Renormalization group equations } & 58\end{array}$

\section{Introduction}

Left-right (LR) symmetric models [2-6] provide a framework for a dynamical theory of parity $(P)$ violation and led to the prediction of right-handed neutrinos and the see-saw mechanism, well before neutrino oscillations were discovered $[7,8]$. Apart from providing a natural explanation of parity violation and neutrino masses, LR models give rise to a rich phenomenology. For example, due to the see-saw mechanism, LR models violate lepton number, which leads to an interesting interplay of different contributions to neutrinoless double beta decay [7-14]. The resulting signal could very well be measurable, even in the normal hierarchy with small neutrino masses. The high-energy analogue, the so-called KeungSenjanović process [15], is a promising probe of the same source of lepton number violation at the LHC or future colliders. In addition, the presence of right-handed charged currents mediated by $W_{R}$ exchange and of heavy scalar bosons with flavor-changing interactions lead to a rich flavor phenomenology, with new contributions to a broad range of processes including CP violation in meson mixing and decays [16-22], nuclear $\beta$-decay [23, 24], electroweak precision observables [25-27], and electric dipole moments (EDMs) of leptons, nucleons, nuclei, atoms, and molecules [28-31].

Direct searches for right-handed gauge bosons at colliders constrain their masses to be larger than a few $\mathrm{TeV}$ [32-34]. To accommodate the non-observation of large flavorchanging-neutral-current processes, the new scalars associated with left-right models must have even larger masses, $\gtrsim \mathcal{O}(10) \mathrm{TeV}$. The gap between the right-handed scale, where parity is spontaneously broken, and the electroweak scale makes left-right symmetric models amenable to effective field theory (EFT) techniques. In particular, at the right-handed scale the theory can be matched onto the Standard Model EFT ${ }^{1}$ (SMEFT). Although a large number of SMEFT operators is induced, the associated Wilson coefficients only depend on

\footnotetext{
${ }^{1}$ Depending on the mass scale of right-handed neutrinos, it might be appropriate to match to the SMEFT extended with right-handed neutrinos instead [35-37]. In this work, we focus on the quark sector of left-right models and do not discuss leptonic observables in great detail.
} 
a handful of fundamental parameters. The relatively small set of parameters (compared to, for instance, supersymmetric models) allows for a global analysis of the parameter space. Several such analyses have been performed in the literature, see e.g. [26, 38-40]. For instance, recently refs. $[39,40]$ considered the correlation between direct and indirect $\mathrm{CP}$ violation in kaon decays and the neutron electric dipole moment, setting lower bounds on the $W_{R}$ mass (for earlier work including also $\Delta F=2$ transitions in $\mathrm{B}$ mesons, see e.g. refs. $[22,41])$. A large amount of work has also been devoted to the phenomenology of the leptonic sector of left-right models [10, 42-47].

In this work we investigate the minimal left-right symmetric model with a generalized $P$ symmetry. In particular, we focus on the hadronic sector of the model and leave the interesting phenomenology related to the lepton sector (from neutrinoless double beta decay to lepton flavor violation) for future work. Our aim is to perform a true global analysis of the low-energy phenomenology of the $P$-symmetric minimal left-right model in order to determine the allowed parameter space of the model, focusing mainly on a potential lower bound on the $W_{R}$ mass. As the SMEFT operators affect many processes that are used to extract the elements of the Cabibbo-Kobayashi-Maskawa (CKM) quark mixing matrix, it is not consistent to simply use the values for the quark mixing angles and phases obtained from a SM fit. We therefore extend previous analyses and refit the CKM parameters in combination with the new parameters associated with left-right models (which we denote by LR parameters). This requires us to include a large number of observables that are discussed in detail in this work. At the same time this allows us to consider possible beyond-the-Standard-Model (BSM) solutions to recent discrepancies in some of these observables, in particular the determinations of the $V_{u d}$ and $V_{u s}$ CKM elements, in a consistent manner. This analysis draws from ref. [48] which performed a similar study for one specific dimension-six SMEFT operator that is induced in left-right symmetric models.

The hadronic observables we consider depend on perturbative and non-perturbative theoretical quantities and controlling their uncertainties is crucial to obtain strong bounds on BSM physics. Advances in lattice QCD have reduced the error on decay constants and form factors entering the theoretical expressions of leptonic and semileptonic meson decays to the permille level in the case of light quarks and percent level for heavy quarks [49]. Similarly, the local matrix elements of $\Delta F=2$ operators required for $\varepsilon_{K}$ and the $B_{d, s}-\bar{B}_{d, s}$ mass splittings have uncertainties of a few percent. More recently, the first complete lattice QCD calculations of $K \rightarrow \pi \pi$ matrix elements have appeared [50], leading to a SM prediction for direct $\mathrm{CP}$ violation in kaon decays with $\sim 40 \%$ error. These calculations have also helped to reduce the error on hadronic electric dipole moments [31, 48]. In addition to the inclusion of a large number of observables, our analysis improves upon previous literature by using state-of-the-art theoretical predictions for hadronic and nuclear matrix elements and by taking advantage of recent theoretical advances like the improved SM prediction of $\varepsilon_{K}$ [51]. Our use of the SMEFT framework allows us to include QCD corrections, in particular those arising between $\mu=M_{W_{R}}$ and $\mu=m_{W}$, in a systematic way. We discuss the residual theoretical uncertainties, which mostly affect the nucleon and nuclear EDMs, $\Delta F=2$ processes dominated by long-distance contributions (such as the $K-\bar{K}$ mass difference or $D-\bar{D}$ oscillations), and hadronic $B$ meson decays. 
Although the mLRSM leads to interesting signatures at high energies [15, 41, 52, 53], here we focus on low-energy phenomenology and do not explicitly include LHC observables in our analysis. While such a combination is certainly interesting, an EFT analysis might not be appropriate for collider phenomenology, depending on the mass of BSM fields. The indirect bounds we find turn out to be sufficiently strong for most of the parameter space to ensure that direct production of right-handed gauge bosons is not yet accessible at the LHC. The combined analysis of low- and high-energy probes within the mLRSM is certainly very interesting and left to future work.

We start by introducing the LR model in section 2 . We subsequently integrate out the heavy LR fields and match onto the SMEFT in section 3, where we also discuss the renormalization group ( $R G$ ) evolution to low energies and the subsequent matching onto the $\mathrm{SU}(3)_{c} \times \mathrm{U}(1)_{\mathrm{QED}}$ invariant EFT, known as LEFT. Section 4 performs the matching onto the low-energy description of $\mathrm{QCD}$, chiral perturbation theory $(\chi \mathrm{PT})$, which is relevant for low-energy hadronic and nuclear observables. Some of the most important observables included in our analyses are described in section 5, where we also discuss the impact of the new features of our analysis for the $\Delta F=2$ observables that have been the focus of previous works $[40,41]$, while others are relegated to appendix D. We finally present our results in section 6 and conclude in section 7, while several Appendices are dedicated to technical details.

\section{Minimal left-right models}

\subsection{Particle content}

The gauge group of LR models [2-6] is given by $\mathrm{SU}(2)_{L} \times \mathrm{SU}(2)_{R} \times \mathrm{U}(1)_{B-L}$. The fermions are assigned to representations of the above gauge group as follows,

$$
\begin{aligned}
Q_{L} & =\left(\begin{array}{c}
u_{L} \\
d_{L}
\end{array}\right) \in(2,1,1 / 3), & Q_{R}=\left(\begin{array}{l}
u_{R} \\
d_{R}
\end{array}\right) \in(1,2,1 / 3), \\
L_{L} & =\left(\begin{array}{c}
\nu_{L} \\
l_{L}
\end{array}\right) \in(2,1,-1), & L_{R}=\left(\begin{array}{c}
\nu_{R} \\
l_{R}
\end{array}\right) \in(1,2,-1) .
\end{aligned}
$$

In the scalar sector, a field transforming under both $\mathrm{SU}(2)_{L}$ and $\mathrm{SU}(2)_{R}, \phi \in\left(2,2^{*}, 0\right)$, is introduced, which allows for interactions that give rise to the mass terms of the fermions after electroweak symmetry breaking (EWSB). Additional scalar fields are then used to break the LR gauge group to that of the SM. We focus on the version of the LR model, called the minimal left-right symmetric model (mLRSM), in which this is done with two triplets, $\Delta_{L, R}$, assigned to $(3,1,2)$ and $(1,3,2)$, respectively. These fields can be written as

$$
\phi=\left(\begin{array}{cc}
\phi_{1}^{0} & \phi_{2}^{+} \\
\phi_{1}^{-} & \phi_{2}^{0}
\end{array}\right), \quad \Delta_{L, R}=\left(\begin{array}{cc}
\delta_{L, R}^{+} / \sqrt{2} & \delta_{L, R}^{++} \\
\delta_{L, R}^{0} & -\delta_{L, R}^{+} / \sqrt{2}
\end{array}\right),
$$

and they transform as $\phi \rightarrow U_{L} \phi U_{R}^{\dagger}, \Delta_{L, R} \rightarrow U_{L, R} \Delta_{L, R} U_{L, R}^{\dagger}$ under $\mathrm{SU}(2)_{L, R}$ transformations. 
Having specified the particle content we can write the complete Lagrangian as follows

$$
\begin{aligned}
\mathcal{L}= & i \bar{Q}_{L} \not D Q_{L}+i \bar{Q}_{R} \not D Q_{R}+i \bar{L}_{L} \not D L_{L}+i \bar{L}_{R} \not D L_{R} \\
& -\frac{1}{4} W_{L \mu \nu}^{I} W_{L}^{I \mu \nu}-\frac{1}{4} W_{R \mu \nu}^{I} W_{R}^{I \mu \nu}-\frac{1}{4} \mathcal{B}_{\mu \nu} \mathcal{B}^{\mu \nu}-\frac{1}{4} G_{\mu \nu}^{a} G^{a \mu \nu} \\
& +\operatorname{Tr}\left[\left(D_{\mu} \phi\right)^{\dagger} D^{\mu} \phi\right]+\operatorname{Tr}\left[\left(D_{\mu} \Delta_{L}\right)^{\dagger} D^{\mu} \Delta_{L}\right]+\operatorname{Tr}\left[\left(D_{\mu} \Delta_{R}\right)^{\dagger} D^{\mu} \Delta_{R}\right]-V\left(\phi, \Delta_{L, R}\right) \\
& -\left[\bar{Q}_{L}(\Gamma \phi+\tilde{\Gamma} \tilde{\phi}) Q_{R}+\bar{L}_{L}\left(\Gamma_{l} \phi+\tilde{\Gamma}_{l} \tilde{\phi}\right) L_{R}+\bar{L}_{L}^{c} i \tau_{2} \Delta_{L} Y_{L} L_{L}+\bar{L}_{R}^{c} i \tau_{2} \Delta_{R} Y_{R} L_{R}+\text { h.c. }\right] \\
& -\theta \frac{g_{s}^{2}}{32 \pi^{2}} G_{\mu \nu}^{a} \tilde{G}^{a \mu \nu}-\theta_{R} \frac{g_{R}^{2}}{32 \pi^{2}} W_{R \mu \nu}^{I} \tilde{W}_{R}^{I \mu \nu}-\theta_{L} \frac{g_{L}^{2}}{32 \pi^{2}} W_{L \mu \nu}^{I} \tilde{W}_{L}^{I \mu \nu}-\theta_{B-L} \frac{g_{B-L}^{2}}{32 \pi^{2}} \mathcal{B}_{\mu \nu} \tilde{\mathcal{B}}^{\mu \nu},
\end{aligned}
$$

where $I$ and $a$ are $\mathrm{SU}(2)_{L, R}$ and $\mathrm{SU}(3)_{c}$ indices, $W_{L, R}^{\mu \nu}, \mathcal{B}^{\mu \nu}$, and $G^{\mu \nu}$ are the field strengths of the $\mathrm{SU}(2)_{L, R}, \mathrm{U}(1)_{B-L}$, and $\mathrm{SU}(3)_{c}$ gauge groups, while $g_{L, R}, g_{B-L}$, and $g_{s}$ are their gauge couplings. Furthermore, $\psi^{c}=C \bar{\psi}^{T}$ indicates charge conjugation and $\tilde{\phi}=\tau_{2} \phi^{*} \tau_{2}$. Finally, $\theta_{i}$ denote the $\theta$ terms for each of the different gauge groups, where $\tilde{X}^{\mu \nu}=\frac{1}{2} \epsilon^{\alpha \beta \mu \nu} X_{\alpha \beta}$ with $\epsilon^{\mu \nu \alpha \beta}$ the completely asymmetric tensor and $\epsilon^{0123}=+1$. The first three lines give the kinetic terms of the fermions, the gauge fields, and the scalars, respectively. The fourth line gives the interactions of the fermions with the scalars. The last line describes the various $\theta$ terms.

The couplings $Y_{L, R}$ are symmetric $3 \times 3$ matrices which give rise to Majorana masses for the neutrinos, while the $\Gamma_{(l)}$ and $\tilde{\Gamma}_{(l)}$ matrices are general $3 \times 3$ matrices which provide the Dirac masses of the fermions. We work in the basis where the $e_{L, R}$ and $u_{L, R}$ fields correspond to their mass eigenstates. The $d_{L, R}$ fields that reside in the quark doublets are then related to their mass eigenstates by $d_{L, R}=V_{L, R} d_{L, R}^{\text {mass }}$, where $V_{L, R}$ are the left- and right-handed CKM matrices.

Finally, the covariant derivative is given by,

$$
D_{\mu}=\partial_{\mu}-i g_{s} G_{\mu}^{a} t^{a}-i g_{L} T_{L}^{I} W_{L \mu}^{I}-i g_{R} T_{R}^{I} W_{R \mu}^{I}-i \frac{g_{B-L}}{2}(B-L) \mathcal{B}_{\mu},
$$

where $t^{a}$ and $T_{L, R}^{I}$ are the generators of $\mathrm{SU}(3)_{c}$ and $\mathrm{SU}(2)_{L, R}$ in the representation of the field that $D_{\mu}$ works on.

Together with the Higgs potential, $V\left(\phi, \Delta_{L, R}\right)$ (see e.g. ref. [54] for a detailed analysis), Eq. (2.3) specifies the complete model. However, since we will be integrating out the heavy new fields, we will need the Lagrangian in the broken phase, which requires the vacuum expectation values of the scalar fields.

\section{$2.2 \quad$ Symmetry breaking}

The breaking of the LR gauge group is realized by the vacuum expectation values (vevs) of the scalar fields

$$
\langle\phi\rangle=\sqrt{1 / 2}\left(\begin{array}{cc}
\kappa & 0 \\
0 & \kappa^{\prime} e^{i \alpha}
\end{array}\right), \quad\left\langle\Delta_{L}\right\rangle=\sqrt{1 / 2}\left(\begin{array}{cc}
0 & 0 \\
v_{L} e^{i \theta_{L}} & 0
\end{array}\right), \quad\left\langle\Delta_{R}\right\rangle=\sqrt{1 / 2}\left(\begin{array}{cc}
0 & 0 \\
v_{R} & 0
\end{array}\right),
$$

where all parameters are real after gauge transformations have been used to eliminate two of the possible phases [6]. The necessary conditions to obtain a symmetry-breaking pattern of this form have been discussed in refs. [55-58]. 
We will assume that the $\mathrm{SU}(3)_{c} \times \mathrm{SU}(2)_{L} \times \mathrm{SU}(2)_{R} \times \mathrm{U}(1)_{B-L}$ gauge group is broken down to $\mathrm{SU}(3)_{c} \times \mathrm{U}(1)_{\mathrm{QED}}$ in two steps. In the first step the vev of the right-handed triplet, $v_{R}$, breaks the $\mathrm{SU}(2)_{L} \times \mathrm{SU}(2)_{R} \times \mathrm{U}(1)_{B-L}$ gauge group down to $\mathrm{SU}(2)_{L} \times \mathrm{U}(1)_{Y}$. This vev defines the high scale of the model, and gives the main contribution to the masses of the heavy fields: the right-handed gauge bosons, the right-handed neutrinos, and the heavy Higgs fields. At the electroweak scale the vevs of the bidoublet, $\kappa$ and $\kappa^{\prime} e^{i \alpha}$, then break $\mathrm{SU}(2)_{L} \times \mathrm{U}(1)_{Y}$ to $\mathrm{U}(1)_{\mathrm{QED}}$, and are of the order of the EW scale, $\sqrt{\kappa^{2}+\kappa^{\prime 2}}=v \simeq 246 \mathrm{GeV}$. Finally, $v_{L}$ contributes to the masses of the light neutrinos through the second to last term in eq. (2.3) and one would therefore expect that $v_{L} \lesssim \mathcal{O}(1 \mathrm{eV})$.

The hierarchy between the different vevs allows us to describe the effects of the new heavy particles in an effective field theory in which the heavy fields are integrated out. This has the advantage of simplifying loop calculations and allows one to resum large logarithms. We will therefore integrate out the heavy BSM particles after the first step of symmetry breaking, i.e. after the right-handed triplet obtains its vev. We will work in the phase where the SM gauge group remains unbroken and match onto operators that are invariant under $\mathrm{SU}(2)_{L} \times \mathrm{U}(1)_{Y}$.

Before discussing this matching procedure we briefly describe the two possible discrete symmetries between left- and right-handed fields that can be implemented in LR models as well as the constraints they place on the model parameters.

\subsection{Left-right symmetries}

One of the motivations for LR models is the possibility of having a symmetry between leftand right-handed particles at high energies. Here we discuss the two possible transformations that relate left- and right-handed fields,

$$
\begin{aligned}
& P:\left\{\begin{array}{l}
Q_{L} \longleftrightarrow Q_{R}, \quad L_{L} \longleftrightarrow L_{R}, \quad \phi \longleftrightarrow \phi^{\dagger}, \quad \Delta_{L} \longleftrightarrow \Delta_{R}, \\
\tau \cdot W_{L \mu}^{I} \longleftrightarrow \tau \cdot W_{R}^{I \mu}, \quad t^{a} G_{\mu}^{a} \rightarrow t^{a} G^{a \mu}, \quad \mathcal{B}_{\mu} \rightarrow \mathcal{B}^{\mu},
\end{array}\right. \\
& C:\left\{\begin{array}{l}
Q_{L} \longleftrightarrow Q_{R}^{c}, \quad L_{L} \longleftrightarrow L_{R}^{c}, \quad \phi \longleftrightarrow \phi^{T}, \quad \Delta_{L} \longleftrightarrow \Delta_{R}^{*}, \\
\tau \cdot W_{L \mu} \longleftrightarrow\left(\tau \cdot W_{R \mu}\right)^{*}, \quad t^{a} G_{\mu}^{a} \rightarrow\left(t^{a} G_{\mu}^{a}\right)^{*}, \quad \mathcal{B}_{\mu} \rightarrow \mathcal{B}_{\mu},
\end{array}\right.
\end{aligned}
$$

where the first is related to parity and the second to charge conjugation [41].

If either of these two transformations leaves an LR model invariant we will refer to it as left-right symmetric. Given our assumptions for the vevs of the scalar fields, such a symmetry will be broken by the vev of the right-handed triplet, $v_{R}$. Nevertheless, these symmetries still provide useful constraints on the model parameters. For example, the $C$ and $P$ symmetries require the $\mathrm{SU}(2)_{L, R}$ gauge couplings to be equal, $g_{L}=g_{R}$, at the LR scale and they restrict the number of parameters that appear in the Higgs potential. In addition, they imply several relations between the couplings of the fermions to the scalars and, in the $P$-symmetric case, set the $\theta_{i}$ terms to zero. This is summarized by

$$
\begin{array}{llll}
P: & \Gamma=\Gamma^{\dagger}, & \tilde{\Gamma}=\tilde{\Gamma}^{\dagger}, & Y_{L}=Y_{R}, \quad \theta=\theta_{i}=0, \\
C: & \Gamma=\Gamma^{T}, & \tilde{\Gamma}=\tilde{\Gamma}^{T}, & Y_{L}=Y_{R}^{\dagger} .
\end{array}
$$


For our purposes, the most important consequence of the above relations is their impact on the quark mass matrices, which can be written as

$$
M_{u}=\sqrt{1 / 2} \kappa\left(\Gamma+\xi e^{-i \alpha} \tilde{\Gamma}\right), \quad M_{d}=\sqrt{1 / 2} \kappa\left(\xi e^{i \alpha} \Gamma+\tilde{\Gamma}\right),
$$

where $\xi \equiv \kappa^{\prime} / \kappa$. Given our choice of basis the up-type mass matrix is already diagonal, $M_{u}=$ $\operatorname{diag}\left(m_{u}, m_{c}, m_{t}\right)$, while the down-type mass matrix satisfies $V_{L}^{\dagger} M_{d} V_{R}=\operatorname{diag}\left(m_{d}, m_{s}, m_{b}\right)$. From eqs. (2.7) and (2.8) one can see that the mass matrices become symmetric in the $C$-symmetric case, while the $P$-symmetric matrices are hermitian in the limit $\xi \sin \alpha \rightarrow 0$.

In both cases these restrictions are enough to relate the right-handed CKM matrix to the left-handed one. In the $C$-symmetric case there is the simple relation [59]

$$
C: \quad V_{R}=K_{u} V_{L}^{*} K_{d}
$$

where $K_{u}=\operatorname{diag}\left(e^{i \theta_{u}}, e^{i \theta_{c}}, e^{i \theta_{t}}\right)$ and $K_{d}=\operatorname{diag}\left(e^{i \theta_{d}}, e^{i \theta_{s}}, e^{i \theta_{b}}\right)$ are diagonal matrices of phases, of which one combination can be set to zero, while the rest remains unconstrained. As a result, the mixing angles in both matrices will be equal.

The $P$-symmetric case is somewhat more involved. Here the right-handed CKM matrix takes a simple form only in the limit where $\xi \sin \alpha \rightarrow 0$

$$
P: \quad V_{R}=S_{u} V_{L} S_{d}, \quad(\xi \sin \alpha=0),
$$

where $S_{u, d}$ are diagonal matrices of signs, one combination of which is unphysical, such that there are 32 solutions. In the general $P$-symmetric case, the above relation is only approximately satisfied and acquires corrections $\sim \xi \sin \alpha$. These corrections can appear with ratios of the quark masses and so they are expected to be small as long as $\xi \sin \alpha \ll$ $m_{b} / m_{t}[60]$. The solution for $V_{R}$ has been derived in refs. [60,61] and expresses $V_{R}$ in terms of the quark masses, $V_{L}$, and $\xi \sin \alpha$. This implies that, although there are 32 different solutions, $V_{R}$ does not introduce any additional model parameters in this case. The approximate expressions we use in this work are described in appendix A.

Although both the $P$ - and $C$-symmetric cases are phenomenologically viable, due to the more constrained and predictive nature of right-handed CKM matrix, we will focus on the scenario with a $P$ symmetry in what follows.

\subsection{Strong CP problem and $P$ symmetry}

In the case of a $P$ symmetry the QCD $\theta$ term is explicitly forbidden, see eq. (2.7), and at scales where the parity symmetry is unbroken, we have $\theta=0$. However, after EWSB and the breaking of parity, the quark mass matrices generally obtain a phase which contributes to the physical combination $\bar{\theta} \equiv \theta+\operatorname{Arg} \operatorname{Det} M_{u} M_{d}=\operatorname{Arg} \operatorname{Det} V_{R}^{\dagger}$. This contribution is calculable [40] and to good approximation given by

$$
\bar{\theta} \simeq \frac{m_{t}}{2 m_{b}} \sin \alpha \tan 2 \beta, \quad \tan 2 \beta=\frac{2 \xi}{1-\xi^{2}} .
$$

As the $\bar{\theta}$ term is a marginal operator, this source of $\mathrm{CP}$ violation is not suppressed by any ratio of scales. Using the current neutron EDM limit, $d_{n}<1.8 \cdot 10^{-26} \mathrm{e} \mathrm{cm}[62]$ and the 
lattice-QCD result $d_{n}=-(1.5 \pm 0.7) \cdot 10^{-16} \bar{\theta}$ e $\mathrm{cm}[63]$, gives $|\bar{\theta}|<1.2 \cdot 10^{-10}$. In the absence of another mechanism to account for the QCD $\bar{\theta}$ term (for instance through a Peccei-Quinn mechanism or by allowing for explicit parity violation in the mLRSM Lagrangian), this limit implies that

$$
\sin \alpha \tan 2 \beta<5.8 \cdot 10^{-12},
$$

which effectively forces $\sin \alpha=0$, for practical purposes. Thus, the strong CP problem in the Standard Model, i.e. the smallness of $\bar{\theta}<10^{-10}$, is transferred in the $P$-symmetric mLRSM to the requirement of setting $\sin \alpha \tan 2 \beta<5.8 \cdot 10^{-12}$ by hand. Of course, in both the SM and the mLRSM these are not really problems in the sense of inconsistencies. In fact, in both models these small parameters are technically natural implying that, once chosen small, there are no large radiative corrections that renormalize the parameters. It has been argued that the strong CP problem is therefore not a problem, see e.g. ref. [64].

Nevertheless, there is something uneasy about these small numbers. Why does nature prefer absence of $\mathrm{CP}$ violation in the strong sector? There seem to be no anthropic arguments that motivate a small $\bar{\theta}[65,66]$. A popular way to dynamically remove the $\bar{\theta}$ term is through the Peccei-Quinn mechanism that leads to a new field, the axion, which can potentially be linked to Dark Matter. Of course, the Peccei-Quinn mechanism is an ad hoc addition to the mLRSM and it can be argued that it is less minimal than simply setting certain phases to be small by hand (ref. [67] discusses how infrared and ultraviolet solutions can be separated using EDM experiments).

In this work, we do not wish to choose between these two approaches and therefore perform two analyses. In the first, we describe the EDM phenomenology in the mLRSM in presence of a Peccei-Quinn mechanism. In this case, EDMs are induced by flavorconserving dimension-six operators and an interesting pattern of $\mathrm{CP}$-violating observables appears. We will see that the Peccei-Quinn mechanism releases us from the requirement that $\sin \alpha \tan 2 \beta$ must be very small. This allows for a relatively light $M_{W_{R}}$ as potentially dangerous contributions to kaonic CP violation due to the CKM phase can be cancelled against contributions proportional to $\sin \alpha$. In this case, we find a stringent lower bound on $M_{W_{R}}$ of order of a few $\mathrm{TeV}$. These conclusions agree qualitatively with refs. [39, 40]. In general the PQ mechanism in presence of additional sources of $\mathrm{CP}$ violation (beyond the $\bar{\theta}$ term) leads to CP-violating axion interactions with hadrons that can be limited by astrophysical constraints or searched for in dedicated experiments [68-71]. We do not specify the PQ mechanism and do not consider these couplings here.

We also study the pure mLRSM with $P$ symmetry where no PQ mechanism is present. As this version of the mLRSM is more constrained, due to eq. (2.12), it leads to significantly stronger limits on the mass of right-handed gauge bosons.

\section{Matching and renormalization group equations}

In this section we integrate out the heavy fields and match onto gauge invariant operators in the SMEFT [72]. In order to do so, we assume that the right-handed scalar triplet has obtained a vev, thereby breaking $\mathrm{SU}(2)_{R}$, while $\mathrm{SU}(2)_{L} \times \mathrm{U}(1)_{Y}$ remains unbroken. At this stage there are several relevant heavy fields with masses $\mathcal{O}\left(v_{R}\right)$ : 
Gauge bosons. The breaking of $\mathrm{SU}(2)_{R}$ leads to a charged and a neutral gauge boson, $W_{R}^{ \pm}$and $Z_{R}$, with $\mathcal{O}\left(v_{R}\right)$ masses, which arise from the $W_{R}^{I}$ and $\mathcal{B}$ fields. The remaining linear combinations of the gauge fields make up the $\mathrm{SM} \mathrm{SU}(2)_{L}$ and hypercharge fields. The heavy charged bosons can be written as

$$
W_{R \mu}^{ \pm}=\frac{W_{R \mu}^{1} \mp i W_{R \mu}^{2}}{\sqrt{2}}, \quad M_{W_{R}}^{2}=\frac{1}{2} g_{R}^{2} v_{R}^{2} .
$$

The neutral $W_{R}^{3}$ and $\mathcal{B}$ bosons mix and can be written in terms of mass eigenstates

$$
\begin{aligned}
\left(\begin{array}{c}
W_{R \mu}^{3} \\
\mathcal{B}_{\mu}
\end{array}\right) & =\left(\begin{array}{cc}
c_{R} & s_{R} \\
-s_{R} & c_{R}
\end{array}\right)\left(\begin{array}{c}
Z_{R \mu} \\
B_{\mu}
\end{array}\right), \quad s_{R}=\frac{g_{B-L}}{\sqrt{g_{B-L}^{2}+g_{R}^{2}}}, \quad c_{R}=\frac{g_{R}}{\sqrt{g_{B-L}^{2}+g_{R}^{2}}}, \\
M_{B}^{2} & =0,
\end{aligned}
$$

where $B_{\mu}$ is the hypercharge field of the SM. This field then couples to hypercharge, $Y=Q-T_{L}^{3}=\frac{B-L}{2}+T_{R}^{3}$, with gauge coupling $g^{\prime}=s_{R} g_{R}=c_{R} g_{B-L}$. The $W_{L}^{I}$ fields stay massless as well implying that, after integrating out the heavy gauge fields, the covariant derivative reduces to that of the SM, $D_{\mu}=\partial_{\mu}-i g_{s} G_{\mu}^{a} t^{a}-i g_{L} T_{L}^{I} W_{L \mu}^{I}-i g^{\prime} Y B_{\mu}$, where $g=g_{L}=g_{R}$.

Scalar $\mathbf{S U}(2)_{L}$ doublet. After $\Delta_{R}$ acquires a vev, the bi-doublet $\phi$ can be written in terms of two $\mathrm{SU}(2)_{L}$ doublets, $\phi=\left(\phi_{1}, \phi_{2}\right)$, of which one linear combination obtains an $\mathcal{O}\left(v_{R}\right)$ mass. The relation to the mass eigenstates is ${ }^{2}$

$$
\left(\begin{array}{c}
\tilde{\phi}_{1} \\
\phi_{2}
\end{array}\right)=\left(\begin{array}{cc}
-c_{\beta} & s_{\beta} e^{-i \alpha} \\
s_{\beta} e^{i \alpha} & c_{\beta}
\end{array}\right)\left(\begin{array}{c}
\varphi \\
\varphi_{H}
\end{array}\right), \quad M_{\varphi}^{2}=0, \quad M_{H}^{2}=\frac{\alpha_{3} v_{R}^{2}}{2} \frac{1+\xi^{2}}{1-\xi^{2}}
$$

where the mixing angles are given by $s_{\beta}=\sin \beta, c_{\beta}=\cos \beta$, and $t_{\beta}=\tan \beta=\xi$, while $\varphi_{H}$ is the heavy doublet, $\varphi$ is the SM Higgs doublet, and $\alpha_{3}$ is a parameter in the Higgs potential, in the notation of ref. [54].

In addition to the heavy states mentioned above, the right-handed neutrinos obtain an $\mathcal{O}\left(v_{R}\right)$ Majorana mass while the right-handed triplet, $\Delta_{R}$, gives rise to a heavy doublycharged and a heavy neutral scalar, $\delta_{R}^{++}$and $\operatorname{Re} \delta_{R}^{0}$, respectively. ${ }^{3}$ However, since these fields mainly couple to the leptons and scalar fields they have a limited effect on observables that probe the couplings to quarks. We therefore do not pursue the effects of the $\nu_{R}, \delta_{R}^{++}$, and $\operatorname{Re} \delta_{R}^{0}$ fields, and focus on the matching conditions that arise from integrating out the $W_{R}^{ \pm}, Z_{R}$, and $\varphi_{H}$ fields.

\footnotetext{
${ }^{2}$ The appearance of the vevs of the bi-doublet through $\xi=s_{\beta} / c_{\beta}$ in eq. (3.3) might be somewhat surprising as we are working in the unbroken phase of $\mathrm{SU}(2)_{L}$ and $\phi$ has not acquired a vev yet. In principle, Eq. (3.3) can be written in terms of the parameters in the Higgs potential and $v_{R}$ alone. However, the parameters of the Higgs potential can be eliminated in favor of $\xi$ by use of the minimum equations, see appendix B for details.

${ }^{3}$ The remaining components of $\Delta_{R}$, namely $\delta_{R}^{+}$and $\operatorname{Im} \delta_{R}^{0}$, are the would-be-Goldstone bosons that are eaten by the $W_{R}^{ \pm}$and $Z_{R}$ fields, see appendix B for more details.
} 


\subsection{Matching conditions at $\mu=M_{W_{R}}$}

To obtain the matching conditions, we integrate out the heavy fields and work up to dimension six in the EFT, i.e. we keep terms that are suppressed by up to two powers of the high scale. All the heavy fields are integrated out at a common scale which we take to be $\mu=M_{W_{R}}$. Since $\mathrm{SU}(2)_{R}$ is explicitly broken at this stage, we now move to the mass basis for the right-handed down-type quarks. This is achieved by a rotation of the right-handed downtype quarks, $d_{R} \rightarrow V_{R} d_{R}$. The relevant interactions that receive matching contributions are a right-handed charged current, $C_{H u d}$, as well as several four-quark operators ${ }^{4}$

$$
\begin{aligned}
\mathcal{L}= & \left(C_{H u d}^{i j} \tilde{\varphi}^{\dagger} i D_{\mu} \varphi \bar{u}^{i} \gamma^{\mu} d^{j}+\text { h.c. }\right) \\
& -C_{1 R R}^{i j l m} \bar{d}^{i} \gamma^{\mu} u^{j} \bar{u}^{l} \gamma_{\mu} d^{m}-C_{2 R R}^{i j l m} \bar{d}_{\alpha}^{i} \gamma^{\mu} u_{\beta}^{j} \bar{u}_{\beta}^{l} \gamma_{\mu} d_{\alpha}^{m} \\
& +C_{1, q d}^{i j l m} \bar{q}^{i} \gamma^{\mu} q^{j} \bar{d}^{l} \gamma_{\mu} d^{m}+C_{2, q d}^{i j l m} \bar{q}_{\alpha}^{i} \gamma^{\mu} q_{\beta}^{j} \bar{d}_{\beta}^{l} \gamma_{\mu} d_{\alpha}^{m} \\
& +C_{1, q u}^{i j l m} \bar{q}^{i} \gamma^{\mu} q^{j} \bar{u}^{l} \gamma_{\mu} u^{m}+C_{2, q u}^{i j l m} \bar{q}_{\alpha}^{i} \gamma^{\mu} q_{\beta}^{j} \bar{u}_{\beta}^{l} \gamma_{\mu} u_{\alpha}^{m} \\
& +\left(C_{1, q u q d}^{i j l m} \varepsilon^{a b} \bar{q}_{a}^{i} u^{j} \bar{q}_{b}^{l} d^{m}+C_{2, q u q d}^{i j l m} \varepsilon^{a b} \bar{q}_{a \alpha}^{i} u_{\beta}^{j} \bar{q}_{b \beta}^{l} d_{\alpha}^{m}+\text { h.c. }\right),
\end{aligned}
$$

where $q=\left(u_{L}, d_{L}\right)^{T}$ denotes the doublet of left-handed fields, $d=d_{R}$ and $u=u_{R}$ denote right-handed fields for up- and down-type quarks, $i, \ldots, m$ are flavor indices, and $\alpha$ and $\beta$ are color indices. The Wilson coefficients at the scale $\mu=M_{W_{R}}$ are given by

$$
\begin{aligned}
C_{H u d}^{i j} & =\frac{g_{R}^{2}}{M_{W_{R}}^{2}} \frac{\xi e^{i \alpha}}{1+\xi^{2}} V_{R, i j}, \\
C_{1 R R}^{i j l m} & =\frac{g_{R}^{2}}{2 M_{W_{R}}^{2}} V_{R, j i}^{*} V_{R, l m}, \quad C_{2 R R}^{i j l m}=0, \\
C_{1, q u}^{i j l m} & =0, \quad C_{2, q u}^{i j l m}=-\frac{1}{2} Y_{u H}^{i m}\left(Y_{u H}^{*}\right)^{j l}\left[\frac{1}{M_{H}^{2}}+\frac{g_{R}^{2}}{32 \pi^{2} M_{W_{R}}^{2}}\left(\frac{1-\xi^{2}}{1+\xi^{2}}\right)^{2}\left(\ln \frac{M_{H}^{2}}{M_{W_{R}}^{2}}-1\right)\right], \\
C_{1, q d}^{i j l m} & =0, \quad C_{2, q d}^{i j l m}=-\frac{1}{2} Y_{d H}^{i m}\left(Y_{d H}^{*}\right)^{j l}\left[\frac{1}{M_{H}^{2}}+\frac{g_{R}^{2}}{32 \pi^{2} M_{W_{R}}^{2}}\left(\frac{1-\xi^{2}}{1+\xi^{2}}\right)^{2}\left(\ln \frac{M_{H}^{2}}{M_{W_{R}}^{2}}-1\right)\right], \\
C_{1, q u q d}^{i j l m} & =\frac{1}{M_{H}^{2}} Y_{u H}^{i j} Y_{d H}^{l m}, \quad C_{2, q u q d}^{i j, l m}=0,
\end{aligned}
$$

where $Y_{u H, d H}$ are the Yukawa couplings of $\varphi_{H}$,

$$
Y_{u H}=\frac{\sqrt{2}}{v} \frac{M_{d}\left(1+\xi^{2}\right)-2 \xi e^{i \alpha} M_{u}}{1-\xi^{2}}, \quad Y_{d H}=\frac{\sqrt{2}}{v} \frac{M_{u}\left(1+\xi^{2}\right)-2 \xi e^{-i \alpha} M_{d}}{1-\xi^{2}} V_{R} .
$$

The $C_{q d, q u}$ Wilson coefficients are important as they mediate $\Delta F=2$ processes at low energies. They are generated by tree-level $\varphi_{H}$ exchange, and, at scales below $\mu=M_{W_{R}}$, by loop diagrams induced by $W_{R}$ interactions. Both types of contributions are phenomenologically relevant, as $M_{H}$ tends to be heavier than $M_{W_{R}}$. For this reason we work at tree-level for the contributions $\sim M_{H}^{-2}$, while keeping loop-level contributions

\footnotetext{
${ }^{4}$ We have chosen a basis of dimension-six operators that is most convenient for our calculations. The comparison to the standard Warsaw basis is given in appendix C.
} 
proportional to $(4 \pi)^{-2} M_{W_{R}}^{-2}$. In particular, we include corrections to $C_{q u, q d}$ in eq. (3.5) scaling as $\left(4 \pi M_{W_{R}}\right)^{-2}$ that arise from self-energy graphs for $\varphi_{H},{ }^{5}$ while dropping loop diagrams involving $\varphi_{H}$ that scale $\sim\left(4 \pi M_{H}\right)^{-2}$. The same approximation is used for $M_{H}$ in the above expressions, were we include loop contributions due to $W_{R}$ interactions that are enhanced by factors of $\left(M_{H} / M_{W_{R}}\right)^{2}$. This implies that $M_{H}$ corresponds to the one-loop expression for the physical Higgs mass up-to-and-including potentially large $\left(M_{H}^{4}\right) /\left(4 \pi M_{W_{R}}\right)^{2}$ terms, but misses loop contributions without the $M_{H}^{2} / M_{W_{R}}^{2}$ enhancement, $M_{H}^{2}=M_{H, \text { phys }}^{2}\left[1+\mathcal{O}\left((4 \pi)^{-2}\right)\right]$.

For the loop contributions to $\Delta F=2$ operators from diagrams involving $W_{L}$ and $W_{R}$ bosons, we find that they are cancelled by those in the EFT when performing the matching at $\mu=M_{W_{R}}$. The finite parts of this result in principle depend on the scheme and the treatment of evanescent operators, which appear for the four-fermion interactions and impact the way Dirac structures are reduced to our basis of operators. ${ }^{6}$ We employ $\overline{\mathrm{MS}}$ throughout our calculations, however, for the evanescent terms, we adopt a scheme in which their contributions are compensated by local counterterms [76-78]. In particular, in the evaluation of box diagrams we use the relation $\gamma_{\mu} \gamma_{\nu} P_{L} \otimes \gamma_{\nu} \gamma_{\mu} P_{R}=d P_{L} \otimes P_{R}-E_{L R}^{(2)}$ to reduce the Dirac structures we encounter, where $E_{L R}^{(2)}$ is the evanescent operator that defines our scheme. We subsequently use the following Fierz identity, $\left(\bar{\psi}_{1} \gamma_{\mu} P_{L} \psi_{2}\right)\left(\bar{\psi}_{3} \gamma^{\mu} P_{R} \psi_{4}\right)=$ $-2\left(\bar{\psi}_{1} P_{R} \psi_{4}\right)\left(\bar{\psi}_{3} P_{L} \psi_{2}\right)-E_{L R}^{(F 1)}$ to further reduce the loop contributions to our basis of operators. This scheme is equivalent to that of ref. [79], with $a_{\mathrm{ev}}=-1 / 2$.

When evolving the Lagrangian in eq. (3.4) from $M_{W_{R}}$ to the electroweak scale, the dipole operators are induced by the $C_{1,2 \text { quqd }}$ coefficients. These dipole interactions can be written in an $\mathrm{SU}(2)_{L}$-invariant form as follows

$$
\begin{aligned}
\mathcal{L}_{\text {dip }}= & -\frac{g^{\prime}}{\sqrt{2}} \bar{q} \sigma^{\mu \nu} B_{\mu \nu} \Gamma_{B}^{u} u \tilde{\varphi}-\frac{g^{\prime}}{\sqrt{2}} \bar{q} \sigma^{\mu \nu} B_{\mu \nu} \Gamma_{B}^{d} d \varphi \\
& -\frac{g}{\sqrt{2}} \bar{q} \sigma^{\mu \nu} W_{L, \mu \nu}^{I} \tau^{I} \Gamma_{W}^{u} u \tilde{\varphi}-\frac{g}{\sqrt{2}} \bar{q} \sigma^{\mu \nu} W_{L, \mu \nu}^{I} \tau^{I} \Gamma_{W}^{d} d \varphi \\
& -\frac{g_{s}}{\sqrt{2}} \bar{q} \sigma^{\mu \nu} G_{\mu \nu}^{a} t^{a} \Gamma_{g}^{u} u \tilde{\varphi}-\frac{g_{s}}{\sqrt{2}} \bar{q} \sigma^{\mu \nu} G_{\mu \nu}^{a} t^{a} \Gamma_{g}^{d} d \varphi+\text { h.c. },
\end{aligned}
$$

at low energies, the off-diagonal components of these interactions significantly contribute to $\Delta F=1$ observables, while the diagonal components give rise to EDMs. It is useful to define the following combinations of the $\Gamma_{W, B, g}^{u, d}$ couplings,

$$
\begin{aligned}
\frac{Q_{u} m_{u_{j}}}{v} C_{\gamma u}^{i j} & =-\left(\Gamma_{B}^{u}+\Gamma_{W}^{u}\right)^{i j}, & \frac{Q_{d} m_{d_{j}}}{v} C_{\gamma d}^{i j} & =-\left(V_{L}^{\dagger} \Gamma_{B}^{d}-V_{L}^{\dagger} \Gamma_{W}^{d}\right)^{i j}, \\
\frac{m_{u_{j}}}{v} C_{g u}^{i j} & =\left(\Gamma_{g}^{u}\right)^{i j}, & \frac{m_{d_{j}}}{v} C_{g d}^{i j} & =\left(V_{L}^{\dagger} \Gamma_{g}^{d}\right)^{i j}
\end{aligned}
$$

where $Q_{u}$ and $Q_{d}$ are the electric charges of the quarks and $C_{\gamma d, \gamma u}$ are the combinations that will give rise to the electromagnetic dipole moments of the quarks after electroweak

\footnotetext{
${ }^{5}$ As discussed in refs. [73-75], only the combination of these diagrams with box diagrams involving $W_{L}$ and $W_{R}$ bosons gives a gauge-invariant result.

${ }^{6}$ This scheme dependence in the matching is removed when computing physical matrix elements in the EFT.
} 
symmetry breaking, while $C_{g d, g u}$ are the gluonic dipole moments. We introduced a CKM factor in the couplings for the down-type operators in anticipation of a later rotation to the mass basis.

\subsection{Renormalization group equations below $M_{W_{R}}$}

The evolution of the effective Lagrangian from $\mu=M_{W_{R}}$ to the electroweak scale requires the renormalization group equations (RGEs). For the four-quark operators these take the form $[80,81]$

$$
\frac{d}{d \ln \mu} \vec{C}^{i j l m}=\left(\begin{array}{cccc}
\frac{\alpha_{s}}{4 \pi} \gamma_{R R} & 0 & 0 & 0 \\
\frac{1}{(4 \pi)^{2}} \gamma_{E W}^{D} & \frac{\alpha_{s}}{4 \pi} \gamma_{L R} & 0 & 0 \\
\frac{1}{(4 \pi)^{2}} \gamma_{E W}^{U} & 0 & \frac{\alpha_{s}}{4 \pi} \gamma_{L R} & 0 \\
0 & 0 & 0 & \frac{\alpha_{s}}{4 \pi} \gamma_{L R L R}
\end{array}\right)_{a b c d}^{i j l m} \cdot \vec{C}^{a b c d}
$$

where $\vec{C}^{T}=\left(C_{1 R R}, C_{2 R R}, C_{1, q d}, C_{2, q d}, C_{1, q u}, C_{2, q u}, C_{1, q u q d}, C_{2, q u q d}\right)$. The diagonal terms describe one-loop QCD corrections. The $\gamma_{R R}$ and $\gamma_{L R}$ terms are diagonal in generation indices

$$
\gamma_{R R}=\delta_{a i} \delta_{b j} \delta_{c l} \delta_{d m}\left(\begin{array}{cc}
-6 / N_{c} & 6 \\
6 & -6 / N_{c}
\end{array}\right), \quad \gamma_{L R}=\delta_{a i} \delta_{b j} \delta_{c l} \delta_{d m}\left(\begin{array}{cc}
6 / N_{c} & 0 \\
-6 & -6 \frac{N_{c}^{2}-1}{N_{c}}
\end{array}\right)
$$

where $N_{c}=3$ is the number of colors. For the operators with $(\bar{L} R)(\bar{L} R)$ chiralities the anomalous dimensions are

$$
\gamma_{L R L R}=\left(\begin{array}{cc}
2 / N_{c}-6 N_{c} & -4 \\
4 & 2 / N_{c}+2 N_{c}
\end{array}\right) \delta_{a i} \delta_{b j} \delta_{c l} \delta_{d m}+\left(\begin{array}{cc}
-8 & 8 / N_{c}-4 N_{c} \\
8 / N_{c} & -4
\end{array}\right) \delta_{c i} \delta_{b j} \delta_{a l} \delta_{d m} .
$$

The operators, $C_{i R R}$, contribute to $C_{i q d}$ through electroweak loops captured by $\gamma_{E W}^{U, D}$

$$
\gamma_{E W}^{D}=\frac{2}{v^{2}} \delta_{a l} \delta_{d m}\left(M_{u}^{\dagger}\right)_{b j}\left(M_{u}\right)_{i c}\left(\begin{array}{ll}
0 & 1 \\
1 & 0
\end{array}\right), \quad \gamma_{E W}^{U}=\frac{2}{v^{2}} \delta_{c l} \delta_{b m}\left(V_{R}^{\dagger} M_{d}^{\dagger}\right)_{d j}\left(M_{d} V_{R}\right)_{i a}\left(\begin{array}{ll}
0 & 1 \\
1 & 0
\end{array}\right)
$$

The dipole operators are induced through the following RGEs [82-84]

$$
\begin{aligned}
& \frac{d}{d \ln \mu}\left(C_{\gamma u}^{i j}, C_{g u}^{i j}\right)^{T}=\frac{\alpha_{s}}{4 \pi} \gamma_{\text {dip }} \cdot\left(\begin{array}{c}
C_{\gamma u}^{i j} \\
C_{g u}^{i j}
\end{array}\right)+\frac{1}{(4 \pi)^{2}} \sum_{k \in d, s, b} \frac{\left(V_{R}^{\dagger} M_{d}^{\dagger}\right)_{l k}}{m_{u_{j}}} \gamma_{q u q d}^{u} \cdot\left(\begin{array}{c}
C_{1 q u q d}^{k j i l} \\
C_{2 q u q d}^{k j i k}
\end{array}\right), \\
& \frac{d}{d \ln \mu}\left(C_{\gamma d}^{i j}, C_{g d}^{i j}\right)^{T}=\frac{\alpha_{s}}{4 \pi} \gamma_{\text {dip }} \cdot\left(\begin{array}{c}
C_{\gamma d}^{i j} \\
C_{g d}^{i j}
\end{array}\right)+\frac{1}{(4 \pi)^{2}} \sum_{k \in u, c} \frac{m_{u_{k}}}{m_{d_{j}}} \gamma_{q u q d}^{d} \cdot\left(\begin{array}{c}
V_{L, i l} C_{1 q u q d}^{l k k j} \\
V_{L, i l} C_{2 q u q d}^{l k k j}
\end{array}\right),
\end{aligned}
$$

where

$$
\gamma_{\text {dip }}=\left(\begin{array}{cc}
8 C_{F} & -8 C_{F} \\
0 & 16 C_{F}-4 N_{c}
\end{array}\right), \quad \gamma_{q u q d}^{d}=\left(\begin{array}{cc}
2 \frac{Q_{u}}{Q_{d}} & 2 N_{c} \frac{Q_{u}}{Q_{d}} \\
-2 & -4 C_{F}
\end{array}\right),
$$

where $C_{F}=\frac{N_{c}^{2}-1}{2 N_{c}}$ and $\gamma_{q u q d}^{u}$ can be obtained from $\gamma_{q u q d}^{d}$ by $Q_{u} \leftrightarrow Q_{d}$.

Finally, the $C_{H u d}$ operator does not evolve under QCD. 


\subsection{Matching at $\mu=m_{W}$}

After evolving the effective operators in eq. (3.4) to the electroweak scale we integrate out the top quark as well as the Higgs, $W$, and $Z$ bosons. Because $\mathrm{SU}(2)_{L}$ has now been broken, we move to the mass basis of the left-handed down-type quarks. This can be achieved by the following flavor rotation, $d_{L} \rightarrow V_{L} d_{L}$, so that the left-handed quark doublet becomes, $q=\left(u_{L}, V_{L} d_{L}\right)^{T}$. The relevant four-fermion operators below the electroweak scale can be written as

$$
\begin{aligned}
\mathcal{L}= & -C_{1 L L}^{i j l m} \bar{d}_{L}^{i} \gamma^{\mu} u_{L}^{j} \bar{u}_{L}^{l} \gamma_{\mu} d_{L}^{m}-C_{2 L L}^{i j l m} \bar{d}_{L \alpha}^{i} \gamma^{\mu} u_{L \beta}^{j} \bar{u}_{L \beta}^{l} \gamma_{\mu} d_{L \alpha}^{m} \\
& -\left(C_{1 L R}^{i j l m} \bar{d}_{L}^{i} \gamma^{\mu} u_{L}^{j} \bar{u}_{R}^{l} \gamma_{\mu} d_{R}^{m}+C_{2 L R}^{i j l m} \bar{d}_{L \alpha}^{i} \gamma^{\mu} u_{L \beta}^{j} \bar{u}_{R \beta}^{l} \gamma_{\mu} d_{R \alpha}^{m}+\text { h.c. }\right) \\
& -C_{1 R R}^{i j l m} \bar{d}_{R}^{i} \gamma^{\mu} u_{R}^{j} \bar{u}_{R}^{l} \gamma_{\mu} d_{R}^{m}-C_{2 R R}^{i j l m} \bar{d}_{R \alpha}^{i} \gamma^{\mu} u_{R \beta}^{j} \bar{u}_{R \beta}^{l} \gamma_{\mu} d_{R \alpha}^{m} \\
& +C_{4}^{i j l m} \bar{d}_{L}^{i} \gamma^{\mu} d_{L}^{j} \bar{d}_{R}^{l} \gamma^{\mu} d_{R}^{m}+C_{5}^{i j l m} \bar{d}_{L \alpha}^{i} \gamma^{\mu} d_{L \beta}^{j} \bar{d}_{R \beta}^{l} \gamma^{\mu} d_{R \alpha}^{m} \\
& +\left(C_{1, q u q d}^{i j l m} \varepsilon^{a b} \bar{q}_{a}^{i} u_{R}^{j} \bar{q}_{b}^{l} d_{R}^{m}+C_{2, q u q d}^{i j l m} \varepsilon^{a b} \bar{q}_{a \alpha}^{i} u_{R \beta}^{j} \bar{q}_{b \beta}^{l} d_{R \alpha}^{m}+\text { h.c. }\right) \\
& +C_{\tilde{G}} \frac{g_{s}}{6} f_{a b c} \epsilon^{\alpha \beta \mu \nu} G_{\alpha \beta}^{a} G_{\mu \rho}^{b} G_{\nu}^{c \rho} .
\end{aligned}
$$

Most of the above operators have a similar form to the $\mathrm{SU}(2)_{L}$-invariant ones in eq. (3.4), apart from those in the first, second, and last lines. Those in the first two lines are additional four-quark operators, generated by the $\mathrm{SM}$ and $C_{H u d}$, while the last line describes the so-called Weinberg operator, which is induced through one-loop diagrams.

The dipole operators take the following form below the electroweak scale

$$
\begin{aligned}
\mathcal{L}_{\text {dip }}= & -\frac{e Q_{u}}{2} \sum_{i, j \in u, c} m_{u_{j}} C_{\gamma u}^{i j} \bar{q}_{L}^{i} \sigma^{\mu \nu} F_{\mu \nu} q_{R}^{j}-\frac{e Q_{d}}{2} \sum_{i, j \in d, s, b} m_{d_{j}} C_{\gamma d}^{i j} \bar{q}_{L}^{i} \sigma^{\mu \nu} F_{\mu \nu} q_{R}^{j} \\
& -\frac{g_{s}}{2} \sum_{i, j \in u, c} m_{u_{j}} C_{g u}^{i j} \bar{q}_{L}^{i} \sigma^{\mu \nu} G_{\mu \nu}^{a} t^{a} q_{R}^{j}-\frac{g_{s}}{2} \sum_{i, j \in d, s, b} m_{d_{j}} C_{g d}^{i j} \bar{q}_{L}^{i} \sigma^{\mu \nu} G_{\mu \nu}^{a} t^{a} q_{R}^{j}+\text { h.c. }
\end{aligned}
$$

The tree-level matching leads to

$$
\begin{array}{ll}
C_{1 L L}^{i j l m}=2 \sqrt{2} G_{F}\left(V_{L}^{\dagger}\right)^{i j}\left(V_{L}\right)^{l m}, & C_{2 L L}=0, \\
C_{1 L R}^{i j l m}=\left(V_{L}^{\dagger}\right)^{i j}\left(C_{H u d}\right)^{l m}, & C_{2 L R}=0,
\end{array}
$$

while the coefficients of the remaining four-quark operators, $C_{i R R}$ and $C_{i q u q d}$, are unaffected at the $W_{L}$ threshold. $C_{4}$ and $C_{5}$ get a tree-level contribution from $C_{q d}$, as well as a contribution from loop diagrams involving $C_{1,2 R R}^{i j l m}$ and $W_{L}$ exchange

$$
\begin{aligned}
C_{4}^{i j l m}\left(m_{W}\right)= & V_{L a i}^{*} V_{L b j} C_{1, q d}^{a b l m}\left(m_{W}\right) \\
& +\frac{g_{L}^{2}}{4(4 \pi)^{2}} C_{2 R R}^{l t t m} V_{L t i}^{*} V_{L t j} x_{t}\left(\ln m_{W}^{2} / \mu^{2}+\frac{3}{1-x_{t}}+\frac{\left(4+\left(x_{t}-2\right) x_{t}\right) \ln x_{t}}{\left(1-x_{t}\right)^{2}}\right) \\
& +\frac{g_{L}^{2}}{4(4 \pi)^{2}}\left(C_{2 R R}^{l c t m} V_{L t i}^{*} V_{L c j}+C_{2 R R}^{l t c m} V_{L c i}^{*} V_{L t j}\right) \\
& \times \sqrt{x_{c} x_{t}} \frac{\left(\ln m_{W}^{2} / \mu^{2}-1\right)\left(x_{t}-1\right)+\left(x_{t}-4\right) \ln x_{t}}{x_{t}-1} \\
& +\frac{g_{L}^{2}}{4(4 \pi)^{2}} C_{2 R R}^{l c c m} V_{L c i}^{*} V_{L c j} x_{c}\left(1-3 \ln m_{W}^{2} / \mu^{2}\right)
\end{aligned}
$$


where $x_{i}=m_{i}^{2} / m_{W}^{2}$. The first, second and third contributions result from diagrams involving an internal $t-t, t-c$, and $c-c$ pair, respectively, and we dealt with the appearance of evanescent operators as described above eq. (3.7). A similar equation holds for $C_{5}$, with the replacements, $C_{2 R R} \rightarrow C_{1 R R}$ and $C_{1 q d} \rightarrow C_{2 q d}$.

Finally, one-loop contributions to the Weinberg operator [85, 86] and dipole moments [87] appear

$$
\begin{aligned}
C_{\gamma u}^{i j}\left(m_{W}^{-}\right)= & C_{\gamma u}^{i j}\left(m_{W}^{+}\right)+\frac{2}{(4 \pi)^{2}} \sum_{k=d, s, b} \frac{m_{d_{k}}}{m_{u_{j}} Q_{u}} V_{L}^{i k} C_{H u d}^{* j k}, \\
C_{g u}^{i j}\left(m_{W}^{-}\right)= & C_{g u}^{i j}\left(m_{W}^{+}\right), \\
C_{\gamma d}^{i j}\left(m_{W}^{-}\right)= & C_{\gamma d}^{i j}\left(m_{W}^{+}\right)+\frac{2}{(4 \pi)^{2}} \sum_{k=u, c} \frac{m_{u_{k}}}{m_{d_{j}} Q_{d}} V_{L}^{* k i} C_{H u d}^{k j} \\
& +\frac{1}{(4 \pi)^{2}} \frac{m_{t}}{m_{d_{j}} Q_{d}} V_{L}^{* t i} C_{H u d}^{t j}\left[Q_{u} f_{W}\left(x_{t}\right)+g_{W}\left(x_{t}\right)\right], \\
C_{g d}^{i j}\left(m_{W}^{-}\right)= & C_{g d}^{i j}\left(m_{W}^{+}\right)-\frac{1}{(4 \pi)^{2}} \frac{m_{t}}{m_{d_{j}}} V_{L}^{* t i} C_{H u d}^{t j} f_{W}\left(x_{t}\right), \\
C_{\tilde{G}}\left(m_{W}^{-}\right)= & C_{\tilde{G}}\left(m_{W}^{+}\right)-\frac{\alpha_{s}}{8 \pi} \operatorname{Im} C_{g u}^{(t t)},
\end{aligned}
$$

where $x_{t}=m_{t}^{2} / m_{W}^{2}$ and

$$
f_{W}(x)=\frac{x^{3}+3 x-4-6 x \ln x}{2(x-1)^{3}}, \quad g_{W}(x)=\frac{4+x(x-11)}{2(x-1)^{2}}+3 \frac{x^{2} \ln x}{(x-1)^{3}} .
$$

\subsection{Renormalization group equations below $\mu=m_{W}$}

Below $\mu=m_{W}$, the QCD running for the relevant four-quark operators is equivalent to the running above the electroweak scale; the $C_{1 L L}$ and $C_{2 L L}$ coefficients follow the same RGEs as $C_{1 R R}$ and $C_{2} R R$, while the RGEs of $C_{1 L R}$ and $C_{2 L R}$ (and $C_{4}$ and $C_{5}$ ) correspond to those of $C_{1 q d}$ and $C_{2 q d}$. The running of $C_{1,2 q u q d}$ and $C_{1,2 R R}$ is unchanged below $\mu=m_{W}$.

Instead, the mixing of the $C_{i} R R$ operators with $C_{4,5}$ operators changes from eq. (3.9) to

$$
\begin{aligned}
\frac{d C_{4}^{a b c d}}{d \ln \mu} & =\frac{1}{4 \pi^{2}} m_{u_{i}} m_{u_{j}}\left[C_{1 L L}^{a i j b} C_{2 R R}^{c j i d}+C_{2 L L}^{a i j b} C_{1 R R}^{c j i d}+N_{c} C_{2 L L}^{a i j b} C_{2 R R}^{c j i d}\right], \\
\frac{d C_{5}^{a b c d}}{d \ln \mu} & =\frac{1}{4 \pi^{2}} m_{u_{i}} m_{u_{j}} C_{1 L L}^{a i j b} C_{1 R R}^{c j i d} .
\end{aligned}
$$

The RGEs for the flavor-diagonal dipole operators must be extended to include the Weinberg operator. The QCD part of the RGEs becomes

$$
\frac{d}{d \ln \mu} \vec{C}_{\text {dip }}=\frac{\alpha_{s}}{4 \pi} \gamma_{\text {dip }}^{\prime} \vec{C}_{\text {dip }},
$$

with $\vec{C}_{\text {dip }}=\left(\operatorname{Im} C_{\gamma q}^{(q q)}, \operatorname{Im} C_{g q}^{(q q)}, C_{\tilde{G}}\right)^{T}$, and $[85,88,89]$

$$
\gamma_{\text {dip }}^{\prime}=\left(\begin{array}{ccc}
8 C_{F} & -8 C_{F} & 0 \\
0 & 16 C_{F}-4 N_{c} & 2 N_{c} \\
0 & 0 & N_{c}+2 n_{f}+\beta_{0}
\end{array}\right),
$$


where $\beta_{0}=\left(11 N_{c}-2 n_{f}\right) / 3$, with $n_{f}$ the number of active flavors. The $C_{1,2 L R}$ coefficients also contribute to dipole operators, which is captured by

$$
\begin{aligned}
\frac{d}{d \ln \mu}\left(C_{\gamma u}^{i j}, C_{g u}^{i j}\right)^{T} & =\frac{\alpha_{s}}{4 \pi} \sum_{k \in d, s, b} \frac{m_{d_{k}}}{m_{u_{j}}} \gamma_{L R}^{u} \cdot\left(\begin{array}{c}
C_{1 L R}^{j k i k} \\
C_{2 L R}^{j k i k}
\end{array}\right)^{*}+\ldots, \\
\frac{d}{d \ln \mu}\left(C_{\gamma d}^{i j}, C_{g d}^{i j}\right)^{T} & =\frac{\alpha_{s}}{4 \pi} \sum_{k \in u, c} \frac{m_{u_{k}}}{m_{d_{j}}} \gamma_{L R}^{d} \cdot\left(\begin{array}{c}
C_{1 L R}^{k j k i} \\
C_{2 L R}^{k j k i}
\end{array}\right)+\ldots,
\end{aligned}
$$

where the dots stand for the additional terms on the right-hand side of eq. (3.12), and [87]

$$
\gamma_{L R}^{d}=\frac{1}{(4 \pi)^{2}}\left(\begin{array}{cc}
32 \frac{Q_{u}}{Q_{d}}+\frac{64}{3} & 160 \frac{Q_{u}}{Q_{d}} \\
-\frac{16}{3} & 8
\end{array}\right) .
$$

$\gamma_{L R}^{u}$ can be obtained from $\gamma_{L R}^{d}$ by $Q_{u} \leftrightarrow Q_{d}$.

\subsection{Matching contributions below $\mu=m_{W}$}

Below the electroweak scale we integrate out the bottom and charm quarks at the respective mass scales. At the bottom threshold this gives rise to matching contributions to the Weinberg operator and the dipole moments of the up-type quarks

$$
\begin{aligned}
C_{\gamma u}^{i j}\left(m_{b}^{-}\right) & =C_{\gamma u}^{i j}\left(m_{b}^{+}\right)+\frac{1}{8 \pi^{2}} \frac{Q_{d} m_{b}}{Q_{u} m_{u_{j}}}\left[C_{1 L R}^{b i j b}+N_{c} C_{2 L R}^{b i j b}\right]^{*}, \\
C_{g u}^{i j}\left(m_{b}^{-}\right) & =C_{g u}^{i j}\left(m_{b}^{+}\right)-\frac{1}{8 \pi^{2}} \frac{m_{b}}{m_{u_{j}}}\left[C_{1 L R}^{b i j b}\right]^{*} \\
C_{\tilde{G}}\left(m_{b}^{-}\right) & =C_{\tilde{G}}\left(m_{b}^{+}\right)-\frac{\alpha_{s}}{8 \pi} \operatorname{Im} C_{g d}^{(b b)} .
\end{aligned}
$$

Similarly, at the charm threshold we obtain the following contributions

$$
\begin{aligned}
C_{\gamma d}^{i j}\left(m_{c}^{-}\right) & =C_{\gamma d}^{i j}\left(m_{c}^{+}\right)+\frac{1}{8 \pi^{2}} \frac{Q_{u} m_{c}}{Q_{d} m_{d_{j}}}\left[C_{1 L R}^{i c c j}+N_{c} C_{2 L R}^{i c c j}\right], \\
C_{g d}^{i j}\left(m_{c}^{-}\right) & =C_{g d}^{i j}\left(m_{c}^{+}\right)-\frac{1}{8 \pi^{2}} \frac{m_{c}}{m_{d_{j}}} C_{1 L R}^{i c c j}, \\
C_{\tilde{G}}\left(m_{c}^{-}\right) & =C_{\tilde{G}}\left(m_{c}^{+}\right)-\frac{\alpha_{s}}{8 \pi} \operatorname{Im} C_{g u}^{(c c)} .
\end{aligned}
$$

Finally, at $\mu=m_{c}$ we find the following matching contributions to the $C_{4,5}$ coefficients

$$
\begin{aligned}
C_{4}^{i j l m}\left(m_{c}\right)= & C_{4}\left(m_{c}^{+}\right)+\frac{m_{c}^{2}}{(4 \pi)^{2}}\left(1+2 \ln m_{W}^{2} / \mu^{2}+2 \ln x_{c}\right) \\
& \times\left[C_{1 L L}^{i c c j} C_{2 R R}^{l c c m}+C_{2 L L}^{i c c j} C_{1 R R}^{l c c m}+N_{c} C_{2 L L}^{i c c j} C_{2 R R}^{l c c m}\right], \\
C_{5}^{i j l m}\left(m_{c}\right)= & C_{5}\left(m_{c}^{+}\right)+\frac{m_{c}^{2}}{(4 \pi)^{2}}\left(1+2 \ln m_{W}^{2} / \mu^{2}+2 \ln x_{c}\right) C_{1 L L}^{i c c j} C_{1 R R}^{l c c m} .
\end{aligned}
$$

At low energies the $C_{4,5}$ coefficients mediate $\Delta F=2$ processes. Working at fixed, one-loop order and collecting the matching contributions at $\mu=M_{H}, M_{W_{R}}, m_{W}$ and $m_{c}$, as well as the electroweak running contributions in between these thresholds, we reproduce the 
expressions in ref. [22], up to terms $\sim \mathcal{O}\left(\frac{1}{(4 \pi)^{2} M_{H}^{2}}\right)$ that we neglect as explained below eq. (3.6).

In our analysis we include QCD corrections by solving the RGEs of the four-quark operators thereby evolving their Wilson coefficients from one threshold to the next. Formally, our approach is then accurate up to leading-log precision. I.e. it takes into account terms of order $\frac{1}{(4 \pi)^{2}} \ln \times\left(\frac{\alpha_{s}}{4 \pi}\right)^{n} \ln ^{n}$, but does not include all of those at order $\frac{1}{(4 \pi)^{2}} \ln \times\left(\frac{\alpha_{s}}{4 \pi}\right)^{n} \ln ^{n-1}$. Some of these terms are included in our matching equations, e.g. through the non-log terms in eqs. (3.17) and (3.27), but we neglected contributions at the same order that would arise from two-loop matching at the different thresholds. In the same way we include the leading-log contributions to the dipole operators, $C_{\gamma q}$ and $C_{g q}$.

This strategy is similar to the one followed in refs. $[22,41]$ for the contributions to $C_{4,5}$ mediated by $t-t$ graphs, but differs somewhat for those with intermediate $c-t$ or $c-c$ quarks. For the latter, ref. [22] employed the approach outlined in refs. [75, 90], which is not guaranteed to reproduce a leading-log approximation. We discuss the impact of these differences when considering $\Delta F=2$ observables in section 5.3.

\subsection{Summary}

Using the matching conditions in sections 3.1, 3.3 and 3.5, and the RGEs in sections 3.2 and 3.4, we can finally give approximate expressions for the LEFT coefficients at the scales relevant to low-energy observables. Assuming the initial scale $\mu_{0}$ is $\mu_{0}=10 \mathrm{TeV}$, we obtain the following numerical values for the charged-current four-quark operators at $\mu_{\text {low }}=2 \mathrm{GeV}$,

$$
\begin{aligned}
\frac{v^{2}}{2} C_{1 L L}^{i j k l}\left(\mu_{\text {low }}\right) & =1.15 V_{L j i}^{*} V_{L k l}, & & \frac{v^{2}}{2} C_{2 L L}^{i j k l}\left(\mu_{\text {low }}\right)=-0.34 V_{L j i}^{*} V_{L k l}, \\
\frac{v_{R}^{2}}{2} C_{1 L R}^{i j k l}\left(\mu_{\text {low }}\right) & =0.90 \frac{\xi e^{i \alpha}}{1+\xi^{2}} V_{L j i}^{*}\left(V_{R}\right)_{k l}, & \frac{v_{R}^{2}}{2} C_{2 L R}^{i j k l}\left(\mu_{\text {low }}\right) & =0.45 \frac{\xi e^{i \alpha}}{1+\xi^{2}} V_{L j i}^{*}\left(V_{R}\right)_{k l}, \\
v_{R}^{2} C_{1 R R}^{i j k l}\left(\mu_{\text {low }}\right) & =1.36\left(V_{R}\right)_{j i}^{*}\left(V_{R}\right)_{k l}, & v_{R}^{2} C_{2 R R}^{i j k l}\left(\mu_{\text {low }}\right) & =-0.65\left(V_{R}\right)_{j i}^{*}\left(V_{R}\right)_{k l},
\end{aligned}
$$

while, for the scalar operators,

$$
\begin{aligned}
& C_{1, q u q d}^{i j k l}\left(\mu_{\mathrm{low}}\right)=4.9 \frac{Y_{d H}^{k l} Y_{u H}^{i j}}{M_{H}^{2}}+2.6 \frac{Y_{d H}^{i l} Y_{u H}^{k j}}{M_{H}^{2}}, \\
& C_{2 q u q d}^{i j k l}\left(\mu_{\mathrm{low}}\right)=-0.95 \frac{Y_{d H}^{k l} Y_{u H}^{i j}}{M_{H}^{2}}-0.82 \frac{Y_{d H}^{i l} Y_{u H}^{k j}}{M_{H}^{2}} .
\end{aligned}
$$

The operators $C_{4}$ and $C_{5}$, which contribute to meson-antimeson oscillations, receive a tree level contribution from the exchange of heavy Higgses, and a loop contribution from diagrams with a $W_{R}$ and $W_{L}$ exchange. At $\mu_{\text {low }}=2 \mathrm{GeV}$, we find

$$
\begin{aligned}
& C_{4}^{i j k l}\left(\mu_{\mathrm{low}}\right)=\frac{g_{R}^{2}}{M_{W_{R}}^{2}} \sum_{a, b} a_{a b}^{(4)} \frac{m_{u_{a}} m_{u_{b}}}{m_{t}^{2}} V_{L a i}^{*} V_{L b j}\left(V_{R}\right)_{b k}^{*}\left(V_{R}\right)_{a l}, \\
& C_{5}^{i j k l}\left(\mu_{\mathrm{low}}\right)=-2.01 \frac{1}{M_{H}^{2}}\left(Y_{d H}\right)_{j k}^{*} Y_{d H}^{i l}+\frac{g_{R}^{2}}{M_{W_{R}}^{2}} \sum_{a, b} a_{a b}^{(5)} \frac{m_{u_{a}} m_{u_{b}}}{m_{t}^{2}} V_{L a i}^{*} V_{L b j}\left(V_{R}\right)_{b k}^{*}\left(V_{R}\right)_{a l},
\end{aligned}
$$


with $Y_{u H}$ and $Y_{d H}$ defined in eq. (3.6) and evaluated at $\mu=\mu_{0}$. The RG effects are captured by the $a^{(4,5)}$ coefficients, which are given by

$$
a^{(4)}=\left(\begin{array}{ccc}
0.028 & 0.028 & 0.001 \\
0.028 & 0.032 & 0.001 \\
0.001 & 0.001 & 0.00077
\end{array}\right), \quad a^{(5)}=-\left(\begin{array}{ccc}
0.16 & 0.16 & 0.034 \\
0.16 & 0.17 & 0.037 \\
0.034 & 0.037 & 0.030
\end{array}\right) \text {. }
$$

These results depend mildly on the scale $\Lambda$, and in our analysis we set $\mu_{0}=M_{W_{R}}$. If we turn off the running between $M_{W_{R}}$ and $m_{t}$ and integrate out the $W_{R}$ and heavy Higgses at the scale $\mu_{0}=m_{t}$, the values of $C_{1 R R}$ and $C_{2} R R$ are reduced (in absolute value) by about $15 \%$ and $40 \%$, respectively, while $C_{i L L}$ and $C_{i L R}$ are not affected. Similarly, the prefactors of the product of Yukawa couplings $\left.Y_{d H}^{k l} Y_{u H}^{i j}\right|_{\mu=10 \mathrm{TeV}}$ and $\left.Y_{d H}^{i l} Y_{u H}^{k j}\right|_{\mu=10 \mathrm{TeV}}$ in $C_{1 \text { quqd }}$ decrease by $\sim 10 \%$ and $\sim 30 \%$, respectively, while they decrease by $\sim 30 \%$ for both terms in $C_{2 \text { quqd. }}$. These fairly mild corrections due to the RGEs are in part due to the $\mu_{0}$ dependence of the Yukawa couplings, $Y_{q H}$, which partially compensate for the effects of the $\gamma_{L R}$ anomalous dimensions. Finally, using $\mu_{0}=m_{t}$, the upper-left $2 \times 2$ block of the $a^{(4,5)}$ coefficients in eq. (3.31) decrease by $\sim 30 \%$, while the remaining components decrease by significant factors ranging from $\sim 1 / 5$ to $\sim 1 / 40$. We collect semi-analytical results for the $\mu_{0}$ dependence of these Wilson coefficients in appendix E.

\section{The CP-violating chiral Lagrangian}

In this section we discuss the low-energy chiral Lagrangian induced by CP-violating operators involving light quarks. The construction of this Lagrangian is relevant for the study of electric dipole moments and long-distance effects in $\varepsilon_{K}$. Although the effects in EDMs and $\varepsilon_{K}$ of certain operators can be directly evaluated using lattice-QCD or QCD sum rules, there are several operators for which it is useful to employ Chiral Perturbation Theory $(\chi \mathrm{PT})$. In particular, the contributions of the LR operators in eq. (3.14) to EDMs have not been computed directly. In this case, chiral symmetry allows us to relate their contributions to CP-odd pion-nucleon couplings to matrix elements that have been computed for $K \rightarrow \pi \pi$ processes. The obtained pion-nucleon couplings can be used to estimate the leading contributions of these operators to diamagnetic atomic EDMs. In addition, deriving the mesonic Lagrangian in $\chi \mathrm{PT}$ allows us to estimate long-distance corrections to $K-\bar{K}$ mixing arising from two insertions of $\Delta S=1$ operators.

Our starting point is the following Lagrangian at the scale of a few $\mathrm{GeV}$

$$
\begin{aligned}
\mathcal{L}= & \mathcal{L}_{m_{q}=0}^{\mathrm{QCD}}-\bar{q} \mathcal{M} q-\bar{\theta} \frac{g_{s}^{2}}{64 \pi^{2}} \varepsilon^{\mu \nu \alpha \beta} G_{\mu \nu}^{a} G_{\alpha \beta}^{a}-\frac{g_{s}}{2} \bar{q}\left(i \sigma^{\mu \nu} \gamma_{5}\right) \tilde{d}_{C E} t^{a} q G_{\mu \nu}^{a} \\
& -C_{1 L R}^{A B} \bar{q} \gamma^{\mu} t^{A} P_{L} q \bar{q} \gamma_{\mu} t^{B} P_{R} q-C_{2 L R}^{A B} \bar{q}_{\alpha} \gamma^{\mu} t^{A} P_{L} q_{\beta} \bar{q}_{\alpha} \gamma_{\mu} t^{B} P_{R} q_{\beta} \\
& -\left[C_{1 L L}^{s u u d} \bar{s} \gamma^{\mu} P_{L} u \bar{u} \gamma_{\mu} P_{L} d+C_{2 L L}^{s u u d} \bar{s}_{\alpha} \gamma^{\mu} P_{L} u_{\beta} \bar{u}_{\alpha} \gamma_{\mu} P_{L} d_{\beta}+(L \leftrightarrow R)+\text { h.c. }\right],
\end{aligned}
$$

where $q$ denotes a vector of light quark fields $q=(u, d, s)^{T}, t^{a}\left(t^{A, B}\right)$ are the Gellman matrices in color (flavor) space, normalized such that $\operatorname{Tr}\left(t_{a} t_{b}\right)=\delta_{a b} / 2$, and $\mathcal{M}$ is the real 
quark mass matrix, $\mathcal{M}=\operatorname{diag}\left(m_{u}, m_{d}, m_{s}\right)$. The couplings are given by

$$
\begin{aligned}
C_{i L R}^{A B}= & \left(\delta^{A 1}-i \delta^{A 2}\right)\left(\delta^{B 1}+i \delta^{B 2}\right) C_{i L R}^{\text {duud }}+\left(\delta^{A 4}-i \delta^{A 5}\right)\left(\delta^{B 4}+i \delta^{B 5}\right) C_{i L R}^{\text {suus }} \\
& +\left(\delta^{A 4}-i \delta^{A 5}\right)\left(\delta^{B 1}+i \delta^{B 2}\right) C_{i L R}^{\text {suud }}+\left(\delta^{A 1}-i \delta^{A 2}\right)\left(\delta^{B 4}+i \delta^{B 5}\right) C_{i L R}^{\text {duus }} \\
& -\left(\delta^{A 6}-i \delta^{A 7}\right)\left(\delta^{B 6}+i \delta^{B 7}\right) C_{3+i}^{\text {sdds }}+\text { h.c. }
\end{aligned}
$$

We work in a basis where the overall phase of the mass matrix has been rotated into the $\tilde{G} G$ term to form the physical combination $\bar{\theta}$. The third term in the first line of eq. (4.1) denotes the CP-odd quark chromo-electric dipole moment with $\tilde{d}_{C E}=\operatorname{diag}\left(\tilde{d}_{u}, \tilde{d}_{d}, \tilde{d}_{s}\right)$, where $\tilde{d}_{u}=m_{u} \operatorname{Im} C_{g u}^{u u}$ and $\tilde{d}_{i}=m_{i} \operatorname{Im} C_{g d}^{i i}$ for $i=\{d, s\}$. The last two lines denote various $\mathrm{CP}$-odd four-quark operators introduced in previous sections. To obtain the above Lagrangian we have used the relation $\left(C_{4,5}^{s d d}\right)^{*}=C_{4,5}^{d s} s d$.

Our main goal will be to estimate the CP-odd pion-nucleon couplings that are induced by the LR operators and to discuss the long-distance contributions to $\bar{K}-K$ mixing generated by two insertions of the $\Delta S=1$ four-fermion terms. Compared to the Lagrangians in eqs. (3.14) and (3.15), we have omitted contributions from $C_{1, q u q d}^{i j l m}$ and $C_{2, q u q d}^{i j l m}$ as the operators involving light quarks are suppressed by small Yukawa couplings and $M_{H}^{-2}$, so that their contributions can be safely neglected. We also omitted the Weinberg operator and the quark EDMs here as we will use lattice QCD and QCD sum-rule calculations to directly obtain their contributions to EDMs in section 5.4. Finally, eq. (3.14) involves $\Delta S=1$ interactions $\sim C_{4,5}$ with $s d d d, d d s d, d s s s$, and $s s d s$ flavor structures. Unlike the $C_{i L R}^{A B}$ coefficients, which transform like $\mathbf{8}_{L} \times \mathbf{8}_{R}$ under chiral symmetry, the $C_{4,5}$ coefficients with $\Delta S=1$ have different chiral symmetry properties and we neglect them in the following as these are only generated at loop level or are suppressed by factors of small Yukawa couplings and $M_{H}^{-2}$.

\subsection{Vacuum alignment and the Peccei-Quinn mechanism}

For the purpose of chiral perturbation theory it is useful to perform several field redefinitions of the quark fields to remove meson tadpoles (tadpoles describe the disappearance of neutral Goldstone bosons to the vacuum). We start by applying a global anomalous axial U(1) transformation of the form

$$
q \rightarrow e^{i \theta_{A} \gamma^{5}} q, \quad \theta_{A}=\frac{\bar{\theta}}{2 n_{f}},
$$

with $n_{f}=3$ the number of active quark flavors, to eliminate the gluonic $\tilde{G} G$ term from the Lagrangian. The price to pay is that the quark mass matrix becomes complex. In a first step, we can ignore the shifts in the higher-dimensional qCEDMs and four-quark operators as the induced terms scale as $\bar{\theta} / \Lambda^{2}$, where $\Lambda^{2}$ collectively denotes the masses of BSM fields such as the right-handed scalar and/or gauge bosons. However, terms proportional to $\bar{\theta} / \Lambda^{2}$ do play an important role when we discuss the Peccei-Quinn mechanism below. After the rotation, the quark mass term becomes

$$
\begin{aligned}
\mathcal{L}_{m} & =-\bar{q} \mathcal{M} q+\bar{q} i \gamma^{5}\left[-\frac{2}{3}\left(2 \bar{m}+m_{s}\right)+(4 \varepsilon \bar{m}) t_{3}-\frac{4}{\sqrt{3}}\left(\bar{m}-m_{s}\right) t_{8}\right] \theta_{A} q \\
& \equiv-\bar{q} \mathcal{M} q+\bar{q} i \gamma^{5}\left[\theta_{0}+\theta_{3} t_{3}+\theta_{8} t_{8}\right] q,
\end{aligned}
$$


where we introduced $\bar{m}=\left(m_{u}+m_{d}\right) / 2$ and $2 \bar{m} \varepsilon=m_{d}-m_{u}$. The terms involving $\bar{q} i \gamma^{5} t_{3,8} q$ lead to so-called tadpole operators that allow for neutral Goldstone bosons (in this case $\pi^{0}$ and $\eta$ ) to disappear in the vacuum. In the limit of no dimension-six interactions, it is straightforward to eliminate the tadpole-inducing terms (a procedure called vacuum alignment) by performing two additional non-anomalous axial SU(3) rotations

$$
q \rightarrow e^{i\left(\alpha_{3} t_{3}+\alpha_{8} t_{8}\right) \gamma^{5}} q .
$$

By setting

$$
\alpha_{3}=\frac{\varepsilon m_{s}}{2 m_{s}+\bar{m}\left(1-\varepsilon^{2}\right)} \bar{\theta}, \quad \alpha_{8}=\frac{1}{\sqrt{3}} \frac{m_{s}-\bar{m}\left(1-\varepsilon^{2}\right)}{2 m_{s}+\bar{m}\left(1-\varepsilon^{2}\right)} \bar{\theta},
$$

the $\bar{q} i \gamma^{5} t_{3,8} q$ terms are removed and the dimension-four part of the Lagrangian becomes

$$
\mathcal{L}=-\bar{q} \mathcal{M} q-m_{*} \bar{\theta} \bar{q} i \gamma^{5} q
$$

in terms of the reduced quark mass

$$
m_{*}=\left(\frac{1}{m_{u}}+\frac{1}{m_{d}}+\frac{1}{m_{s}}\right)^{-1}=\frac{\bar{m}\left(1-\varepsilon^{2}\right)}{2}\left(1+\frac{\bar{m}\left(1-\varepsilon^{2}\right)}{2 m_{s}}\right)^{-1} .
$$

This is the usual result that shows that the theta term decouples if one of the quarks is massless. Keeping terms to $\mathcal{O}\left(\bar{\theta}^{2}\right)$ shows that the three chiral rotations proportional to $\theta_{A}$, $\alpha_{3}$, and $\alpha_{8}$ generate a term

$$
\mathcal{L}_{P Q}=\frac{1}{6} \bar{\theta}^{2} m_{*} \bar{q} q
$$

which induces a hadronic contribution to the vacuum energy. The Peccei-Quinn mechanism becomes apparent if we promote $\bar{\theta}$ to include a dynamical axion field ${ }^{7} \bar{\theta} \rightarrow \bar{\theta}+a / f_{a}$ where $a$ is the axion field and $f_{a}$ the axion decay constant. Because the vacuum energy scales as $\left(\bar{\theta}+a / f_{a}\right)^{2}$, the axion potential is minimized for $\langle a\rangle=-f_{a} \bar{\theta}$ eliminating the CP-violating term from the Lagrangian.

The story is similar, but somewhat more tedious to work out, in the presence of the dimension-six operators. With just the dimension-four terms, the entire argument could be made at the quark level with minimal reference to hadronic operators. Once the dimensionsix operators are included, it is convenient to refer to the hadronic Lagrangian explicitly. It is useful to construct the terms in the chiral Lagrangian that can induce tadpoles after the first field transformation that eliminates the gluonic $\bar{\theta}$ term. The relevant terms are given by

$$
\begin{aligned}
\mathcal{L}_{\mathrm{GB}}= & \frac{F_{0}^{2}}{4}\left(\operatorname{Tr}\left[U^{\dagger} \chi+U \chi^{\dagger}\right]+\operatorname{Tr}\left[U^{\dagger} \tilde{\chi}+U \tilde{\chi}^{\dagger}\right]\right)+\frac{F_{0}^{4}}{4} \operatorname{Tr}\left(U^{\dagger} t^{B} U t^{A}\right) \sum_{i=1,2} \mathcal{A}_{i L R} C_{i L R}^{A B} \\
& +\frac{F_{0}^{4}}{4}\left\{\sum_{i=1,2} \operatorname{Tr}\left(t^{A} \partial_{\mu} U^{\dagger} \partial^{\mu} U\right) \mathcal{A}_{i L L}^{(8)}\left[C_{i L L}^{\text {duus }}\left(\delta_{A 6}+i \delta_{A 7}\right)+\text { h.c. }\right]+\left(\begin{array}{c}
L \rightarrow R \\
U \leftrightarrow U^{\dagger}
\end{array}\right)\right\},
\end{aligned}
$$

\footnotetext{
${ }^{7}$ The performed field redefinitions become field dependent and lead to derivative axion-quark interactions. Since we do not consider axions explicitly in this paper, we do not further study these terms.
} 
where $U$ is the matrix of the pseudo-Nambu-Goldstone (pNG) boson fields

$$
U=u(\pi)^{2}=\exp \left(\frac{2 i \pi}{F_{0}}\right), \quad \pi=\frac{1}{\sqrt{2}}\left(\begin{array}{ccc}
\frac{\pi_{3}}{\sqrt{2}}+\frac{\pi_{8}}{\sqrt{6}} & \pi^{+} & K^{+} \\
\pi^{-} & -\frac{\pi_{3}}{\sqrt{2}}+\frac{\pi_{8}}{\sqrt{6}} & K^{0} \\
K^{-} & \bar{K}^{0} & -\frac{2}{\sqrt{6}} \pi_{8}
\end{array}\right),
$$

and

$$
\chi=2 B\left[\mathcal{M}+i\left(\theta_{0}+\theta_{3} t_{3}+\theta_{8} t_{8}\right)\right], \quad \tilde{\chi}=-2 i \tilde{B}\left(\tilde{d}_{0}+\tilde{d}_{3} t_{3}+\tilde{d}_{8} t_{8}\right),
$$

where we introduced the combinations $\tilde{d}_{0}=\left(\tilde{d}_{u}+\tilde{d}_{d}+\tilde{d}_{s}\right) / 3, \tilde{d}_{3}=\left(\tilde{d}_{u}-\tilde{d}_{d}\right)$, and $\tilde{d}_{8}=$ $\left(\tilde{d}_{u}+\tilde{d}_{d}-2 \tilde{d}_{s}\right) / \sqrt{3}$. Under $\mathrm{SU}(3)_{L} \times \mathrm{SU}(3)_{R}$ transformations we have $U \rightarrow R U L^{\dagger}$ such that the quark-level Lagrangian and its chiral analogue are formally invariant if the spurions $\chi$ and $\tilde{\chi}$ transform in the same way as $U$. The LR four-quark operators transform as $\mathbf{8}_{L} \times \mathbf{8}_{R}$, so that the $\mathcal{A}_{i L R}$ part of the Lagrangian is invariant if the flavor structures transform as $t^{A} \rightarrow L t^{A} L^{\dagger}$ and $t^{B} \rightarrow R t^{B} R^{\dagger}$. For the LL and RR operators, we only take into account the pieces transforming as $\mathbf{8}_{L, R} \times \mathbf{1}_{R, L}$, as the long-distance contributions of the $\mathbf{2 7}_{L, R} \times \mathbf{1}_{R, L}$ terms are suppressed by the $\Delta I=1 / 2$ rule.

The mesonic interactions are associated with 6 low-energy constants (LECs), $B, \tilde{B}$, $\mathcal{A}_{\{1,2\} L R}$, and $\mathcal{A}_{\{1,2\} L L}=\mathcal{A}_{\{1,2\} R R}$. The first is well known and relates the masses of pseudo-Goldstone bosons to the chiral condensate, while $\tilde{B}$ and $\mathcal{A}_{\{1,2\} L R}$ are related to the condensates of the higher-dimensional operators

$$
\begin{aligned}
B & =-\frac{1}{3} \frac{\langle 0|\bar{q} q| 0\rangle}{F_{0}^{2}}, & \tilde{B} & =-\frac{1}{3} \frac{\left\langle 0\left|\bar{q} g_{s} \sigma_{\mu \nu} G^{\mu \nu} q\right| 0\right\rangle}{2 F_{0}^{2}} . \\
\delta^{A B} \frac{\mathcal{A}_{1 L R}}{8} & =-\frac{\left\langle 0\left|\bar{q}_{\alpha} \gamma^{\mu} t^{A} P_{L} q_{\alpha} \bar{q}_{\beta} \gamma_{\mu} t^{B} P_{R} q_{\beta}\right| 0\right\rangle}{F_{0}^{4}}, & \delta^{A B} \frac{\mathcal{A}_{2 L R}}{8} & =-\frac{\left\langle 0\left|\bar{q}_{\alpha} \gamma^{\mu} t^{A} P_{L} q_{\beta} \bar{q}_{\beta} \gamma_{\mu} t^{B} P_{R} q_{\alpha}\right| 0\right\rangle}{F_{0}^{4}} .
\end{aligned}
$$

whereas the condensates of the LL and RR operators vanish at leading order. The LEC $B$ can also be expressed as $2 B=m_{\pi}^{2} / \bar{m}$. Using the above Lagrangian, the LECs of the four-quark operators can be determined from matrix elements of the form $\left\langle(\pi \pi)_{I=0,2}\left|O_{i}\right| K^{0}\right\rangle$ which have been computed on the lattice [50, 91, 92]. Using chiral symmetry, the same LECs can be related to matrix elements that play a role in neutrinoless double beta decay [93] or to the bag factors appearing in $K-\bar{K}$ oscillations [49], up to SU(3) corrections [94]. This leads to the following relations at leading order ${ }^{8}$

$$
\begin{aligned}
& \mathcal{A}_{1 L R}(3 \mathrm{GeV})=\frac{\mathcal{A}_{(8,8)}^{\prime}}{3 \sqrt{2} F_{0}} \simeq 2.2(1) \mathrm{GeV}^{2}, \quad \mathcal{A}_{2 L R}(3 \mathrm{GeV})=\frac{\mathcal{A}_{(8,8) \mathrm{mix}}^{\prime}}{3 \sqrt{2} F_{0}} \simeq 10.1(6) \mathrm{GeV}^{2}, \\
& \mathcal{A}_{1 L L}^{(8)}(4 \mathrm{GeV})=\mathcal{A}_{1 R R}^{(8)}(4 \mathrm{GeV})=-\frac{\mathcal{M}_{2}}{\sqrt{6} F_{0}\left(m_{K}^{2}-m_{\pi}^{2}\right)} \simeq-2.8(3) \\
& \mathcal{A}_{2 L L}^{(8)}(4 \mathrm{GeV})=\mathcal{A}_{2 R R}^{(8)}(4 \mathrm{GeV})=-\frac{\mathcal{M}_{1}}{\sqrt{6} F_{0}\left(m_{K}^{2}-m_{\pi}^{2}\right)} \simeq 1.8(3),
\end{aligned}
$$

\footnotetext{
${ }^{8}$ These relations assume that the $\mathbf{2 7}_{L, R} \times \mathbf{1}_{R, L}$ parts of the LL and RR operators provide negligible contributions to the $\left\langle(\pi \pi)_{I=0}\left|O_{i}\right| K^{0}\right\rangle$ matrix elements. These contributions can be obtained by using the LEC of the $\mathbf{2} \mathbf{7}_{L, R} \times \mathbf{1}_{R, L}$ representations, $\mathcal{A}_{(27,1)}^{\prime}$, discussed in section 5.2. Such an estimate shows that the dominant contributions to $\mathcal{M}_{1,2}$ indeed arise from the $\mathbf{8}_{L, R} \times \mathbf{1}_{R, L}$ parts of the operators.
} 
where $\mathcal{M}_{1,2}$ and $\mathcal{A}_{(8,8)(\text { mix) }}^{\prime}$ are related to matrix elements $\sim\left\langle(\pi \pi)_{I=0,2}\left|O_{i}\right| K^{0}\right\rangle$, which were determined in refs. [50, 91, 92].

The Lagrangian in eq. (4.10) leads to tadpoles as can be seen by expanding out the various terms. Introducing the ratios $\tilde{r}=\tilde{B} / B$ and $r_{i}=\left(F_{0}^{2} / B\right) \mathcal{A}_{i L R}$, the tadpole Lagrangian becomes

$$
\begin{aligned}
\mathcal{L}_{\text {tadpole }}=F_{0} B\left\{\pi^{0}\right. & {\left[\theta_{3}-\tilde{r} \tilde{d}_{3}+\frac{1}{2} \sum_{i=1,2} r_{i}\left(2 \operatorname{Im} C_{i L R}^{\text {duud }}+\operatorname{Im} C_{i L R}^{s u u s}+\operatorname{Im} C_{3+i}^{s d d s}\right)\right] } \\
& +\eta\left[\theta_{8}-\tilde{r} \tilde{d}_{8}+\frac{\sqrt{3}}{2} \sum_{i=1,2} r_{i}\left(\operatorname{Im} C_{i L R}^{\text {suus }}-\operatorname{Im} C_{3+i}^{s d d s}\right)\right] \\
& -\frac{\bar{K}^{0}+K^{0}}{\sqrt{2}} \frac{1}{2} \sum_{i=1,2} r_{i}\left(\operatorname{Im} C_{i L R}^{\text {suud }}+\operatorname{Im} C_{i L R}^{\text {duus }}\right) \\
& \left.-\frac{i\left(\bar{K}^{0}-K^{0}\right)}{\sqrt{2}} \frac{1}{2} \sum_{i=1,2} r_{i}\left(\operatorname{Re} C_{i L R}^{\text {suud }}-\operatorname{Re} C_{i L R}^{d u u s}\right)\right\}
\end{aligned}
$$

It is in principle possible to eliminate these leading tadpoles by a suitable redefinition of Goldstone fields at the hadronic level. Such a rotation, however, requires a corresponding complicated field redefinition of baryon fields, see refs. [95-97] for details. The baryon transformation was omitted in ref. [98] and led to erroneous conclusions as was also pointed out in ref. [39]. In this work, we follow ref. [31] and only perform field transformations at the quark level. We reconstruct the chiral Lagrangian after each quark transformation. This leads to the same conclusions as ref. [95] (and thus in disagreement with ref. [98] and the $m_{s} /\left(m_{u}+m_{d}\right)$ enhancement found there).

We begin by performing four axial chiral rotations on eq. (4.1), now including $\alpha_{6} t_{6}$ and $\alpha_{7} t_{7}$ rotations to remove the $K^{0}$ tadpole terms, resulting in the Lagrangian $\mathcal{L}^{\prime}$. We then construct the hadronic Lagrangian in eq. (4.10), that now depends explicitly on $\alpha_{3,6,7,8}$, and solve for $\alpha_{3,6,7,8}$ by demanding that the $\pi^{0}, K^{0}$, and $\eta$ tadpoles vanish. The solutions are given by

$$
\begin{aligned}
& \alpha_{3}=\frac{-\varepsilon m_{s}}{2 m_{s}+\bar{m}\left(1-\varepsilon^{2}\right)}\left\{-\bar{\theta}+\frac{\tilde{r}}{2 m_{s}}\left[\frac{\bar{m}+2 m_{s}}{\varepsilon \bar{m}} \tilde{d}_{3}+\sqrt{3} \tilde{d}_{8}\right]-\sum_{i=1,2} \frac{r_{i}}{2 \varepsilon \bar{m} m_{s}}\right. \\
& \left.\times\left[\left(\bar{m}+2 m_{s}\right) \operatorname{Im} C_{i L R}^{\text {duud }}+\frac{2 m_{s}+\bar{m}(1+3 \varepsilon)}{2} \operatorname{Im} C_{i L R}^{s u u s}+\frac{2 m_{s}+\bar{m}(1-3 \varepsilon)}{2} \operatorname{Im} C_{3+i}^{s d d s}\right]\right\}, \\
& \alpha_{6}=-\frac{1}{2\left(m_{d}+m_{s}\right)} \sum_{i=1,2} r_{i}\left(\operatorname{Im} C_{i L R}^{\text {duus }}+\operatorname{Im} C_{i L R}^{\text {suud }}\right) \text {, } \\
& \alpha_{7}=-\frac{1}{2\left(m_{d}+m_{s}\right)} \sum_{i=1,2} r_{i}\left(\operatorname{Re} C_{i L R}^{\text {duus }}-\operatorname{Re} C_{i L R}^{\text {suud }}\right) \text {, } \\
& \alpha_{8}=\frac{-1}{\sqrt{3}} \frac{1}{2 m_{s}+\bar{m}\left(1-\varepsilon^{2}\right)}\left\{-\left[m_{s}-\bar{m}\left(1-\varepsilon^{2}\right)\right] \bar{\theta}+\frac{3 \varepsilon \tilde{r}}{2}\left[\tilde{d}_{3}+\frac{\sqrt{3}}{\varepsilon} \tilde{d}_{8}\right]\right. \\
& \left.-\sum_{i=1,2} \frac{3 \varepsilon r_{i}}{2}\left[\operatorname{Im} C_{i L R}^{\text {duud }}+\frac{3+\varepsilon}{2 \varepsilon} \operatorname{Im} C_{i L R}^{\text {suus }}-\frac{3-\varepsilon}{2 \varepsilon} \operatorname{Im} C_{3+i}^{\text {sdds }}\right]\right\} .
\end{aligned}
$$


After these rotations the Lagrangian can be written in the following form

$$
\begin{aligned}
\mathcal{L}= & \mathcal{L}_{m_{q}=0}^{\mathrm{QCD}}-\bar{q} \mathcal{M} q+\bar{q}\left[-m_{*}\left(\bar{\theta}-\bar{\theta}_{\text {ind }}\right)+r \tilde{d}_{0}+\theta_{3}^{\prime} t_{3}+\theta_{6}^{\prime} t_{6}+\theta_{7}^{\prime} t_{7}+\theta_{8}^{\prime} t_{8}\right] i \gamma_{5} q \\
& -\frac{g_{s}}{2} \bar{q}\left(i \sigma^{\mu \nu} \gamma_{5}\right) \tilde{d}_{C E} t^{a} q G_{\mu \nu}^{a} \\
& -C_{1 L R}^{A B} \bar{q} \gamma^{\mu} t^{A} P_{L} q \bar{q} \gamma_{\mu} t^{B} P_{R} q-C_{2 L R}^{A B} \bar{q}_{\alpha} \gamma^{\mu} t^{A} P_{L} q_{\beta} \bar{q}_{\alpha} \gamma_{\mu} t^{B} P_{R} q_{\beta} \\
& -\left[C_{1 L L}^{\text {suud }} \bar{s} \gamma^{\mu} P_{L} u \bar{u} \gamma_{\mu} P_{L} d+C_{2 L L}^{\text {suud }} \bar{s}_{\alpha} \gamma^{\mu} P_{L} u_{\beta} \bar{u}_{\alpha} \gamma_{\mu} P_{L} d_{\beta}+(L \leftrightarrow R)+\text { h.c. }\right]+\ldots
\end{aligned}
$$

where the dots denote terms of dimension-eight or higher or terms proportional to $\bar{\theta}^{2}$ or $\bar{\theta} / \Lambda^{2}$. $\theta_{\text {ind }}, \theta_{3}^{\prime}, \theta_{6}^{\prime}, \theta_{7}^{\prime}$, and $\theta_{8}^{\prime}$ depend on hadronic LECs

$$
\begin{aligned}
\bar{\theta}_{\text {ind }}= & \tilde{r}\left(\frac{\tilde{d}_{u}}{m_{u}}+\frac{\tilde{d}_{d}}{m_{d}}+\frac{\tilde{d}_{s}}{m_{s}}\right) \\
& -2 \sum_{i=1,2} r_{i} \operatorname{Im}\left(\frac{m_{d}-m_{u}}{4 m_{u} m_{d}} C_{i L R}^{d u u d}+\frac{m_{s}-m_{u}}{4 m_{u} m_{s}} C_{i L R}^{s u u s}-\frac{m_{s}-m_{d}}{4 m_{d} m_{s}} \operatorname{Im} C_{3+i}^{s d d s}\right) \\
\theta_{3}^{\prime}= & \tilde{r} \tilde{d}_{3}-\sum_{i=1,2} r_{i} \operatorname{Im}\left(C_{i L R}^{\text {duud }}+\frac{1}{2} C_{i L R}^{s u u s}+\frac{1}{2} \operatorname{Im} C_{3+i}^{s d d s}\right) \\
\theta_{6}^{\prime}= & \frac{1}{2} \sum_{i=1,2} r_{i} \operatorname{Im}\left(C_{i L R}^{\text {suud }}+C_{i L R}^{\text {duus }}\right) \\
\theta_{7}^{\prime}= & -\frac{1}{2} \sum_{i=1,2} r_{i} \operatorname{Re}\left(C_{i L R}^{\text {suud }}-C_{i L R}^{\text {du } u s}\right) \\
\theta_{8}^{\prime}= & \tilde{r}_{\tilde{d}_{8}}-\frac{\sqrt{3}}{2} \sum_{i=1,2} r_{i}\left(\operatorname{Im} C_{i L R}^{\text {suus }}-\operatorname{Im} C_{3+i}^{s d d s}\right)
\end{aligned}
$$

The term $\bar{\theta}_{\text {ind }}$ is introduced because $\bar{\theta}$ effectively relaxes to $\bar{\theta}_{\text {ind }}$ if a Peccei-Quinn mechanism is applied. The expression for $\bar{\theta}_{\text {ind }}$ can be obtained by calculating the induced vacuum energy of eq. (4.17) supplemented by terms of $\mathcal{O}\left(\bar{\theta}^{2}\right)$ and $\mathcal{O}\left(\bar{\theta} / \Lambda^{2}\right)$. The latter depend linearly on $\bar{\theta}$ and ensure that, after a Peccei-Quinn mechanism is implemented through $\bar{\theta} \rightarrow \bar{\theta}_{a}=\bar{\theta}+a / f_{a}$, the minimum of the axion potential is shifted away from zero. This leads to a nonzero vev for the axion field and an effective theta angle (but suppressed by $\left.1 / \Lambda^{2}\right),\left\langle\bar{\theta}_{a}\right\rangle=\theta_{\text {ind }}$, even after implementation of the Peccei-Quinn mechanism. Once the Peccei-Quinn mechanism is applied the final Lagrangian becomes

$$
\begin{aligned}
\mathcal{L}= & \mathcal{L}_{m_{q}=0}^{\mathrm{QCD}}-\bar{q} \mathcal{M} q+\bar{q}\left[r \tilde{d}_{0}+\theta_{3}^{\prime} t_{3}+\theta_{6}^{\prime} t_{6}+\theta_{7}^{\prime} t_{7}+\theta_{8}^{\prime} t_{8}\right] i \gamma_{5} q-\frac{g_{s}}{2} \bar{q}\left(i \sigma^{\mu \nu} \gamma_{5}\right) \tilde{d}_{C E} t^{a} q G_{\mu \nu}^{a} \\
& -C_{1 L R}^{A B} \bar{q} \gamma^{\mu} t^{A} P_{L} q \bar{q} \gamma_{\mu} t^{B} P_{R} q-C_{2 L R}^{A B} \bar{q}_{\alpha} \gamma^{\mu} t^{A} P_{L} q_{\beta} \bar{q}_{\alpha} \gamma_{\mu} t^{B} P_{R} q_{\beta} \\
& -\left[C_{1 L L}^{\text {suud }} \bar{s} \gamma^{\mu} P_{L} u \bar{u} \gamma_{\mu} P_{L} d+C_{2 L L}^{\text {suud }} \bar{s}_{\alpha} \gamma^{\mu} P_{L} u_{\beta} \bar{u}_{\alpha} \gamma_{\mu} P_{L} d_{\beta}+(L \leftrightarrow R)+\text { h.c. }\right]+\ldots
\end{aligned}
$$

It can be verified explicitly that with $\theta_{3}^{\prime}, \theta_{6}^{\prime}, \theta_{7}^{\prime}$, and $\theta_{8}^{\prime}$ given by eq. (4.18), the hadronic Lagrangian in eq. (4.10) does not induce tadpoles.

After eliminating the leading tadpoles in this way, one can use eq. (4.10) to derive the low-energy effects of the CP-odd operators. The first long-distance contributions to $\bar{K}-K$ 
mixing are induced by diagrams involving two insertions of $\Delta S=1$ operators, the result of which we discuss in section 5.3.2. Instead, the most important flavor-conserving CPV interactions arise from the baryonic Lagrangian which we discuss below.

\subsection{CP-odd pion-nucleon interactions}

The relevant CPV pion-nucleon interactions arise from

$$
\begin{aligned}
\mathcal{L}_{\pi N}= & b_{0} \operatorname{Tr}(\bar{B} B) \operatorname{Tr} \chi_{+}+b_{D} \operatorname{Tr}\left(\bar{B}\left\{\chi_{+}, B\right\}\right)+b_{F} \operatorname{Tr}\left(\bar{B}\left[\chi_{+}, B\right]\right) \\
& +\tilde{b}_{0} \operatorname{Tr}(\bar{B} B) \operatorname{Tr} \tilde{\chi}_{+}+\tilde{b}_{D} \operatorname{Tr}\left(\bar{B}\left\{\tilde{\chi}_{+}, B\right\}\right)+\tilde{b}_{F} \operatorname{Tr}\left(\bar{B}\left[\tilde{\chi}_{+}, B\right]\right) \\
& +\mathcal{L}_{L R},
\end{aligned}
$$

where $b_{0, D, F}$ are LECs that can be obtained from fits to the baryon masses, $\tilde{b}_{0, D, F}$ are LECs related to the dipole operators and currently unknown, and $B$ denotes the octet baryon field

$$
B=\left(\begin{array}{ccc}
\frac{1}{\sqrt{2}} \Sigma^{0}+\frac{1}{\sqrt{6}} \Lambda & \Sigma^{+} & p \\
\Sigma^{-} & -\frac{1}{\sqrt{2}} \Sigma^{0}+\frac{1}{\sqrt{6}} \Lambda & n \\
\Xi^{-} & \Xi^{0} & -\frac{2}{\sqrt{6}} \Lambda
\end{array}\right) .
$$

We have defined

$$
\chi_{+}=u^{\dagger} \chi u^{\dagger}+u \chi^{\dagger} u, \quad \tilde{\chi}_{+}=u^{\dagger} \tilde{\chi} u^{\dagger}+u \tilde{\chi}^{\dagger} u,
$$

where $\chi$ is now given by $\chi=2 B\left[\mathcal{M}+i\left(\theta_{3}^{\prime} t_{3}+\theta_{6}^{\prime} t_{6}+\theta_{7}^{\prime} t_{7}+\theta_{8}^{\prime} t_{8}\right)\right]$. Finally, $\mathcal{L}_{L R}$ gives rise to so-called "direct" contributions to CPV meson-baryon interactions,

$$
\begin{aligned}
& \mathcal{L}_{L R}=b_{\mathbf{1}} \operatorname{Tr}(\bar{B} B) l_{1}^{a b b a}+\left\{\left[(\bar{B} B)_{j i}-\frac{\delta_{j i}}{3} \operatorname{Tr}(\bar{B} B)\right]\left[b_{\mathbf{8}}^{(1)} l_{1}^{i a a j}+b_{\mathbf{8}}^{(2)} l_{1}^{a j i a}\right]+\left(\begin{array}{c}
B \leftrightarrow \bar{B} \\
b_{\mathbf{8}}^{(1,2)} \rightarrow b_{\mathbf{8}}^{(3,4)}
\end{array}\right)\right\} \\
& +b_{\mathbf{1 0}}^{ \pm} l_{1}^{i j k l}\left[\frac{\bar{B}_{j i} B_{l k} \pm \bar{B}_{j k} B_{l i}}{2}-\frac{\delta_{i l}}{6}(\bar{B} B)_{j k}-\frac{\delta_{k j}}{6}(B \bar{B})_{l i} \mp(j \leftrightarrow l)\right] \\
& +b_{\mathbf{2 7}} l_{1}^{i j k l}\left[\frac{\bar{B}_{j i} B_{l k}+\bar{B}_{j k} B_{l i}+\bar{B}_{l i} B_{j k}+\bar{B}_{l k} B_{j i}}{4}-\frac{\delta_{i l}}{12}\{\bar{B}, B\}_{j k}-\frac{\delta_{k j}}{12}\{B, \bar{B}\}_{l i}+\frac{\delta_{j k} \delta_{i l}}{18} \operatorname{Tr}(\bar{B} B)\right] \\
& +\left(\begin{array}{c}
l_{1}^{i j k l} \leftrightarrow l_{2}^{i j k l} \\
b_{\mathbf{r}} \rightarrow \bar{b}_{\mathbf{r}}
\end{array}\right),
\end{aligned}
$$

where $l_{1,2}^{i j k l}=C_{1,2 L R}^{A B}\left(u t^{A} u^{\dagger}\right)_{i j}\left(u^{\dagger} t^{B} u\right)_{k l}$, while $b_{\mathbf{r}}$ and $\bar{b}_{\mathbf{r}}$ denote currently unknown LECs. We focus on the pion-nucleon couplings

$$
\mathcal{L}_{\pi N} \supset-\frac{\bar{g}_{0}}{2 F_{\pi}} \bar{N} \boldsymbol{\pi} \cdot \boldsymbol{\tau} N-\frac{\bar{g}_{1}}{2 F_{\pi}} \pi_{0} \bar{N} N
$$

The four-quark operators enter in the above through $\chi_{+}$, see eq. (4.12), and $\mathcal{L}_{L R}$, where the latter involves additional LECs that are currently unknown. In this work, we focus on the "indirect" contributions that we do control and neglect the terms $\sim b_{\mathbf{r}}$ and $\bar{b}_{\mathbf{r}}$. The direct pieces are expected to arise at the same order as the indirect pieces so that neglecting them 
leads to a sizable uncertainty. Matching Eqs. (4.20) and (4.24) gives,

$$
\begin{aligned}
& \left.\bar{g}_{0}\right|_{L R}=2\left(b_{D}+b_{F}\right) F_{0}^{2} \sum_{i=1,2} \mathcal{A}_{i L R}\left(\operatorname{Im} C_{i L R}^{\text {suus }}-\operatorname{Im} C_{3+i}^{\text {sdds }}\right)+\left.\bar{g}_{0}\right|_{\text {direct }}, \\
& \left.\bar{g}_{1}\right|_{L R}=2\left(2 b_{0}+b_{D}+b_{F}\right) F_{0}^{2} \sum_{i=1,2} \mathcal{A}_{i L R} \operatorname{Im}\left(2 C_{i L R}^{\text {du ud }}+C_{i L R}^{\text {suus }}+\operatorname{Im} C_{3+i}^{s d d s}\right)+\left.\bar{g}_{1}\right|_{\text {direct }},
\end{aligned}
$$

where we indicated the contributions from $\mathcal{L}_{L R}$ by $\left.\bar{g}_{0,1}\right|_{\text {direct. }}$. In principle, we can insert values of $b_{\{0, D, F\}}$ from fits to the baryon spectrum to obtain estimates for the indirect pieces. We can improve these relations by resumming higher-order corrections $[99,100]$ and instead write

$$
\begin{aligned}
& \left.\bar{g}_{0}\right|_{L R}=-\sum_{i=1,2}\left(\operatorname{Im} C_{i L R}^{s u u s}-\operatorname{Im} C_{3+i}^{s d d}\right) \frac{r_{i}}{4} \frac{d \delta m_{N}}{d \bar{m} \varepsilon}+\left.\bar{g}_{0}\right|_{\text {direct }}, \\
& \left.\bar{g}_{1}\right|_{L R}=-\sum_{i=1,2} \operatorname{Im}\left(2 C_{i L R}^{d u u d}+C_{i L R}^{s u u s}+C_{3+i}^{s d d s}\right) \frac{r_{i}}{2} \frac{d m_{N}}{d \bar{m}}+\left.\bar{g}_{1}\right|_{\text {direct }},
\end{aligned}
$$

where $\delta m_{N}=m_{n}-m_{p}$ and $2 m_{N}=m_{n}+m_{p}$. The tadpole-induced pieces, proportional to $r_{i}$, depend on known quantities such as the nucleon sigma term $\sigma_{N}=\bar{m}\left(d m_{N} / d \bar{m}\right)=59.1 \pm$ $3.5 \mathrm{MeV}$ [101] where $\bar{m}=\left(m_{u}+m_{d}\right) / 2=3.37 \pm 0.08 \mathrm{MeV}$ [102], and the nucleon mass induced by the quark mass difference: $\left(d \delta m_{N} / d \bar{m} \varepsilon\right) \simeq \delta m_{N} /(\bar{m} \varepsilon)=(2.49 \pm 0.17 \mathrm{MeV}) /(\bar{m} \varepsilon)[103$, 104], where $\varepsilon=\left(m_{d}-m_{u}\right) /(2 \bar{m})=0.37 \pm 0.03$ [102]. The above allows for an estimate of $\bar{g}_{0,1}$ as the LECs $\mathcal{A}_{i L R}$ are known from lattice-QCD calculations. The additional unknown direct pieces were estimated to induce a $50 \%$ uncertainty in ref. [31].

The remaining sources of flavor-diagonal CPV in eq. (4.1), the quark CEDMs, enter through $\tilde{\chi}_{+}$and the $\tilde{b}_{0, D, F}$ terms, which represent the indirect and direct contributions, respectively. In this case both the direct and indirect contributions involve unknown LECs. We will therefore employ estimates resulting from QCD sum-rule calculations [105], leading to

$$
\left.\bar{g}_{0}\right|_{\mathrm{CEDM}}=-(5 \pm 10) \frac{2 F_{\pi}}{\mathrm{fm}}\left(\tilde{d}_{u}+\tilde{d}_{d}\right),\left.\quad \bar{g}_{1}\right|_{\mathrm{CEDM}}=-\left(20_{-10}^{+40}\right) \frac{2 F_{\pi}}{\mathrm{fm}}\left(\tilde{d}_{u}-\tilde{d}_{d}\right),
$$

which hold at a scale of $\mu=1 \mathrm{GeV}$. The contributions from the strange-quark CEDM are proportional to the small $\eta-\pi$ mixing angle [99] and we neglect them.

\section{Observables}

Before describing the expressions we employ in our analysis, we briefly discuss the different classes of experiments and the LR parameters they are most sensitive to.

- Leptonic and semileptonic charged-current decays.

These observables are known very accurately. For example, uncertainties on the lifetimes of superallowed $\beta$ emitters, which enter the determination of $V_{u d}$, appear at the $\mathcal{O}\left(10^{-4}\right)$ level [106]. The branching ratios for $K \rightarrow \mu \nu$ and $K \rightarrow \pi \ell \nu$ have 
uncertainties at the permille level. Leptonic and semileptonic decays of $B$ and $D$ mesons are known at the percent level. In addition, the theoretical input required to convert the observables into bounds on SM and LR parameters is only affected by small theoretical uncertainties.

Corrections to leptonic and semileptonic decays are induced at tree level, by the mixing between the left- and right-handed $W$ bosons, and are proportional to $C_{H u d} \sim$ $\xi V_{R} M_{W_{R}}^{-2}$. We must disentangle these contributions from those from the SM CKM matrix, $\sim V_{L i j}$, in order to constrain the LR parameters. We do so by exploiting measurements in different channels, sensitive to the axial-vector or vector component of the charged current. For example, purely leptonic decays of pseudoscalar mesons probe the axial-vector component of the charged current, while $0^{+} \rightarrow 0^{+}$superallowed nuclear transitions and semileptonic decays of pseudoscalar mesons are sensitive to the vector component. In this way it is possible to fit the SM CKM parameters $V_{L u j}$ and $V_{L c j}$, with $j \in\{d, s, b\}$, together with the corresponding LR contributions.

- Purely hadronic charged-current decays.

These include $\Delta S=1$ processes such as $K \rightarrow \pi \pi$, in particular $\varepsilon^{\prime}$, which measures direct $\mathrm{CP}$ violation in kaon decays, and $\Delta B=1$ processes such as $B \rightarrow J / \psi K_{S}$, $B \rightarrow \pi \pi$ and $B \rightarrow D K$, which, in the SM, contribute to the determination of the CKM parameters $\bar{\rho}$ and $\bar{\eta}$. In the mLRSM, these processes receive contributions from $W_{L}-W_{R}$ mixing, proportional to $C_{H u d}$, and from the exchange of $W_{R}$ between right-handed quarks, proportional to $C_{1 R R}$ and $C_{2} R R$. While the experimental measurements have uncertainties similar to the leptonic and semileptonic decays, theoretical uncertainties are usually much larger, so that these channels provide sensitive probes of LR parameters only if the SM contribution is suppressed. This is the case of $\varepsilon^{\prime}$, which in the SM receives contributions at one loop and is further suppressed by the small $V_{L t d}$ and $V_{L t s}$ elements. In the mLRSM, $\varepsilon^{\prime}$ receives a large mixing contributions at tree level and is sensitive to the combination $\operatorname{Im} C_{H u d}^{i j} V_{L}^{i k *} \sim$ $\xi M_{W_{R}}^{-2} \operatorname{Im}\left(V_{R}^{i j} V_{L}^{i k *} e^{i \alpha}\right)$, with $j, k \in\{d, s\}$ and $j \neq k$. The CP asymmetries in $\Delta B=1$ decays, on the other hand, arise at tree level in the SM, and are thus less sensitive to the contribution of the LR model.

- $\Delta S=1$ and $\Delta B=1$ flavor-changing-neutral-current (FCNC) processes.

These include several rare decays of $K$ and $B$ mesons, such as $B \rightarrow X_{s} \gamma, B \rightarrow \mu^{+} \mu^{-}$, $K_{L} \rightarrow \pi^{0} e^{+} e^{-}$and $K \rightarrow \pi \nu \bar{\nu}$. Both in the SM and in the LR model, these are generated through loop diagrams. For those channels sensitive to dipole operators, such as $B \rightarrow X_{s} \gamma$ and $K_{L} \rightarrow \pi^{0} e^{+} e^{-}$, the presence of a right-handed current causes the mLRSM contributions induced by $W_{L^{-}} W_{R}$ mixing to be enhanced by ratios of $m_{t} / m_{d, s, b}$, making these rare decays very sensitive to $C_{H u d}^{t i}$. Channels such as $B \rightarrow \mu^{+} \mu^{-}$and $K \rightarrow \pi \nu \bar{\nu}$ do not get contributions from dipole operators and thus do not obtain enhanced contributions in the mLRSM. With the experimental sensitivity approaching the SM level [107-109], in the near future these channels might be used for an extraction of the $V_{L t d}$ and $V_{L t s}$ CKM elements free of LR contamination. 
- Meson-antimeson oscillations.

A different source of stringent limits arise from $K-\bar{K}$ and $B-\bar{B}$ oscillations. Important examples include the meson mass differences, $\Delta m_{K, B_{d}, B_{s}}$, and $\varepsilon_{K}$ which measures $\mathrm{CP}$ violation in kaon mixing. The experimental input is very accurate, for instance uncertainties on $\Delta m_{K}$ and $\varepsilon_{K}$ are about $0.2 \%$ and $0.8 \%$, respectively. For observables dominated by short-distance contributions, such as $\varepsilon_{K}$ and the $B$-meson mass differences, the theoretical error is also under control. $\Delta m_{K}$ and the $D$ meson oscillations parameters, on the other hand, receive sizable (dominant in the case of $D$ mesons) long-distance contributions, which are hard to calculate in lattice QCD. The mLRSM gives large contributions to these observables, both at tree- and loop-level, which generally lead to strong bounds on $M_{H}$ and $M_{W_{R}}$, with less sensitivity to $\xi$. As the same observables are usually used to determine the CKM elements involving the top quark, $V_{L t i}$, we again need to fit CKM and LR parameters simultaneously.

- Electric dipole moments.

Finally, the EDMs of the neutron and diamagnetic atoms probe flavor-diagonal CP violation. While CKM contributions to EDMs are negligible [110-113], in the mLRSM EDMs receive large tree-level contributions from the mixing between leftand right-handed $W$ bosons and are sensitive to the combination $\operatorname{Im} C_{H u d}^{i j} V_{L}^{i j *} \sim$ $\xi M_{W_{R}}^{-2} \operatorname{Im}\left(V_{R}^{i j} V_{L}^{i j *} e^{i \alpha}\right)$.

We describe the most salient features of these observables and relegate details to appendix D.

\subsection{Leptonic and semileptonic decays}

Our analysis of leptonic and semileptonic decays follows closely ref. [48], with updated input on the lattice QCD calculations of mesonic decay constants and form factors, taken from ref. [49], and on the radiative corrections to nuclear decays [114, 115]. For each $u_{i} \rightarrow d_{j}$ transition, with $i \in\{u, c\}$ and $j \in\{d, s, b\}$, it is possible to find at least two independent channels, sensitive to the vector or axial-vector component of the charged-current. In the presence of $W_{L}-W_{R}$ mixing, these receive corrections of opposite sign. Schematically

$$
F_{V}\left|V_{L i j}+\frac{v^{2}}{2} C_{H u d}^{i j}\right|=O_{V, i j}^{\exp }, \quad F_{A}\left|V_{L i j}-\frac{v^{2}}{2} C_{H u d}^{i j}\right|=O_{A, i j}^{\exp },
$$

where $O_{\{V, A\}, i j}^{\exp }$ denotes the experimental input, while $F_{V}$ and $F_{A}$ denote theoretical input, such as meson decay constants or (axial) vector form factors. The values for the relevant meson decay constants and form factors are collected in table 5. The extraction of $V_{L i j}$ and $v^{2} C_{H u d}^{i j}$ is thus limited by both experimental and theoretical uncertainties.

The most relevant changes with respect to the analysis in ref. [48] correspond to the $u d$ and $u s$ channels. For the $u \rightarrow d$ transitions, the strongest constraint on the vector component comes from superallowed $0^{+} \rightarrow 0^{+}$transitions, while the leptonic decay $\pi \rightarrow \mu \nu$ probes only the axial-vector part of the current. Using theory predictions for $0^{+} \rightarrow 0^{+}$ 
transitions of refs. [114-116] along with the experimental input of refs. [117-119], we have

$$
\begin{aligned}
& 0^{+} \rightarrow 0^{+}: \quad\left|V_{L u d}+\frac{v^{2}}{2} C_{H u d}^{u d}\right|=0.97370 \pm 0.00014, \\
& \pi \rightarrow \mu \nu: \quad f_{\pi}\left|V_{L u d}-\frac{v^{2}}{2} C_{H u d}^{u d}\right|=(127.13 \pm 0.02 \pm 0.13) \mathrm{MeV},
\end{aligned}
$$

where $f_{\pi}$ is the pion decay constant.

Right-handed currents also affect the $\beta$ asymmetry in neutron decay [120,121], described by the parameter $\tilde{\lambda}$. While in the SM this parameter is determined by the ratio of the nucleon axial and vector charges, $g_{A}$ and $g_{V}$, in the mLRSM one has

$$
\tilde{\lambda}=\frac{g_{A}}{g_{V}}\left(1-\frac{v^{2} C_{H u d}^{u d}}{V_{L u d}}\right) .
$$

$\tilde{\lambda}$ is measured with error of $0.1 \%, \tilde{\lambda}=1.2754 \pm 0.0013$ [119]. The extraction of $C_{H u d}^{u d}$ is limited by the uncertainty on the lattice QCD determination of $g_{A}$. Currently, the most precise calculation quotes an error of $1 \%$ [122], so that $\pi \rightarrow \mu \nu$ still provides a stronger constraint. With a further reduction of the uncertainties by a factor of two, however, the neutron $\beta$ asymmetry will become competitive.

For the $s \rightarrow u$ transitions, semileptonic kaon decays probe the vector current, while the ratio of leptonic kaon and pion decays probe the axial interaction. From refs. [49, 123] one obtains,

$$
\begin{array}{rr}
K \rightarrow \pi l \nu_{l} \quad f_{+}^{K \pi}(0)\left|V_{L u s}+\frac{v^{2}}{2} C_{H u d}^{u s}\right| & =0.2165 \pm 0.0004, \\
K \rightarrow \mu \nu: \quad \frac{f_{K}\left|V_{L u s}-\frac{v^{2}}{2} C_{H u d}^{u s}\right|}{f_{\pi}\left|V_{L u d}-\frac{v^{2}}{2} C_{H u d}^{u d}\right|} & =0.2760 \pm 0.0004 .
\end{array}
$$

Eq. (5.2) uses a re-evaluation of the universal "inner radiative corrections" in $0^{+} \rightarrow 0^{+}$ transitions [114-116], which led to a reduction in the uncertainty and a significant shift of the central value. This resulted in a $3 \sigma$ shift of the SM determination of $\left.V_{u d}\right|_{0^{+} \rightarrow 0^{+}}$ from $0.97420 \pm 0.00021$ [124] to the value in eq. (5.2), and a resulting tension with CKM unitarity. As we will discuss in section 6.3 , this tension can in principle be solved by right-handed currents, but in the mLRSM this requires a relatively light $W_{R}$, which is ruled out by other observables. For kaon decays, a new lattice QCD calculation of $f_{+}^{K \pi}(0)$, with $N_{f}=2+1+1$ [125], reduced the error by a factor of 1.6 , and somewhat increases the tension with the SM. Here we will use the $N_{f}=2+1$ values in table 5 which lead to a less pronounced deviation from the SM.

We follow a similar strategy for the remaining elements of $V_{L}$ and $C_{H u d}$, and give the relevant expressions for the leptonic and semileptonic decays of $D$ and $B$ mesons, and for decays of the $\Lambda_{b}$ baryon, in appendix D.1. $B \rightarrow D l \nu_{l}, B \rightarrow D^{*} l \nu_{l}$ as well as the inclusive decays $B \rightarrow X_{c} l \nu_{l}$ and $\Lambda_{b} \rightarrow \Lambda_{c} \mu \nu_{\mu}$ allow one to determine the CKM parameter $A$, while $B \rightarrow \pi l \nu_{l}, B \rightarrow X_{u} l \nu_{l}, B^{+} \rightarrow \tau^{+} \nu_{\tau}$, and $\Lambda_{b} \rightarrow p \mu \nu_{\mu}$ determine $\left|V_{L u b}\right|$, which is proportional to $|\bar{\rho}-i \bar{\eta}|$. 
In addition to lifetimes and branching ratios, in the case of semileptonic decays of particles with spin it is possible to measure the triple correlation $\langle\vec{J}\rangle \cdot\left(\vec{p}_{e} \times \vec{p}_{\nu}\right)$, where $\vec{J}$ is the polarization of the decaying particle, which is sensitive to time-reversal violation [126]. This correlation has been measured in the decays of neutrons and $\Sigma$ baryons [127, 128], and can be used to constrain the imaginary part of $C_{H u d}$.

\subsection{Hadronic $\Delta S=1$ and $\Delta B=1$ charged-current processes}

This class includes hadronic decays of $K$ and $B$ mesons, such as $K \rightarrow \pi \pi, B \rightarrow \pi \pi$ and $B \rightarrow J / \psi K_{S}$. In the SM, these receive tree-level contributions from the operators $C_{1 L L}$ and $C_{2} L L$, induced by the exchange of a $W_{L}$ between quarks. In addition they can receive important contributions from strong and weak penguin diagrams [129].

The most important observable in this class is $\varepsilon^{\prime}$ that measures direct $\mathrm{CP}$ violation in $K \rightarrow \pi \pi$ decays and can be written as [130]

$$
\varepsilon^{\prime}=\frac{i e^{i\left(\delta_{2}-\delta_{0}\right)}}{\sqrt{2}}\left(\frac{\operatorname{Im} A_{2}}{\operatorname{Re} A_{0}}-\frac{\operatorname{Re} A_{2}}{\operatorname{Re} A_{0}} \frac{\operatorname{Im} A_{0}}{\operatorname{Re} A_{0}}\right) .
$$

Here $A_{0,2}$ represent the amplitudes $A_{0,2}=\frac{1}{\sqrt{2}}\left\langle(\pi \pi)_{I=0,2}|i H| K^{0}\right\rangle$, with $I$ the isospin state of the pions. We use the experimental values for the real parts of these amplitudes

$$
\operatorname{Re} A_{0}=33.201 \cdot 10^{-8} \mathrm{GeV}, \quad \operatorname{Re} A_{2}=1.479 \cdot 10^{-8} \mathrm{GeV} .
$$

In the SM, the amplitudes $A_{2}$ and $A_{0}$ are real at tree level. An imaginary part is generated by one-loop diagrams with virtual top quarks, and $\varepsilon^{\prime}$ is proportional to the imaginary part of

$$
\tau=-\frac{V_{L t s}^{*} V_{L t d}}{V_{L u s}^{*} V_{L u d}}
$$

which, in the SM [50], ${ }^{9}$

$$
\tau_{\mathrm{SM}}=(1.558(65)-0.663(33) i) \cdot 10^{-3} .
$$

The loop and CKM suppression, and the additional suppression by the $I=1 / 2$ rule, $\operatorname{Re} A_{2} / \operatorname{Re} A_{0} \sim 1 / 22$, lead us to expect a rather small value, to be compared with the experimental value

$$
\operatorname{Re}\left(\varepsilon^{\prime} / \varepsilon_{K}\right)_{\exp }=16.6(2.3) \cdot 10^{-4} .
$$

In the $\mathrm{SM}, \operatorname{Im} A_{0}$ and $\operatorname{Im} A_{2}$ are dominated by the matrix elements of strong and weak penguin operators, respectively (see, for example, the discussion in ref. [131]). Recent first-principle calculations of these matrix element in lattice QCD have significantly reduced the error of the SM prediction [50], which now reads

$$
\operatorname{Re}\left(\varepsilon^{\prime} / \varepsilon_{K}\right)_{\mathrm{SM}}=\frac{\operatorname{Im} \tau}{\operatorname{Im} \tau_{\mathrm{SM}}} \times 21.7(2.6)(6.2)(5.0) \cdot 10^{-4},
$$

\footnotetext{
${ }^{9}$ Notice that the value of $\tau_{S M}$ in eq. (5.8), given in ref. [50], differs by about $10 \%$ from the one obtained with the latest CKM fits in ref. [119]. Since in our framework we need to rescale the lattice QCD estimate of $\varepsilon^{\prime} / \varepsilon_{K}$ to allow CKM parameters to vary from their SM values, we use the same $\tau_{S M}$ as given in ref. [50].
} 
where the errors are the statistical and systematic uncertainties, with the latter broken up into isospin-conserving and isospin-violating pieces. This estimate is in good agreement with a recent reappraisal of the SM value of $\varepsilon^{\prime} / \varepsilon_{K}$ based on $\chi \mathrm{PT}$ and large- $N_{c}$, which yields [132]

$$
\operatorname{Re}\left(\varepsilon^{\prime} / \varepsilon_{K}\right)_{\mathrm{SM}}=14(5) \cdot 10^{-4} .
$$

The imaginary parts of $A_{0}$ and $A_{2}$ receive new contributions from the LR and RR operators appearing in eq. (3.14). Most of these contributions can be derived from the chiral Lagrangian discussed in section 4, the only additional terms arise from the parts of the RR operators that transform as $\mathbf{2} \mathbf{7}_{R} \times \mathbf{1}_{L}$, which were omitted in the chiral discussion of section 4. These contributions were determined in ref. [92] and, together with the other BSM contributions, give

$$
\begin{aligned}
& \operatorname{Im} A_{2}=\frac{F_{0}}{2 \sqrt{6}} \mathcal{A}_{i L R} \operatorname{Im}\left(C_{i L R}^{\text {suud }}-\left(C_{i L R}^{\text {duus }}\right)^{*}\right)+\frac{1}{12 \sqrt{3}} \mathcal{A}_{(27,1)}^{\prime} \operatorname{Im}\left(C_{1 R R}^{\text {duus }}+C_{2 R R}^{\text {duus }}\right), \\
& \operatorname{Im} A_{0}=-\frac{F_{0}}{\sqrt{3}} \mathcal{A}_{i L R} \operatorname{Im}\left(C_{i L R}^{\text {suud }}-\left(C_{i L R}^{\text {duus }}\right)^{*}\right)-\frac{\sqrt{3} F_{0}}{4}\left(m_{K}^{2}-m_{\pi}^{2}\right) \mathcal{A}_{i L L}^{(8)} \operatorname{Im}\left(C_{i R R}^{\text {duus }}\right),
\end{aligned}
$$

where $\mathcal{A}_{i L L}^{(8)}=\mathcal{A}_{i R R}^{(8)}, \mathcal{A}_{i L R}$ are given in eq. (4.14) and $\mathcal{A}_{(27,1)}^{\prime}(3 \mathrm{GeV})=0.0461(14) \mathrm{GeV}^{3}$. Here we neglected the contributions to $A_{0}$ proportional to $\mathcal{A}_{(27,1)}^{\prime}$ because, as mentioned in section 4 , these terms can be shown to be small compared to the $\boldsymbol{8}_{R} \times \mathbf{1}_{L}$ contributions.

The other observables in this class include $B \rightarrow J / \psi K_{S}, B \rightarrow \pi \pi$, and other $\Delta B=1$ decays used to determine the CKM angles $\alpha, \beta$ and $\gamma$ [119]. In appendix D.2.1 we argue that the LR contribution due to tree-level $W_{R}$ exchange to time-dependent CP asymmetry in $B \rightarrow J / \psi K_{S}$ can be neglected within current uncertainties, and thus the standard extraction of $\beta=\operatorname{Arg}\left(-V_{L c d} V_{L c b}^{*} / V_{L t d} V_{L t b}^{*}\right)$ can be used in the CKM fits. While similar considerations likely apply to other non-leptonic channels such as $B \rightarrow \pi \pi$ and $B \rightarrow D K$, used to determine $\alpha$ and $\gamma$, we do not explicitly include them in our analysis as hadronic matrix elements associated to LR contributions are not under control. Finally, the corrections to the $B_{d}^{0}$ and $B_{s}^{0}$ widths also belong to this class. We compute the mLRSM corrections in appendix D.2.5.

\section{$5.3 \Delta F=2$ processes}

We move on to observables in $B-\bar{B}$ and $K-\bar{K}$ oscillations that severely constrain the mLRSM. The experimental input on the $B-\bar{B}$ mass and width differences, $\Delta m_{d}, \Delta m_{s}$, $\Delta \Gamma^{(d)}$ and $\Delta \Gamma^{(s)}$, the $K-\bar{K}$ mass difference $\Delta m_{K}$, and $\varepsilon_{K}$, which measures CP violation in $K-\bar{K}$ mixing, are reported in table 1 . We now discuss the theoretical input, and the leading uncertainties.

\subsection{1 $B-\bar{B}$ oscillations}

For the $B_{q}$ mesons, with $q=\{d, s\}$, to good approximation we can use

$$
\Delta m_{q}=2\left|M_{12}^{(q)}\right|=\frac{\left|\left\langle\bar{B}_{q}^{0}\left|\mathcal{H}_{\mathrm{eff}}(\Delta B=2)\right| B_{q}^{0}\right\rangle\right|}{m_{B_{q}}} .
$$




\begin{tabular}{|c||c|c||c|c|}
\hline$\Delta S=2$ & $\Delta M_{K}$ & $(5.293 \pm 0.009) \mathrm{ns}^{-1}$ & $\left|\varepsilon_{K}\right|$ & $(2.228 \pm 0.011) \cdot 10^{-3}$ \\
\hline$\Delta B=2$ & $\Delta m_{d}$ & $(0.5064 \pm 0.0019) \mathrm{ps}^{-1}$ & $\Delta m_{s}$ & $17.7656 \pm 0.0057 \mathrm{ps}^{-1}$ \\
& $\Delta \Gamma^{(d)}$ & $(-1.3 \pm 6.7) \cdot 10^{-3} \mathrm{ps}^{-1}$ & $\Delta \Gamma^{(s)}$ & $(0.086 \pm 0.006) \mathrm{ps}^{-1}$ \\
& $a_{\mathrm{fs}}^{d}$ & $-0.0020 \pm 0.0016$ & $a_{\mathrm{fs}}^{s}$ & $-0.0006 \pm 0.0028$ \\
\hline \multirow{2}{*}{$\Delta B=1$} & $\mathrm{BR}\left(B \rightarrow X_{d} \gamma\right)$ & $(14.1 \pm 5.7) \cdot 10^{-6}$ & $\mathrm{BR}\left(B \rightarrow X_{s} \gamma\right)$ & $(3.32 \pm 0.15) \times 10^{-4}$ \\
& $A_{C P}\left(B \rightarrow X_{d+s} \gamma\right)$ & $0.032 \pm 0.034$ & $A_{C P}(B \rightarrow s \gamma)$ & $0.015 \pm 0.02$ \\
& & & $S_{K^{*} \gamma}$ & $-0.16 \pm 0.22$ \\
\hline
\end{tabular}

Table 1. Experimental input for the processes discussed in section 5.3 and for the $\Delta B=1$ processes discussed in appendix D.2 $[119,133,134]$. The branching ratios $\mathrm{BR}\left(B \rightarrow X_{d, s} \gamma\right)$ have a cut on the photon energy, $E_{\gamma}>1.6 \mathrm{GeV}$.

Within the SM the $\Delta B=2$ Hamiltonian involves operators of the form $\left(\bar{b}_{L} \gamma_{\mu} q_{L}\right)\left(\bar{b}_{L} \gamma^{\mu} q_{L}\right)$ that are generated through box diagrams. This leads to

$$
\left.M_{12}^{(q)}\right|_{\mathrm{SM}}=\frac{G_{F}^{2} m_{W}^{2} m_{B_{q}}}{12 \pi^{2}}\left(V_{L t q}^{*} V_{L t b}\right)^{2} f_{B_{q}}^{2} \hat{B}_{B_{q}} \eta_{B} S_{0}\left(x_{t}, x_{t}\right)
$$

with $x_{i}=m_{i}^{2} / m_{W}^{2}$ and $x_{t}$ should be evaluated at $\mu=m_{t}, \eta_{B}=0.55 \pm 0.01$ [135]. The loop function $S_{0}\left(x_{i}, x_{j}\right)=\frac{1}{4}\left(f_{1}\left(x_{i}, x_{j}\right)-f_{1}\left(0, x_{j}\right)-f_{1}\left(x_{i}, 0\right)+f_{1}(0,0)\right)$, with

$$
f_{1}\left(x_{i}, x_{j}\right)=-\frac{x_{j}^{2}\left(4-8 x_{j}+x_{j}^{2}\right)}{\left(x_{i}-x_{j}\right)\left(-1+x_{j}\right)^{2}} \log \left(x_{j}\right)+\frac{x_{i}^{2}\left(4-8 x_{i}+x_{i}^{2}\right)}{\left(-1+x_{i}\right)^{2}\left(x_{i}-x_{j}\right)} \log x_{i} .
$$

Finally, the RG-invariant bag parameter, $\hat{B}_{B_{q}}$, is related to the matrix element of the left-handed operator mentioned above, for which we use the FLAG average [49] shown in table 2 .

The BSM contributions arise from the $O_{4,5}$ operators in eq. (3.14), which are generated through exchange of heavy scalar bosons and loop diagrams involving $W_{R}$. The contributions are

$$
\left.M_{12}^{(q)}\right|_{\mathrm{LR}}=\frac{m_{B_{q}} f_{B_{q}}^{2}}{2}\left[\frac{1}{3} C_{4}^{b d b d} B_{5}\left(R_{q}(\mu)+\frac{3}{2}\right)+C_{5}^{b d b d} B_{4}\left(R_{q}(\mu)+\frac{1}{6}\right)\right]^{*}
$$

where $R_{q}(\mu)=m_{B_{q}}^{2} /\left(m_{b}(\mu)+m_{q}(\mu)\right)^{2}$ and the bag factors, related to the matrix elements of $O_{4,5}$, are shown in table 2 .

We then use the above expressions with $\Delta m_{q}=2\left|M_{12}^{(q)}\right|_{\mathrm{SM}}+\left.M_{12}^{(q)}\right|_{\mathrm{LR}} \mid$ to estimate the mass differences, which we compare with the experimental values [119] shown in table 1.

\subsection{2 $\Delta m_{K}$ and $\varepsilon_{K}$}

The mixing between $\bar{K}^{0}$ and $K^{0}$ is described by the off-diagonal matrix element,

$$
2 m_{K} M_{12}^{*}=\left\langle\bar{K}^{0}\left|H_{\text {eff }}(\Delta S=2)\right| K^{0}\right\rangle .
$$

To good approximation, the real part of this amplitude determines the kaon mass difference

$$
\Delta M_{K}=M_{K_{L}}-M_{K_{S}}=2 \operatorname{Re} M_{12},
$$




\begin{tabular}{|c|ccccc|}
\hline & $f_{B_{q}} \sqrt{\hat{B}_{B_{q}}}(\mathrm{MeV})$ & $f_{B_{q}}^{2} B_{4}\left(\mathrm{GeV}^{2}\right)$ & $f_{B_{q}}^{2} B_{5}\left(\mathrm{GeV}^{2}\right)$ & $f_{B_{q}}^{2} B_{2}\left(\mathrm{GeV}^{2}\right)$ & $f_{B_{q}}^{2} B_{3}\left(\mathrm{GeV}^{2}\right)$ \\
\hline$B_{d}^{0}-\bar{B}_{d}^{0}$ & $225(9)$ & $0.0390(28)(8)$ & $0.0361(35)(7)$ & $0.0285(26)(6)$ & $0.0402(77)(8)$ \\
$B_{s}^{0}-\bar{B}_{s}^{0}$ & $274(8)$ & $0.0534(35)(7)$ & $0.0493(36)(10)$ & $0.0421(27)(8)$ & $0.0576(77)(12)$ \\
\hline & $\hat{B}_{K}$ & $B_{4}$ & $B_{5}$ & & \\
\hline$K_{0}-\bar{K}_{0}$ & $0.7625(97)$ & $0.926(19)$ & $0.720(38)$ & & \\
\hline
\end{tabular}

Table 2. Relevant bag parameters for $B_{q}-\bar{B}_{q}$ oscillations and $K_{0}-\bar{K}_{0}$ oscillations. For $B_{q}-\bar{B}_{q}$ oscillations we use the RG-invariant definition, $\hat{B}_{B_{q}}[49]$, for the SM operator, while the bag parameter for the LR model are given in the $\overline{\mathrm{MS}}$ scheme, at the renormalization scale $\mu=m_{b}$ [136]. For $K_{0}-\bar{K}_{0}$ oscillations, $\hat{B}_{K}$ is RG-invariant [49], while $B_{4}$ and $B_{5}$ are given in the $\overline{\mathrm{MS}}$ scheme, at $\mu=3 \mathrm{GeV}$. We use the $N_{f}=2+1$ averages reported in ref. [49].

while the imaginary part is connected to $\mathrm{CP}$ violation in $\bar{K}^{0}-K^{0}$ mixing, described by $\varepsilon_{K}[129]$,

$$
\varepsilon_{K}=\frac{A\left(K_{L} \rightarrow(\pi \pi)_{I}=0\right)}{A\left(K_{S} \rightarrow(\pi \pi)_{I}=0\right)} \simeq \frac{e^{i \pi / 4}}{\sqrt{2} \Delta M_{K}}\left(\operatorname{Im} M_{12}+2 \operatorname{Re} M_{12} \frac{\operatorname{Im} A_{0}}{\operatorname{Re} A_{0}}\right),
$$

where the second equality uses the approximation $\Delta \Gamma_{K} \simeq-2 \Delta M_{K}$ [130].

The SM prediction. Starting with the SM prediction, $M_{12}$ receives both short- and long-distance contributions. The former arise from local $\Delta S=2$ operators, which appear at loop level in the SM and give rise to

$$
\left.M_{12}^{\mathrm{SM}}\right|_{S D}=\frac{G_{F}^{2} m_{W}^{2}}{12 \pi^{2}} m_{K} f_{K}^{2} \hat{B}_{K}\left(\eta_{c c} \lambda_{c}^{2} S_{0}\left(x_{c}\right)+2 \eta_{c t} \lambda_{c} \lambda_{t} S_{0}\left(x_{c}, x_{t}\right)+\eta_{t t} \lambda_{t}^{2} S_{0}\left(x_{t}\right)\right)^{*},
$$

where $\lambda_{i}=V_{L i s}^{*} V_{L i d}, x_{t}$ should be evaluated at $\mu=m_{t}$ and $x_{c}$ at $\mu=m_{c}$ and $\hat{B}_{K}$ describes the non-perturbative matrix element, given in table 2. From refs. [49, 135] we have

$$
\eta_{c c}=1.87 \pm 0.76, \quad \eta_{c t}=0.496 \pm 0.047, \quad \eta_{t t}=0.5765 \pm 0.0065,
$$

while the loop function $S_{0}$ is given in section 5.3.1. The short-distance contributions dominate in the $\mathrm{CP}$-violating observable $\varepsilon_{K}$, allowing us to write

$$
\varepsilon_{K}^{\mathrm{SM}}=\frac{e^{i \pi / 4} \kappa_{\varepsilon}}{\sqrt{2} \Delta M_{K}^{\text {expt. }}} \operatorname{Im}\left(\left.M_{12}^{\mathrm{SM}}\right|_{\mathrm{SD}}\right)
$$

where $\kappa_{\varepsilon}=0.94 \pm 0.02$ [135] takes into account long-distance contributions. In the case of $\varepsilon_{K}$, it is advantageous to use the unitarity of the CKM matrix to rewrite the contributions from $c c, c t$, and $t t$ graphs in eq. (5.3.2) in terms of $u t$ and $t t$ diagrams. This leads to [51]

$$
\left|\varepsilon_{K}^{\mathrm{SM}}\right|=\frac{G_{F}^{2} f_{K}^{2} m_{K^{0}} m_{W}^{2}}{6 \sqrt{2} \pi^{2} \Delta M_{K}} \hat{B}_{K} \kappa_{\varepsilon}\left|V_{L c b}\right|^{2} \lambda^{2} \bar{\eta}\left(\left|V_{L c b}\right|^{2}(1-\bar{\rho}) \eta_{t t} \mathcal{S}\left(x_{t}\right)-\eta_{u t} \mathcal{S}\left(x_{c}, x_{t}\right)\right),
$$

where $\lambda, \bar{\eta}$ and $\bar{\rho}$ determine the CKM matrix in the Wolfenstein parametrization [137]. The loop functions are given by

$$
\begin{aligned}
\mathcal{S}\left(x_{t}\right) & =S_{0}\left(x_{t}\right)+S_{0}\left(x_{c}\right)-2 S_{0}\left(x_{c}, x_{t}\right), \\
\mathcal{S}\left(x_{c}, x_{t}\right) & =S_{0}\left(x_{c}\right)-S_{0}\left(x_{c}, x_{t}\right),
\end{aligned}
$$


and the running factors are

$$
\begin{aligned}
\eta_{t t} & =0.55(1 \pm 4.2 \%+0.1 \%)=0.55 \pm 0.02, \\
\eta_{u t} & =0.402(1 \pm 1.3 \% \pm 0.2 \% \pm 0.2 \%)=0.402 \pm 0.005
\end{aligned}
$$

leading to a small uncertainty on $\eta_{u t}$ compared to large uncertainties in the $c c$ and $c t$ running factors, at the price of a slightly larger uncertainty on $\eta_{t t}$. We use eq. (5.22) for the SM prediction.

Unfortunately, unitarity cannot be used in the same way for the SM prediction for the real part of the amplitude that gives rise to $\Delta M_{K}$. We therefore employ Eq. (5.3.2) to obtain the SM expression for the short-distance contribution to $\Delta M_{K}$. In addition, long-distance contributions are significant in this case and lead to sizable uncertainties. We will assume no significant discrepancy between the SM and experimental measurement and simply use the experimental determination to estimate the SM prediction of $\Delta M_{K}$. We thus assign a theoretical uncertainty of $\sigma_{\mathrm{SM}}^{2}=\sigma_{\mathrm{SD}, \mathrm{SM}}^{2}+\left(\left.\Delta M_{K}\right|_{\text {expt. }}-\left.\Delta M_{K}^{\mathrm{SM}}\right|_{\mathrm{SD}}\right)^{2}$, where $\sigma_{\mathrm{SD}, \mathrm{SM}}$ is the uncertainty due to $\left.\Delta M_{K}^{\mathrm{SM}}\right|_{\mathrm{SD}}$.

The BSM contributions. Short-distance LR contributions arise through the $O_{4,5}$ operators in eq. (3.14)

$$
\left.M_{12}^{\mathrm{LR}}\right|_{S D}=\frac{m_{K} f_{K}^{2}}{2}\left(\frac{m_{K}}{m_{d}+m_{s}}\right)^{2}\left(\frac{1}{3} B_{5} C_{4}^{s d s d}+B_{4} C_{5}^{s d s d}\right)^{*},
$$

where $n_{f}=2+1$ lattice calculations of the matrix elements are given in table 2. Longdistance effects are induced by two insertions of $\Delta S=1$ operators, e.g. $C_{i L L} \times C_{i L R}$ and $C_{i L L} \times C_{i R R}$. We neglect the parts of the LL, RR operators that transform as $\mathbf{2 7}_{L, R} \times \mathbf{1}_{R, L}$, and use the $\mathbf{8}_{L, R} \times \mathbf{1}_{R, L}$ pieces to estimate these effects (see the discussion around Eq. (4.14)). The long-distance pieces can then be evaluated using the chiral Lagrangian in eq. (4.10). This gives

$$
\begin{aligned}
\left.2 m_{K} M_{12}^{\mathrm{LR}}\right|_{L D}=F_{0}^{4} G_{8} \frac{m_{K^{0}}^{2}\left(4 m_{K^{0}}^{2}-3 m_{\eta}^{2}-m_{\pi^{0}}^{2}\right)}{\left(m_{K^{0}}^{2}-m_{\pi^{0}}^{2}\right)\left(m_{K^{0}}^{2}-m_{\eta}^{2}\right)}[ & -\frac{1}{2} \mathcal{A}_{i L R}\left(C_{i L R}^{\text {suud }}+\left(C_{i L R}^{\text {duus }}\right)^{*}\right) \\
& \left.+\frac{m_{K}^{2}}{3} \mathcal{A}_{i R R}^{(8)} C_{i R R}^{\text {suud }}\right]^{*},
\end{aligned}
$$

where $G_{8}=\mathcal{A}_{i L L}^{(8)} C_{i L L} / 4$ is the coefficient of the SM operators transforming as $\mathbf{8}_{L} \times \mathbf{1}_{R}$. As in the SM [138], these contributions vanish at LO in $\chi \mathrm{PT}$ after taking into account the Gell-Mann-Okubo relation. The first contributions then arise at $\mathrm{N}^{2} \mathrm{LO}$ where loops and new LECs appear. As we do not control these LECs, we estimate the contributions by using the experimental values for the meson masses in eq. (5.26) and assign a 50\% uncertainty to this result [31].

We then estimate $\Delta M_{K}$ by using $\Delta M_{K}=\left.\Delta M_{K}\right|_{\text {expt. }}+2 \mathrm{Re} M_{12}^{\mathrm{LR}}$, with $M_{12}^{\mathrm{LR}}=$ $\left.M_{12}^{\mathrm{LR}}\right|_{S D}+\left.M_{12}^{\mathrm{LR}}\right|_{L D}$. To compute the CP violation in mixing we use $\varepsilon_{K}=\varepsilon_{K}^{\mathrm{SM}}+\varepsilon_{K}^{\mathrm{LR}}$. We rewrite Eq. (5.19)

$$
\varepsilon_{K}^{\mathrm{LR}}=\frac{e^{i \pi / 4}}{\sqrt{2}}\left(\frac{\operatorname{Im} M_{12}^{\mathrm{LR}}}{\Delta M_{K}^{\text {expt. }}}+\frac{\operatorname{Im} A_{0}^{\mathrm{LR}}}{\operatorname{Re} A_{0}^{\text {expt. }}}\right),
$$

where the mLRSM contributions to $\operatorname{Im} A_{0}$ are given by eq. (5.12). 


\begin{tabular}{|c|cccc|}
\hline$e \mathrm{~cm}$ & $d_{n}$ & $d_{p, D}$ & $d_{\mathrm{Hg}}$ & $d_{\mathrm{Ra}}$ \\
\hline current & $1.8 \cdot 10^{-26}$ & - & $6.3 \cdot 10^{-30}$ & $1.2 \cdot 10^{-23}$ \\
expected & $1.0 \cdot 10^{-28}$ & $1.0 \cdot 10^{-29}$ & $1.0 \cdot 10^{-30}$ & $1.0 \cdot 10^{-27}$ \\
\hline
\end{tabular}

Table 3. The first row shows the current 90\% C.L limits on the EDMs of the neutron [62, 140, 141], ${ }^{199} \mathrm{Hg}$ [142, 143], and ${ }^{225} \mathrm{Ra}$ [144]. The second row shows the expected sensitivities of future EDM experiments, see ref. [145].

To obtain constraints we finally compare the above theoretical expressions with the experimental measurements given in table 1 . We treat the experimental uncertainties and those due to eqs. (5.20), (5.25), and (5.26) as statistical.

As mentioned in section 3.5 our analysis of the short-distance contributions to $\Delta F=2$ observables is similar to that of refs. [22,41]. Differences arise from our use of updated lattice QCD results and a somewhat different approach to the diagrams involving intermediate $c-c$ and $c-t$ quarks. Comparing numerically to the expressions of ref. [22], we find that the heavy Higgs contributions agree to within $20 \%$ after turning off the running between $m_{W}$ and $M_{W_{R}}$. Similar agreement is found for the $W_{R}$ contributions that are due to $t-t$ diagrams, while we find the terms induced by the $c-c$ and $c-t$ graphs to be larger by a factor of $\sim 1.6$ and 3.9, respectively. Note that these contributions are only potentially significant for the kaon system, while the $B_{d, s}$ systems are dominated by the $t-t$ graphs. In addition, we take into account the RGE evolution between $M_{W_{R}}$ and $m_{W}$, the effects of which are discussed in section 3.6, with approximate formulae given in appendix E.

Apart from these different treatments of LR contributions, there are slight differences in the fitting procedures. Ref. [40] constrained LR contributions by demanding that they are smaller than a certain fraction of the SM prediction, in the case of $\varepsilon_{K}$ and $\Delta M_{K}$, while using the results of a fit that assumes BSM physics to dominantly arise in $\bar{B}-B$ oscillations [139] to constrain $M_{12}^{(d, s)}$ in the $B_{d, s}$-meson sector. Instead, we fit theoretical results for observables (including up-to-date SM predictions) directly to experimental measurements, taking into account theoretical and experimental uncertainties as described above. This allows us to incorporate the LR contributions to other flavor observables in a consistent manner, without having to assume that LR effects are dominant in a certain sector.

\section{$5.4 \Delta F=0$ observables: electric dipole moments}

EDMs set stringent limits on the CP-violating interactions within the mLRSM. Here we focus on the contributions to the EDMs of hadronic and nuclear systems, the current experimental limits of which are collected in table 3. In this section, we assume a PecceiQuinn mechanism is active. In the absence of such a mechanism, all EDMs are dominated by the induced $\bar{\theta}$ term (see section 2.4 ).

\subsubsection{Nucleon EDMs}

The EDMs of the neutron and proton receive contributions from several operators. We start with the four-quark operators, discussed in section 4 , that generate sizable pion-nucleon 
couplings. These operators give rise to direct and indirect contributions to the nucleon EDMs. The former are governed by so far unknown LECs, while the latter are due to loop diagrams involving the CP-violating pion-nucleon couplings of section 4.2. The EDMs resulting from the four-quark operators can be written as follows [146]

$$
\begin{aligned}
\left.d_{n}\right|_{L R}= & \left.\bar{d}_{n}(\mu)\right|_{L R}+\frac{\left.e g_{A} \bar{g}_{1}\right|_{L R}}{\left(4 \pi F_{\pi}\right)^{2}}\left(\frac{\left.\bar{g}_{0}\right|_{L R}}{\left.\bar{g}_{1}\right|_{L R}}\left(\log \frac{m_{\pi}^{2}}{\mu^{2}}-\frac{\pi m_{\pi}}{2 m_{N}}\right)+\frac{1}{4}\left(\kappa_{1}-\kappa_{0}\right) \frac{m_{\pi}^{2}}{m_{N}^{2}} \log \frac{m_{\pi}^{2}}{\mu^{2}}\right), \\
\left.d_{p}\right|_{L R}= & \left.\bar{d}_{p}(\mu)\right|_{L R}-\frac{\left.e g_{A} \bar{g}_{1}\right|_{L R}}{\left(4 \pi F_{\pi}\right)^{2}}\left[\frac{\left.\bar{g}_{0}\right|_{L R}}{\left.\bar{g}_{1}\right|_{L R}}\left(\log \frac{m_{\pi}^{2}}{\mu^{2}}-\frac{2 \pi m_{\pi}}{m_{N}}\right)\right. \\
& \left.-\frac{1}{4}\left(\frac{2 \pi m_{\pi}}{m_{N}}+\left(\frac{5}{2}+\kappa_{1}+\kappa_{0}\right) \frac{m_{\pi}^{2}}{m_{N}^{2}} \log \frac{m_{\pi}^{2}}{\mu^{2}}\right)\right]
\end{aligned}
$$

where $\left.\bar{g}_{0,1}\right|_{L R}$ are given in Eq. (4.25) and $\left.\bar{d}_{n, p}(\mu)\right|_{L R}$ are unknown LECs due to the direct contributions of the four-quark operators. In addition, $g_{A} \simeq 1.27$, and $\kappa_{0}=-0.12$ and $\kappa_{1}=3.7$ are related to the nucleon magnetic moments. We estimate these contributions by taking $\mu=m_{N}$ with $\bar{d}_{n, p}\left(m_{N}\right)=0$ as a central value. The impact of the associated theoretical uncertainty due to the unknown LECs was discussed in ref. [31].

In the case of the quark CEDMs both the direct and indirect contributions to the nucleon EDMs involve unknown LECs. We therefore employ QCD sum-rules estimates to estimate the total induced nucleon EDMs [84, 111, 147, 148], while we use recent QCD sum-rule [149] and quark-model [150] calculations to estimate the contributions of the Weinberg operator. In addition, the nucleon EDMs receive contributions from the remaining CP-odd interactions, namely, the quark EDMs. Assuming a Peccei-Quinn mechanism, the sum of these terms then takes the form

$$
\begin{aligned}
d_{n}= & \left.d_{n}\right|_{L R}+g_{T}^{u} d_{u}+g_{T}^{d} d_{d}+g_{T}^{s} d_{s} \\
& -(0.55 \pm 0.28) \text { e } \tilde{d}_{u}-(1.1 \pm 0.55) \text { e } \tilde{d}_{d}-20(1 \pm 0.5) \mathrm{MeV} \text { e } g_{s} C_{\tilde{G}} \\
d_{p}= & \left.d_{p}\right|_{L R}+g_{T}^{d} d_{u}+g_{T}^{u} d_{d}+g_{T}^{s} d_{s} \\
& +(1.30 \pm 0.65) e \tilde{d}_{u}+(0.60 \pm 0.30) \text { e } \tilde{d}_{d}+18(1 \pm 0.5) \mathrm{MeV} \text { e } g_{s} C_{\tilde{G}}
\end{aligned}
$$

where $d_{u}=e Q_{u} m_{u} \operatorname{Im} C_{\gamma u}^{u u}$ and $d_{q}=e Q_{q} m_{q} \operatorname{Im} C_{\gamma d}^{q q}$ for $q=d, s$. The strange CEDM induces vanishing contributions if a Peccei-Quinn mechanism is active [147]. The quark-EDM contributions have been determined by lattice QCD calculations [151-155], which give at $\mu=1 \mathrm{GeV}$

$$
g_{T}^{u}=-0.213 \pm 0.012, \quad g_{T}^{d}=0.82 \pm 0.029, \quad g_{T}^{s}=-0.0028 \pm 0.0017 .
$$

All couplings in Eq. (5.29) should be evaluated at $1 \mathrm{GeV}$.

\subsubsection{Nuclear and atomic EDMs}

We finally consider expressions for the EDMs of light nuclei and diamagnetic atoms. The EDMs in the former category are theoretically attractive as they can accurately be described in terms of the nucleon EDMs and the pion-nucleon couplings $[156,157]$. We will focus on the EDM of the deuteron in the following. Although no experimental limits have been 
set on the EDMs of light nuclei so far, there are advanced proposals to measure them in electromagnetic storage rings [158], with an expected sensitivity given in table 3.

In contrast, the EDMs of diamagnetic atoms are stringently constrained experimentally, especially that of ${ }^{199} \mathrm{Hg}$, but they are subject to much larger theoretical uncertainties. The main contributions to the EDMs of these systems are expected to arise from the nuclear Schiff moment, as there are no large enhancement factors to mitigate the Schiff screening by the electron cloud [159]. The nuclear Schiff moment obtains large contributions from the pion-nucleon couplings, $\bar{g}_{0,1}$, which, however, require complicated many-body calculations. Currently, such calculations cannot be performed with good theoretical control [160-164], leading to large nuclear uncertainties, while the contributions from the nucleon EDMs are under better control. Here we will focus on the EDMs of mercury, currently the most stringently constrained system experimentally, and radium. The experimental limit on the latter is significantly weaker than the former, but future measurements aim at improvements of several orders of magnitude.

Collecting all the above information, we use

$$
\begin{aligned}
d_{D}=(0.94 \pm 0.01)\left(d_{n}+d_{p}\right)- & {\left[(0.18 \pm 0.02) \frac{\bar{g}_{1}}{2 F_{\pi}}\right] e \mathrm{fm} } \\
d_{\mathrm{Hg}}=-(2.1 \pm 0.5) \cdot 10^{-4} & {\left[(1.9 \pm 0.1) d_{n}+(0.20 \pm 0.06) d_{p}\right.} \\
- & \left.\left(0.13_{-0.07}^{+0.5} \frac{\bar{g}_{0}}{2 F_{\pi}}+0.25_{-0.63}^{+0.89} \frac{\bar{g}_{1}}{2 F_{\pi}}\right) e \mathrm{fm}\right] \\
d_{\mathrm{Ra}}=(7.7 \pm 0.8) \cdot 10^{-4} \cdot & {\left[(-2.5 \pm 7.6) \frac{\bar{g}_{0}}{2 F_{\pi}}+(63 \pm 38) \frac{\bar{g}_{1}}{2 F_{\pi}}\right] e \mathrm{fm} }
\end{aligned}
$$

where $\bar{g}_{0,1}=\left.\bar{g}_{0,1}\right|_{L R}+\left.\bar{g}_{0,1}\right|_{\text {CEDM }}$ can be read from Eqs. (4.26) and (4.27), $d_{n, p}$ are given by Eq. (5.29), and the experimental constraints are shown in table 3. Within our analysis we estimate the EDMs by using the central values for the relevant hadronic and nuclear matrix elements and refer to refs. [31, 165] for a discussion on the impact of the associated uncertainties.

\section{$6 \quad$ Results}

After computing the observables described in the previous section we construct a $\chi^{2}$

$$
\chi^{2}=\sum_{i=\{\mathrm{obs}\}}\left(\frac{O_{i}^{\mathrm{th}}-O_{i}^{\mathrm{expt}}}{\sigma_{i}}\right)^{2},
$$

where $O_{i}^{\text {th }}$ and $O_{i}^{\text {expt }}$ are the theoretical and experimental determinations of a particular observable and $\sigma_{i}$ is determined by summing the corresponding experimental and theoretical uncertainties described in the previous section in quadrature. The $\chi^{2}$ function thus depends on the parameters appearing in the LR model, $M_{W_{R}}, M_{H}, \alpha$, and $\xi$, as well as the SM CKM elements.

Some of the LR parameters are subject to theoretical constraints. As discussed in ref. [74], the masses $M_{W_{R}}$ and $M_{H}$ are both related to the vev $v_{R}$, so that $M_{H} / M_{W_{R}}$ is given 
by the ratio of parameters in the Higgs potential and the $\mathrm{SU}(2)$ gauge coupling. As the latter is fixed from experiment, a significant hierarchy $M_{H} \gg M_{W_{R}}$ would force the parameters in the Higgs potential to become non-perturbatively large. Because our description breaks down in this part of parameter space, we focus on the region $M_{H}<8 M_{W_{R}}$. Note that if one wants to keep these parameters in the perturbative regime up to the Grand Unification scale, $\mu \sim 10^{16} \mathrm{GeV}$, stringent limits on the LR scale of $v_{R} \gtrsim 10 \mathrm{TeV}$ can be set as well [166].

Similarly, for tuned values of $\kappa^{\prime} / \kappa=\xi \simeq 1$ certain parameters in the Higgs potential would have to become non-perturbatively large, see appendix A.1. To avoid this region we take $|\xi| \leq 0.8$. The CP-violating combination of parameters, $t_{2 \beta} s_{\alpha}=\tan 2 \beta \sin \alpha$, is constrained to be $\left|t_{2 \beta} s_{\alpha}\right| \lesssim 2 m_{b} / m_{t}$ in order to reproduce the quark masses [41, 60], see appendix A.1 for more details. Finally, for the CKM elements we use the Wolfenstein parametrization, which parametrizes the CKM matrix in terms of $\lambda, A, \bar{\rho}$, and $\bar{\eta}$, and we expand the expressions up to $\mathcal{O}\left(\lambda^{6}\right)$ [137]. We then simultaneously fit the four CKM parameters along with the LR parameters.

Obtaining constraints, e.g. in the $M_{W_{R}}-M_{H}$ plane, involves marginalizing over the remaining SM and LR parameters. This minimization of the $\chi^{2}$ is performed using NLopt [167], a free/open-source library for nonlinear optimization which includes various global and local optimization algorithms. In particular, an Improved Stochastic Ranking Evolution Strategy [168] is used. To obtain fits as those depicted in figure 1, we divide the $M_{W_{R}}-M_{H}$ plane into $40 \times 40$ squares within which we marginalize over all LR and CKM parameters. For each square, $M_{W_{R}}$ and $M_{H}$ are then constrained to lie within the considered square, while the remaining parameters are varied within the ranges described above.

Before discussing the resulting constraints on the mLRSM we check our expressions by performing an analysis of the CKM parameters in the decoupling limit, $M_{H, W_{R}} \rightarrow \infty$. We find

$$
\lambda \in[0.2254,0.2267], \quad A \in[0.78,0.82], \quad \bar{\rho} \in[0.07,0.16] \quad \bar{\eta} \in[0.35,0.39],
$$

at $90 \%$ C.L. These values are similar to the results of ref. [48] and are consistent with the values advocated by the PDG [119]. The ranges found here are wider than those of ref. [119], especially in the case of $\bar{\rho}$ and $\bar{\eta}$. The reason for the weaker constraints in the SM limit is that we do not include non-leptonic $B$ decays like $B \rightarrow \pi \pi$. The evaluation of these decays in the mLRSM would require additional non-perturbative matrix elements that are not currently available.

\subsection{Analysis without a Peccei-Quinn mechanism}

We begin the analysis in the parity-conserving mLRSM without a PQ mechanism where the model itself accounts for the smallness of the CP-violating QCD vacuum angle. As discussed in section 2.4, $\bar{\theta}$ now becomes a calculable function in terms of the LR parameters. Current EDM measurements then require that the spontaneous phase $t_{2 \beta} s_{\alpha} \simeq 0$ to very good approximation and in essence transfer the strong CP problem from $\bar{\theta}$ to $\alpha$. This effectively sets $\alpha=0,{ }^{10}$ that is, the EDM constraints are so strong that they effectively

\footnotetext{
${ }^{10}$ Note that $t_{2 \beta} \rightarrow 0$ does not give rise to a different solution to the constraint $t_{2 \beta} s_{\alpha} \simeq 0$. The reason is that $\alpha$ always appears in the combination $t_{\beta} e^{i \alpha}$.
} 

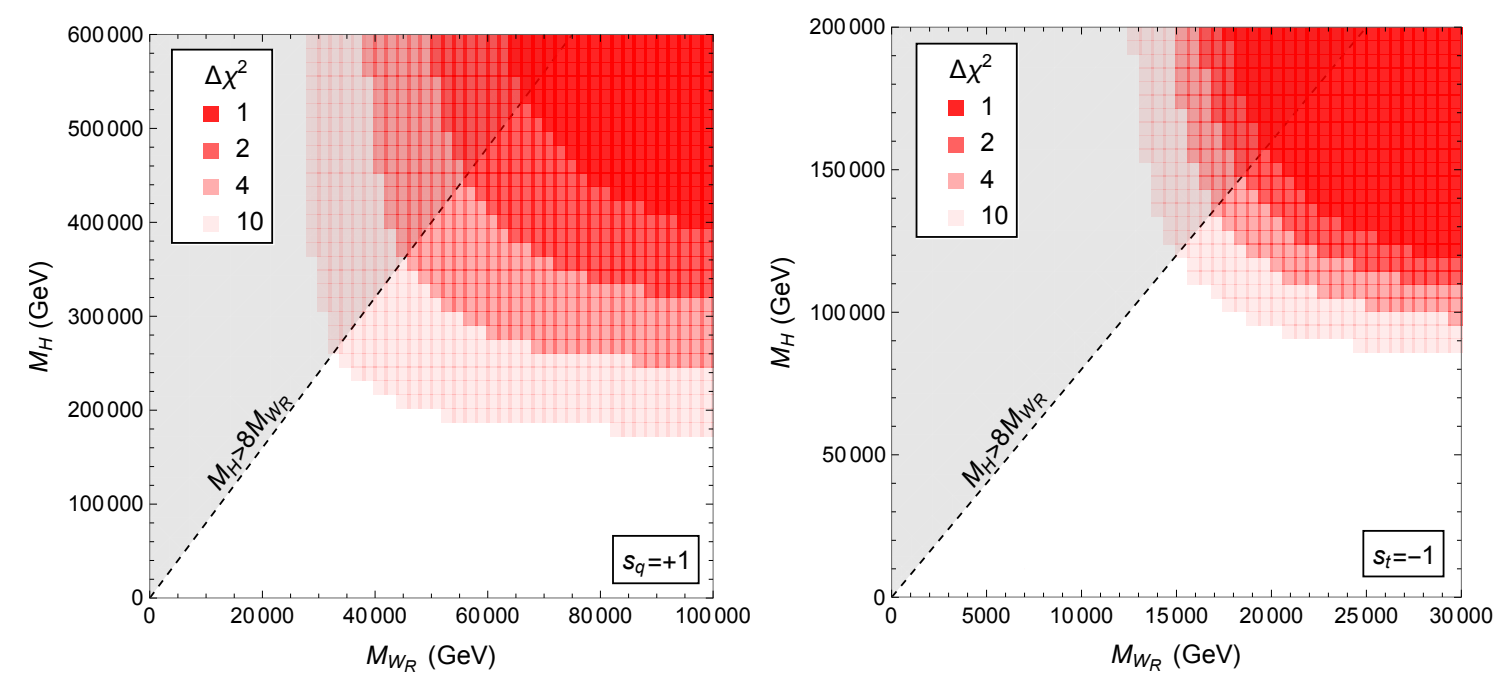

Figure 1. The left panel depicts the $\Delta \chi^{2}=\{1,2,4,10\}$ constraints in the $M_{W_{R}}-M_{H}$ plane, after marginalizing over the other LR and CKM parameters. No Peccei-Quinn mechanism is applied. The gray line shows the $M_{H}>8 M_{W_{R}}$ region where couplings in the Higgs potential become non-perturbatively large [74]. The left and right panels depict the sign configurations $s_{t}=+1$ and $s_{t}=-1$, respectively, with $s_{q \neq t}=+1$ for the remaining signs.

remove one parameter from the analysis and, after this removal, they no longer constrain the remaining parameters. We are then left with three LR parameters $\left(M_{W_{R}}, M_{H}\right.$, and $\left.\xi\right)$ and the CKM parameters that can be varied. We remind the reader that the right-handed quark-mixing matrix is expressed in terms of CKM parameters and quark masses and a set of discrete phases and reduces to $V_{R}=S_{u} V_{L} S_{d}$ in this limit, see appendix A. We begin our analysis by setting all discrete phases to $\theta_{q}=0$, and later discuss the impact of alternative sign combinations.

The main result is shown in figure 1 which depicts $\Delta \chi^{2}=\{1,2,4,10\}$ contours in the $M_{W_{R}}-M_{H}$ plane, where each point has been minimized with respect to the remaining LR and CKM parameters. The left plot illustrates a clear lower bound on $M_{W_{R}} \gtrsim 38 \mathrm{TeV}$ at $95 \%$ C.L. $\left(\Delta \chi^{2}=4\right)$ in the limit of a decoupled $M_{H} \gtrsim 400 \mathrm{TeV}$. Part of this parameter space however covers a range where the Higgs sector contains non-perturbatively large parameters. Constraining the parameter space to $M_{H}<8 M_{W_{R}}$ implies a stronger bound $M_{W_{R}} \gtrsim 45 \mathrm{TeV}$ at $95 \%$ C.L. and $M_{H}>240 \mathrm{TeV}$ at $95 \%$ C.L. for the scalar mass. The bound on $M_{W_{R}}$ is very stringent in light of the current limit on the $M_{W_{R}} \geq 4 \mathrm{TeV}$ from direct production at the LHC [169].

We still need to address the role of the sign choices, which in principle lead to 32 distinct variants of the $P$-symmetric model. It turns out that choosing $s_{i}=+1$ for all the signs leads to significantly more stringent constraints than some other assignments. For instance, setting $s_{t}=-1$ while keeping the other signs the same, leads to the right panel of figure 1 . In this case, we obtain roughly $M_{W_{R}} \gtrsim 17 \mathrm{TeV} 95 \%$ C.L. in the perturbative regime. We find that each of the 32 sign combinations essentially fall in either of the two scenarios shown in figure 1 . While the more stringently constrained scenarios give rise to 
a similar value for $\left.\chi^{2}\right|_{\min }$ as the SM, the less constrained sign combinations allow for a smaller value by about $\sim 5$. We discuss this slight improvement of the fit compared to the SM in more detail in the next subsection, in which we consider the LRM with a PQ mechanism, where a similar improvement of the fit can be achieved.

In both cases, the strong bounds are essentially driven by $\varepsilon_{K}$. This observable obtains contributions due to $\sin \alpha$ as well as mLRSM contributions proportional to the CP-odd phase in the CKM matrix that survive even when $\alpha \rightarrow 0$. A low-mass $W_{R}$ then requires cancellations to occur between these two different LR contributions to CP-violation in $K^{0}-\bar{K}^{0}$ mixing. This only becomes possible in case of a sizable spontaneous phase $\alpha[22,38,40,41]$ which is excluded in the absence of a PQ mechanism, leading to stringent limits. The $\varepsilon_{K}$ constraint is easier to satisfy for the choice $s_{t} s_{c}=-1$ and $s_{d} s_{s}=+1$ in agreement with ref. [40]. This leads to the least stringent limits and defines the class of signs depicted in the right panel of figure 1. As other observables are not as constraining, it will be difficult to further tighten the limits from low-energy constraints barring further theoretical refinements of the SM prediction of $\varepsilon_{K}$. The result $M_{W_{R}} \gtrsim 17 \mathrm{TeV}$ is still very strong compared to direct limits and is in good agreement with ref. [39] that obtained $M_{W_{R}} \gtrsim 13 \mathrm{TeV}$. The main differences with respect to our analysis is that we applied an updated SM prediction for $\varepsilon_{K}$, an improved RGE analysis, and performed a fit involving both the CKM and LR parameters.

\subsection{Analysis with a Peccei-Quinn mechanism}

We now consider the parity-conserving mLRSM in presence of a PQ mechanism. The strong $\mathrm{CP}$ problem is now resolved in the infrared and although EDMs still lead to significant constraints, they no longer effectively force $\alpha \simeq 0$. We start our analysis by setting all signs to $s_{q}=+1$. This leads to the plots in figure 2. The left panel shows $\Delta \chi^{2}=\{1,2,4,10\}$ contours in the $M_{W_{R}}-M_{H}$ plane, after marginalizing with respect to the other parameters. We thus obtain a lower bound of $M_{W_{R}} \gtrsim 5.5 \mathrm{TeV}$ at $95 \%$ C.L., in the parameter space where $M_{H}<8 M_{W_{R}}$. This limit is significantly weaker than obtained in the no-PQ scenario, where a lower bound of $M_{W_{R}} \gtrsim 38 \mathrm{TeV}$ was obtained for the same choice of discrete signs (weakened to $\sim 17 \mathrm{TeV}$ for the most favorable sign combination).

The weaker limit on $M_{W_{R}}$ compared to the scenario without a PQ mechanism is driven by the relaxed constraint on $\alpha$ and allows for a significant $t_{2 \beta} s_{\alpha} \neq 0$. As $\varepsilon_{K}$ obtains contributions from both the CKM phase and the spontaneous phase $\alpha$ cancellations between the two terms now become possible [39, 40]. This is depicted in the right panel of figure 2 where small values of $M_{W_{R}}$ clearly require a nonzero value of $t_{2 \beta} s_{\alpha}$. This rather specific value of $t_{2 \beta} s_{\alpha}$, illustrated by the funnel in the right panel leads to the mentioned cancellation which allows for much smaller values of $M_{W_{R}}$.

The lowering of the limit on $M_{W_{R}}$ only goes so far. For small $M_{W_{R}}$ other CP-violating observables like $\varepsilon^{\prime} / \varepsilon_{K}$ and EDMs become large, as these observables are induced by the CP-odd combination $t_{2 \beta} s_{\alpha}$ which is forced to be sizable by $\varepsilon_{K}$. We illustrate this in figure 3. Here we focus on the parameter space with $M_{H}=6 M_{W_{R}}$ and $M_{W_{R}}<30 \mathrm{TeV}$ as a representative example. The remaining parameters are set to the values preferred by the fit as a function of $M_{W_{R}}$. In this region, the value of $t_{2 \beta} s_{\alpha}$ then ranges between -0.009 and 

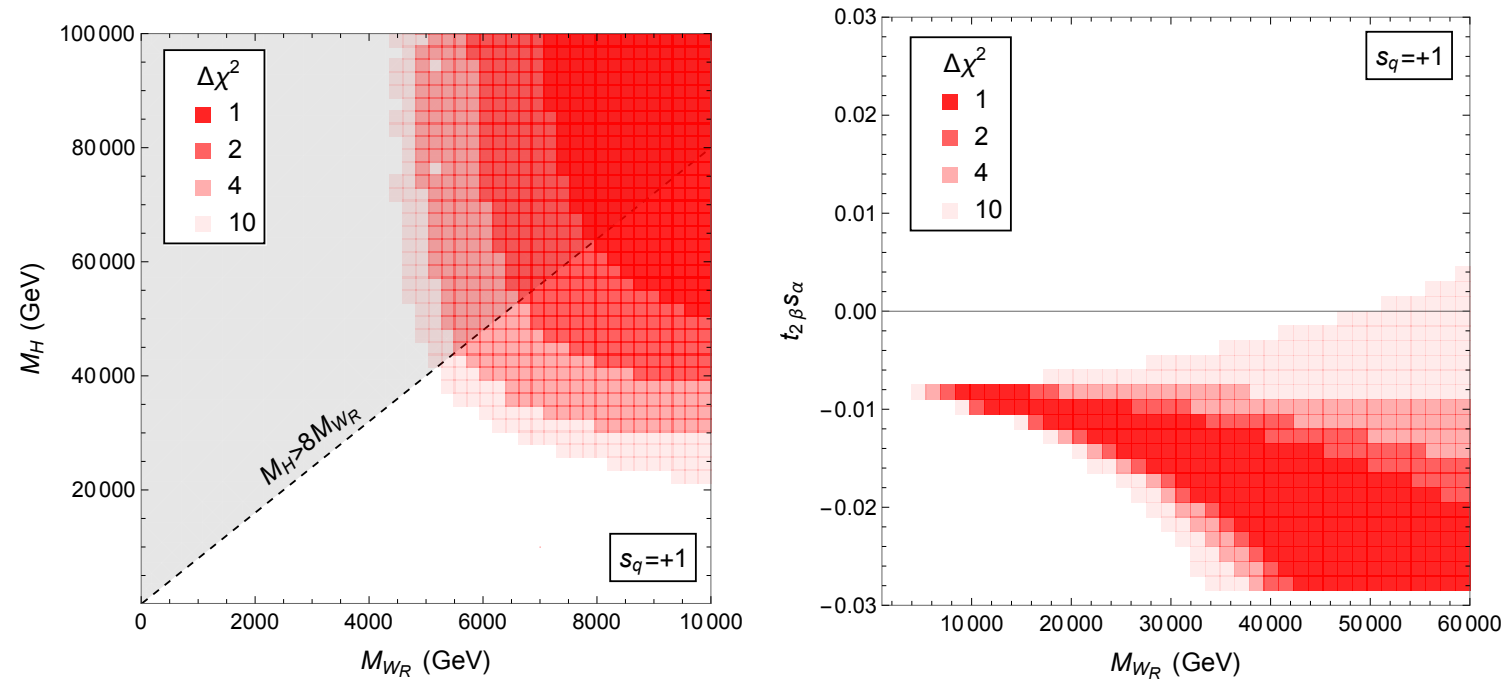

Figure 2. The left panel depicts the $\Delta \chi^{2}=\{1,2,4,10\}$ regions in the $M_{W_{R}}-M_{H}$ plane, after marginalizing over the other LR and CKM parameters. A Peccei-Quinn mechanism is applied. The gray line shows the $M_{H}>8 M_{W_{R}}$ region where couplings in the Higgs potential become nonperturbatively large [74]. The right panel shows the allowed parameter space in the $M_{W_{R}}-t_{2 \beta} s_{\alpha}$ plane for fixed $M_{H}=6 M_{W_{R}}$, while marginalizing with respect to the remaining parameters. Both panels correspond to the choice $s_{q}=+1$.
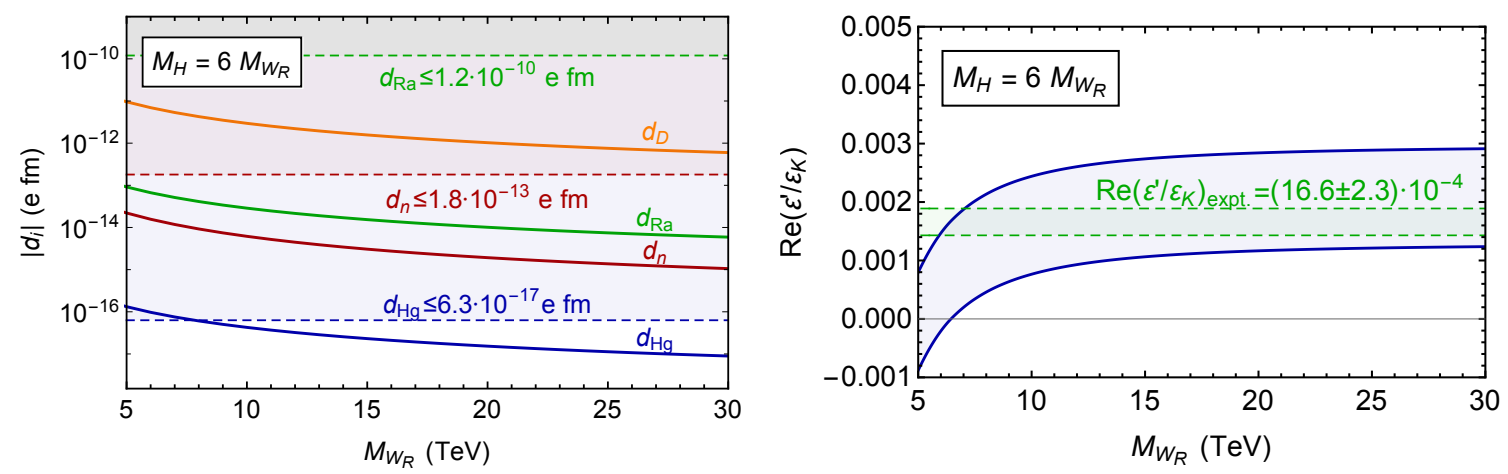

Figure 3. The left panel shows the values of various EDMs as a function of $M_{W_{R}}$ inside the 'funnel' region where $t_{2 \beta} s_{\alpha} \simeq-0.01$. The dashed lines indicate current limits. The right panel does the same for $\varepsilon^{\prime} / \varepsilon_{K}$, where the width of the blue band indicates the uncertainty of the SM prediction.

-0.014 with $t_{\beta} \simeq-0.05$ remaining constant, ${ }^{11}$ corresponding to part of the funnel region in the right panel of figure 2. We then plot values of the various EDMs as a function of $M_{W_{R}}$. The effect of the Schiff screening that affects the mercury EDM can clearly be seen from the relative sizes of $d_{n}$ and $d_{\mathrm{Hg}}$, while the relatively large values of $d_{\mathrm{Ra}}$ are due to the octupole enhancement discussed in section 5.4. The largest EDM is found to be that of the deuteron, which does not suffer from the suppression due to Schiff screening and is rather sensitive to the $\pi N$ couplings which receive large contributions in the mLRSM.

\footnotetext{
${ }^{11}$ The values of the SM CKM parameters preferred by the fit also remain roughly constant in this region, with $\lambda \simeq 0.226, A \simeq 0.79, \bar{\rho} \simeq 0.18$, and $\bar{\eta} \simeq 0.34$.
} 


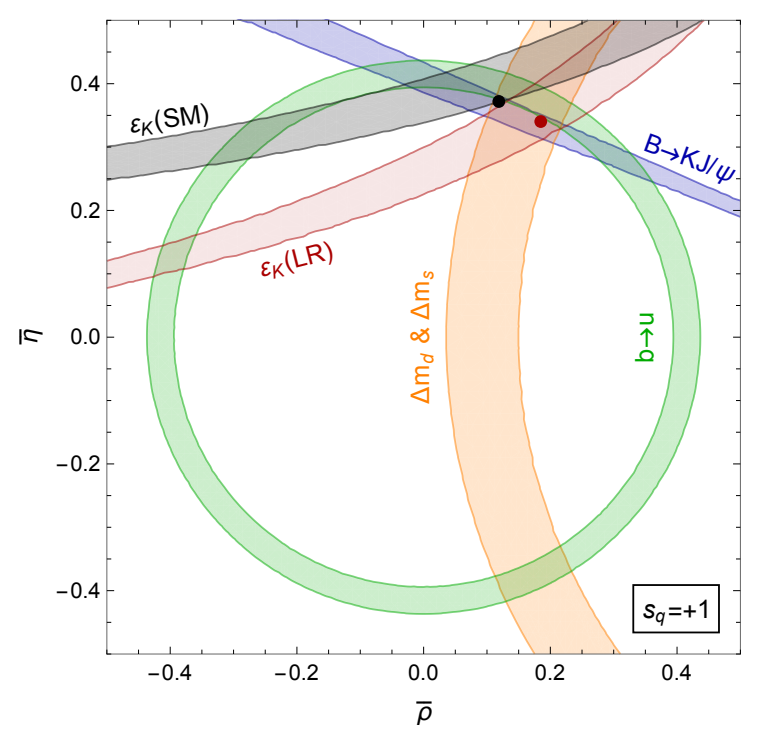

Figure 4. $68 \%$ C.L. contours from various flavor observables in the $\bar{\rho}-\bar{\eta}$ plane for two scenarios, namely the SM, $M_{H, W_{R}} \rightarrow \infty$, and the case with the best fit values for the LR parameters, $\left\{M_{H}, M_{W_{R}}, t_{2 \beta} s_{\alpha}, \alpha\right\} \simeq\{200 \mathrm{TeV}, 21 \mathrm{TeV},-0.01,3.04\}$. The difference is only noticeable in the case of $\varepsilon_{K}$ for which the SM and LRM bands are shown in black and red, respectively. Each band was obtained including $s \rightarrow u$ and $b \rightarrow c$ observables in order to marginalize with respect to $A$ and $\lambda$. The best fit points in the SM and LRM are shown as black and red points, respectively.

We observe that several EDMs are predicted to lie only one or two orders of magnitude below the present limits. That is, next-generation EDM experiments can test the funnel region corresponding to low values of $M_{W_{R}}$. For instance, a ${ }^{225}$ Ra EDM measurement at the $10^{-14}$ efm level might be possible [144] and would already go a long way in excluding small values of $M_{W_{R}}$. Similarly, a small improvement on $d_{\mathrm{Hg}}$ would have a big impact on the funnel region. Possible storage-ring experiment of $d_{D} \leq 10^{-16} \mathrm{efm}$ could have an even larger impact. We stress that a lower limit on $M_{W_{R}}$, assuming improved EDM measurements, cannot easily be deduced from the figure as it assumes values of $t_{2 \beta} s_{\alpha}$ which resulted from a fit with current experimental input. Obtaining a new lower limit on $M_{W_{R}}$ would require one to perform a new global fit once improved EDM measurements are available. The right panel of figure 3 shows that future improvements in the theoretical prediction of $\varepsilon^{\prime} / \varepsilon_{K}$, which would shrink the width of the blue band, are also excellent probes of the low $M_{W_{R}}$ regime. Apart from EDMs, there are several CP-even observables, particularly the $B$ and $K$ mass differences, which obtain significant corrections for $M_{W_{R}}$ in the $\mathrm{TeV}$ range.

Finally, we note that the fit has a slight preference for finite values of $M_{W_{R}}$ and $M_{H}$ over the SM point, $M_{H, W_{R}} \rightarrow \infty$. This is due to a mild tension in the SM fit of the CKM parameters, which can be alleviated somewhat by LR contributions to $\varepsilon_{K}$, lowering the minimum $\chi^{2}$ by roughly 5 . To illustrate the impact of the LRM we show the different experimental constraints in the $\bar{\rho}-\bar{\eta}$ plane in figure 4 , both for the SM case $\left(M_{H, W_{R}} \rightarrow\right.$ $\infty)$ and when using the best fit values for the LR parameters $\left(\left\{M_{H}, M_{W_{R}}, t_{2 \beta} s_{\alpha}, \alpha\right\} \simeq\right.$ $\{200 \mathrm{TeV}, 21 \mathrm{TeV},-0.01,3.04\}$ ). The figure shows the $68 \%$ C.L. (for two parameters, $\Delta \chi^{2}=$ 
2.3) bands for several flavor observables described in the previous sections. Each band was obtained by taking into account the $s \rightarrow u$ and $b \rightarrow c$ transitions, see section 5.1 and appendix D, and marginalizing over $A$ and $\lambda . \varepsilon_{K}$ is the only observable for which the change from the SM limit, shown in black, to the best fit point, shown in red, is noticeable. The shifted $\varepsilon_{K}$ band allows for better overlap with the preferred regions of the other observables, leading to a somewhat improved $\chi^{2}$. This change also leads to a noticeable shift in the best fit point in the $\bar{\rho}-\bar{\eta}$ plane, changing from $\{\bar{\rho}, \bar{\eta}\}=\{0.12,0.37\}$ in the SM to $\{\bar{\rho}, \bar{\eta}\}=\{0.19,0.34\}$ at the best fit point in the LRM, shown by the black and red points, respectively. Although the tension in the SM may not be very severe, the sizable shifts in the determinations of the CKM parameters due to the LRM do imply that the impact of fitting the CKM and LR parameters simultaneously can be significant.

Moving on to other possible sign choices, we find very similar allowed regions for the four cases with $s_{d} s_{s}=s_{c} s_{t}=s_{u} s_{t}=+1$, while other combinations of the signs lead to more stringent constraints and require $M_{W_{R}} \gtrsim 10 \mathrm{TeV}$ at $95 \%$ C.L. All sign combinations now allow for a lower $\left.\chi^{2}\right|_{\text {min }}$ compared to the SM, though the corresponding best fit values for the LR parameters vary. As the limits in the remaining cases are significantly tighter than those shown in figure 2 we do not further pursue the other sign choices.

\section{3 $V_{u d}, V_{u s}$, and CKM unitarity}

Before concluding we briefly discuss the discrepancy between the determinations of $V_{u d}$ and $V_{u s}$, from $0^{+} \rightarrow 0^{+}$and kaon decays, which recently sparked interest in possible BSM explanations [170-172]. The inclusion of the SM CKM parameters within our analysis enables us to consider this anomaly in a consistent manner within the mLRSM and allows one to answer whether the tension is improved by LR interactions. Before embarking on a global analysis we first consider a simpler analysis in which we focus on the observables driving the discrepancy.

The discrepancy arises from a measured value of $\left|V_{L u d}\right|^{2}+\left|V_{L u s}\right|^{2} \neq 1$, which implies a violation of unitarity (here $V_{L u b}$ is negligible with current sensitivities). Equivalently, using unitarity, one can obtain $V_{L u d}$ from the kaon decays of Eq. (5.4), which give $V_{L u d}=$ $[0.9743,0.9746]$ at $1 \sigma$. This result is in tension with the $0^{+} \rightarrow 0^{+}$determination, which, in the SM, gives $\left|V_{L u d}\right|=0.97370 \pm 0.00014$ [114-116]. Note that this discrepancy worsens if we would use the $N_{f}=2+1+1$ lattice results [49] for the form factors in Eq. (5.4) instead of the $2+1$ numbers used here.

It is interesting to see whether this discrepancy can be resolved in the mLRSM. Taking $V_{R}=S_{u} V_{L} S_{d}$, which holds to good approximation, the above mentioned observables only involve two combinations of parameters, namely, $\lambda$ and

$$
\xi_{L R} \equiv \frac{m_{W}^{2}}{M_{W_{R}}^{2}} \frac{2 \xi}{1+\xi^{2}} e^{i \alpha}
$$

As any imaginary part of $\xi_{L R}$ is stringently constrained by EDMs as well as $\varepsilon^{\prime}$, we will focus on the case where $\xi_{L R}$ is real in what follows. ${ }^{12}$ The resulting constraints from kaon decays and $0^{+} \rightarrow 0^{+}$transitions are shown in the left panel of figure 5 in blue and red, respectively.

\footnotetext{
${ }^{12}$ In addition, allowing for an imaginary part does not significantly lower the minimal $\chi^{2}$.
} 

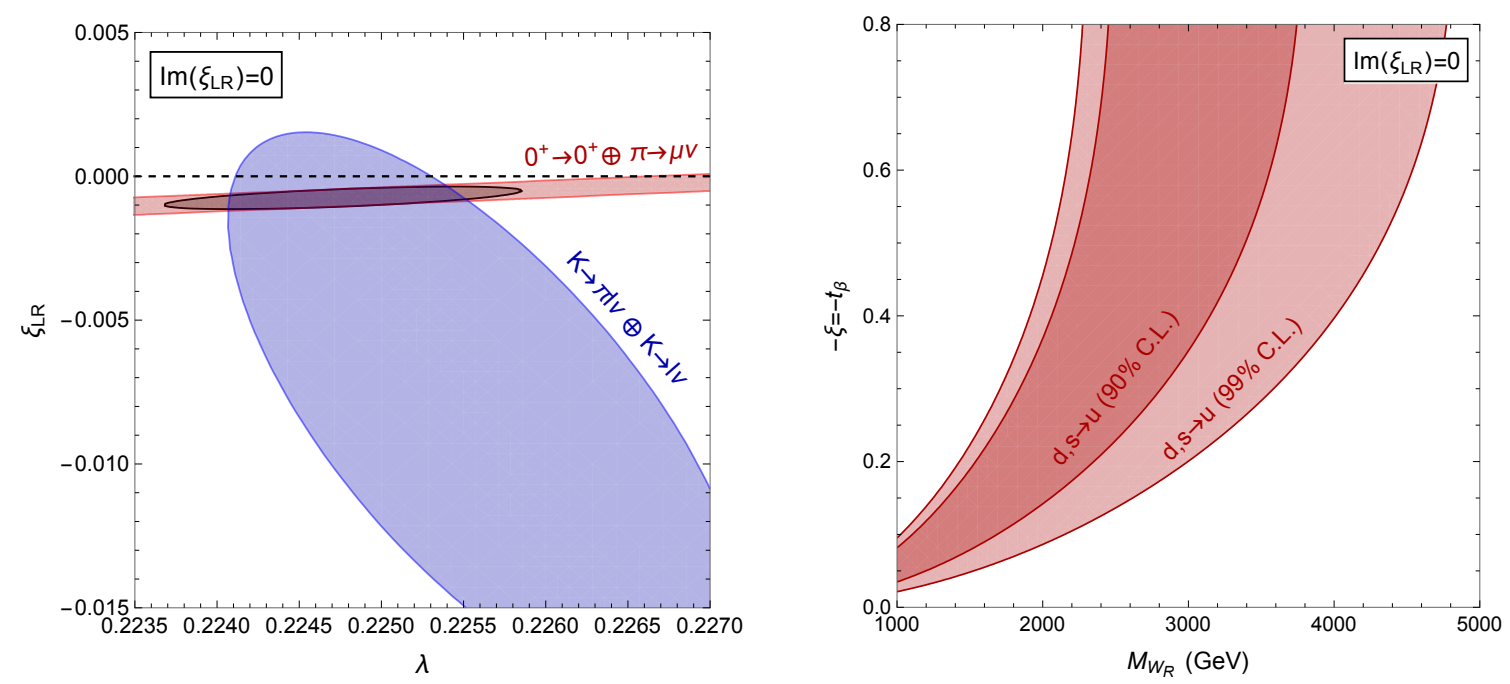

Figure 5. The left panel depicts the $\lambda-\xi_{L R}$ plane, with constraints at $90 \%$ C.L. $\left(\Delta \chi^{2}=4.6\right)$ from kaon decays in blue, those from $0^{+} \rightarrow 0^{+}$and pion decays in red, and the combination in black. The right panel shows the preferred region at $90 \%$ and $99 \%$ C.L. projected onto the $M_{W_{R}}-\xi$ plane, while allowing the SM CKM parameter $\lambda$ to vary. Both panels assume $\operatorname{Im}\left(\xi_{L R}\right)=0$.

The SM prediction is depicted by the black dashed line and it does not fit the two types of decays very well since it intersects the red and blue regions at different points. Allowing for a non-zero $\xi_{L R}$ improves the fit significantly, as the minimum $\chi^{2}$ decreases from 19 in the SM to around 3. The improvement is most significant for the sign combinations with $s_{d}=s_{s}$, as both the kaon decays and $0^{+} \rightarrow 0^{+}$prefer $\xi_{L R} V_{R}^{u d, u s} \leq 0 .{ }^{13}$ The preferred region in the $M_{W_{R}}-\xi$ plane due to the combination of $d \rightarrow u$ and $s \rightarrow u$ transitions is shown in the right panel of figure 5 , which also shows the preference for finite $M_{W_{R}}$ and $\xi$.

Thus, the mLRSM can improve the discrepancy. However, although the kaon and $0^{+} \rightarrow 0^{+}$determinations are consistent at $90 \%$ C.L. as can be seen from figure 5 , the two contours do not overlap at $1 \sigma$. The preferred size of $\operatorname{Re} \xi_{L R}$ is around $[-11,-4.5] \cdot 10^{-4}$ at $90 \%$ C.L., which implies an upper limit on $M_{W_{R}}$ of $M_{W_{R}} \lesssim 4 \mathrm{TeV}$, as can be seen from the right panel of figure 5. This value lies below the bound $M_{W_{R}} \geq 5.5 \mathrm{TeV}$ even in the presence of a PQ mechanism. Indeed, once we include other observables discussed in section 5 we find that while this region does improve the contributions from $0^{+} \rightarrow 0^{+}$and kaon decay to the total $\chi^{2}$, this improvement is offset completely by the increase due to other observables, mainly $\varepsilon_{K}$, which prefer larger values of $M_{W_{R}}$. Thus, a solution to the tension in CKM unitarity can be excluded within the $P$-symmetric mLRSM considered here. It would be interesting to see whether other variants, such as the $C$-symmetric mLRSM, can explain the discrepancy.

\footnotetext{
${ }^{13}$ The options with $s_{d}=-s_{s}$ lead to $\left.\chi^{2}\right|_{\min } \simeq 5$.
} 


\section{Conclusion}

Left-right symmetric models are promising candidates for beyond-the-SM theories that provide an origin for $P$ violation, neutrino masses, and potentially the strong $\mathrm{CP}$ problem. They also lead to a very rich phenomenology. In this work, we perform a comprehensive study of the low-energy signatures of the $P$-symmetric mLRSM. We consider the case where the model itself accounts for the smallness of $\bar{\theta}$ by requiring small spontaneous CPV phases (the no-PQ case) as well as the scenario with a Peccei-Quinn mechanism (the PQ case). The most stringent constraints on the model arise from low-energy $\beta$-decay observables, flavor observables, and EDMs. These, with the exception of EDMs, also play a large role in determining the CKM parameters so that we are forced to perform a combined fit of CKM and mLRSM parameters. We do so by including a large number of different processes for which both accurate predictions as well as measurements exist. An important role is played by low-energy probes of CP violation. We have used updated SM predictions for $\varepsilon_{K}$ and $\varepsilon^{\prime}$, using both chiral perturbation theory and lattice QCD calculations to determine mLRSM contributions. We have performed a comprehensive analysis of EDMs in the mLRSM including not just the neutron EDM, but also more complicated (and more sensitive) nuclear and atomic systems.

We note that the mLRSM does not follow the flavor structure of minimal flavor violation $(\mathrm{MFV})$ [173]. MFV requires invariance of the Lagrangian under $\mathrm{SU}(3)_{Q_{L}} \times \mathrm{SU}(3)_{u} \times \mathrm{SU}(3)_{d}$, after treating the up- and down-type Yukawa couplings as spurions transforming as $Y_{u, d} \rightarrow$ $U_{Q_{L}} Y_{u, d} U_{u, d}$. Instead, the mLRSM becomes invariant under a smaller symmetry group, $\mathrm{SU}(3)_{Q_{L}} \times \mathrm{SU}(3)_{Q_{R}}$, if one treats the Yukawa couplings as spurions that transform as $\Gamma \rightarrow U_{Q_{L}} \Gamma U_{Q_{R}}^{\dagger}$ and $\tilde{\Gamma} \rightarrow U_{Q_{L}} \tilde{\Gamma} U_{Q_{R}}^{\dagger}$. This group is less restrictive and allows for additional interactions to arise unsuppressed by small Yukawa couplings. For example, $C_{H u d}$ is induced proportional to the right-handed CKM matrix, $\sim V_{R}$, while MFV would dictate $C_{H u d} \sim Y_{u}^{\dagger} Y_{d}$. Thus, assuming MFV would lead one to expect this operator to be negligibly small, while it is actually sizable in the mLRSM and leads to important effects in a number of observable such as EDMs and $\varepsilon^{\prime}$. This implies that although the mLRSM is well suited to an EFT approach, thanks to the large hierarchy in scales $M_{W_{R}} \gg m_{W}$, it does not follow the flavor assumptions that are often employed in global SMEFT analyses. Due to the large number of operators appearing in the SMEFT, such works often take MFV as a working assumption and/or focus on high-energy collider observables [174-176]. The mLRSM is a clear example of a scenario where such an approach does not apply as it does not follow MFV, making low-energy measurements very competitive compared to direct searches for signatures of left-right models, even in a global setting.

Our main findings are summarized in figures 1 and 2 where we show constraints in the $M_{W_{R}}-M_{H}$ plane in the no-PQ and PQ case respectively. In the no-PQ case, one obtains a calculable $\bar{\theta}$ that contributes significantly to $d_{n}$ and $d_{\mathrm{Hg}}$ forcing $\alpha \ll 1$, leading to a lower bound $M_{W_{R}} \gtrsim 17 \mathrm{TeV}$ at $95 \%$ C.L. driven by $\varepsilon_{K}$. It will be hard to improve this bound with low-energy measurements unless theoretical predictions of $\varepsilon_{K}$ can significantly be improved. In the PQ case, there is no large contribution to EDMs from $\bar{\theta}$, allowing for a sizable $\alpha$. This makes it possible for contributions to $\varepsilon_{K}$ induced by $\sim \sin \alpha$ to cancel 
terms proportional to the phase in the SM CKM matrix. These cancellations weaken the constraints and we obtain $M_{W_{R}} \gtrsim 5.5 \mathrm{TeV}$ at $95 \%$ C.L., not much higher than direct limits from colliders [32-34]. This bound can be tightened significantly with next-generation EDM measurements which would essentially limit the precision with which the different contributions to $\varepsilon_{K}$ can cancel each other.

We also investigated whether the $P$-symmetric mLRSM can help resolve the CKM anomaly, finding that a relatively light $M_{W_{R}} \simeq 4 \mathrm{TeV}$ can in principle improve the tension found in the SM. Unfortunately, this region of parameter space is already excluded within a global analysis.

This work focused on low-energy observables. It would be interesting to combine the global analysis with high-energy searches. Depending on the masses of new fields this can be done either in the SMEFT framework or has to be done in the full model. In addition, we have not considered the leptonic sector of the mLRSM. The mLRSM leads to a rich phenomenology of (semi-)leptonic observables such as the electron EDM [46, 177, 178], charged-lepton flavor violation [179-181], and neutrinoless double beta decay [10, 13, 45] that can be included in a future analysis.

In conclusion, we performed a systematic and global analysis of low-energy constraints on the parity-symmetric minimal left-right symmetric model. We find no significant evidence that this model is preferred over the Standard Model and set lower bounds on the masses of right-handed gauge bosons and scalar bosons that are more stringent that direct limits.

\section{Acknowledgments}

We thank Albert Young, Leendert Hayen, and Vincenzo Cirigliano for stimulating conversations. L. A is supported by the US Department of Energy under contract DE-SC0021027. E. M. is supported by the US Department of Energy through the Office of Nuclear Physics and the LDRD program at Los Alamos National Laboratory. Los Alamos National Laboratory is operated by Triad National Security, LLC, for the National Nuclear Security Administration of U.S. Department of Energy (Contract No. 89233218CNA000001). F. O. is supported by the Dutch Organization for Scientific Research (NWO) under program 156.

\section{A Solution of $V_{R}$ in the $P$-symmetric mLRSM}

In the $P$-symmetric limit a solution for $V_{R}$ can be derived from the expressions of the mass matrices in Eq. (2.8) [60,61],

$$
M_{u}=\sqrt{1 / 2} \kappa\left(\Gamma+\xi e^{-i \alpha} \tilde{\Gamma}\right), \quad M_{d}=\sqrt{1 / 2} \kappa\left(\xi e^{i \alpha} \Gamma+\tilde{\Gamma}\right) .
$$

Both mass matrices can generally be diagonalized using two unitary matrices, $L_{q}$ and $R_{q}$, so that $M_{q}=L_{q} m_{q} R_{q}^{\dagger}$, where $m_{q}$ are real and diagonal, and the CKM matrices become $V_{L}=L_{u}^{\dagger} L_{d}$ and $V_{R}=R_{u}^{\dagger} R_{d}$. If $L_{q}$ and $R_{q}$ diagonalize the mass matrices, then the same will be true for $L_{q} S_{q}$ and $R_{q} S_{q}$, where $S_{u, d}$ are diagonal matrices of signs, meaning there will be $2^{5}$ distinct solutions for $V_{R}$. 
To determine the number of physical parameters we can note that $P$ symmetry ensures that the Yukawa matrices, $\Gamma$ and $\tilde{\Gamma}$, are hermitian, each having 9 parameters. This allows us to use a transformation of the form, $Q_{L, R} \rightarrow V Q_{L, R}$, so that $\Gamma \rightarrow V^{\dagger} \Gamma V$ becomes real and diagonal, leaving $V_{L, R}$ unchanged [41]. ${ }^{14}$ This rotation can be written as $V=V^{\prime} S$, where $V^{\prime}$ belongs to $\mathrm{SU}(3)$ and $S$ is a diagonal matrix of phases. Since $S$ is not determined by the demand that $V^{\dagger} \Gamma V=V^{\dagger} \Gamma V^{\prime}$ is diagonal, we have the freedom to use the phases in $S$ to eliminate two of the off-diagonal phases in $\tilde{\Gamma}$. Since the mass matrices determine the CKM matrices and the quark masses, this implies that $m_{q}$ and $V_{L, R}$ are a function of $\xi, \alpha$, the three parameters in $V^{\dagger} \Gamma V$, and the seven remaining parameters in $V^{\dagger} \tilde{\Gamma} V$. Conversely, this means that $V_{R}$ and the 10 parameters in $V^{\dagger} \Gamma V$ and $V^{\dagger} \tilde{\Gamma} V$ can be solved in terms of $\xi$, $\alpha$, the 6 quark masses, and the $4 \mathrm{SM}$ CKM parameters in $V_{L}$.

The above was used in refs. $[60,61]$ to obtain a solution for $V_{R}$ in terms of $\xi, \alpha, V_{L}$, and $m_{q}$. These references also obtained analytical approximations in terms of an expansion in $x \equiv \tan 2 \beta \sin \alpha$. Here we follow a similar approach as refs. [60,61] and use the hermiticity of $\Gamma$ and $\tilde{\Gamma}$ to rewrite Eq. (A.1) as,

$$
\begin{aligned}
U_{u} m_{u} U_{u}-m_{u} & =-i x\left[\xi e^{i \alpha} m_{u}-V_{L} m_{d} V_{R}^{\dagger}\right], \\
U_{d} m_{d} U_{d}-m_{d} & =i x\left[\xi e^{-i \alpha} m_{d}-V_{L}^{\dagger} m_{u} V_{R}\right], \\
V_{R} & =U_{u} V_{L} U_{d}, \quad U_{q}=L_{q}^{\dagger} R_{q} .
\end{aligned}
$$

These equations are useful as they allow one to obtain $U_{q}$ order by order after expanding both sides in terms of $x$,

$$
V_{R}=\sum_{n} x^{n} V_{R}^{(n)}, \quad U_{q}=\sum_{n} x^{n} U_{q}^{(n)},
$$

in addition, we write $\xi e^{i \alpha}=\xi \cos \alpha+i \frac{1-\xi^{2}}{2} x$. Collecting terms at each order in $x$ one can then obtain $U_{q}^{(n)}$ from the first two lines in Eq. (A.2), which now only depend on the lower order terms, $V_{R}^{(m)}$ and $U_{q}^{(m)}$, with $m<n$. The third equation in Eq. (A.2) then allows one to solve the $n$-th order in $V_{R}$ in terms of $V_{R}^{(m)}$ and $U_{q}^{(m)}$. Thus, starting with the $x^{0}$ solution, $V_{R}^{(0)}=S_{u} V_{L} S_{d}$ and $U_{q}^{(0)}=S_{q}$, any higher order can be obtained iteratively. This procedure reproduces the analytical approximations of refs. [60,61]. In our analysis we use expressions for $V_{R}$ obtained in this way and take into account terms up to and including $x^{4}$.

\section{A.1 Region of validity}

Eq. (A.1) does not allow for a solution for all values of $\xi=t_{\beta}$ and $\alpha$. A necessary condition was derived in refs. [41, 60,61], and can roughly be stated as $|x| \lesssim 2 m_{b} / m_{t}$. This condition can be obtained by considering the largest diagonal elements of the mass matrices, which we will take to be the 33 entry,

$$
\begin{aligned}
\left|\left(M_{u} M_{u}^{\dagger}\right)_{33}\right| & \geq m_{t}^{2}-\left|\left(M_{u} M_{u}^{\dagger}\right)_{31}+\left(M_{u} M_{u}^{\dagger}\right)_{32}\right| \gtrsim m_{t}^{2}-2 m_{b} m_{t}, \\
{\left[\left(M_{d}-M_{d}^{\dagger}\right) M_{u}\right]_{33} } & \lesssim 2 m_{b} m_{t},
\end{aligned}
$$

\footnotetext{
${ }^{14}$ This transformation affects the matrices needed to diagonalize the mass matrices as $L_{q} \rightarrow V^{\dagger} L_{q}$ and $R_{q} \rightarrow V^{\dagger} R_{q}$, while leaving the combinations $V_{L}=L_{u}^{\dagger} L_{d}$ and $V_{R}=R_{u}^{\dagger} R_{d}$ invariant.
} 
where the first inequality in the first line follows from eigenvalue equation for $M_{u} M_{u}^{\dagger}$. The second inequality can be derived by using that, for $i \neq j$, the matrix $\left(M_{u} M_{u}^{\dagger}\right)_{i j}$ can be expressed in terms of $M_{d} M_{d}^{\dagger}, M_{u} M_{d}^{\dagger}$, and $M_{d} M_{u}^{\dagger}$, and the fact that $\left|\left(M_{u, d}\right)_{i j}\right| \leq$ $\sum_{k} m_{u_{k}, d_{k}} \lesssim m_{t, b}$. Using the above, one can derive the following inequality in the basis where $\Gamma$ is diagonal

$$
\left|\frac{\left[\left(M_{d}-M_{d}^{\dagger}\right) M_{u}\right]_{33}}{\left[M_{u} M_{u}^{\dagger}-t_{\beta}^{2} M_{d} M_{d}^{\dagger}\right]_{33}}\right|=2\left|\frac{t_{\beta} \sin \alpha\left(1+t_{\beta} z e^{-i \alpha}\right)}{1+2 z t_{\beta}\left(1-t_{\beta}^{2}\right) \cos \alpha-t_{\beta}^{4}}\right| \lesssim 2 \frac{m_{b}}{m_{t}},
$$

where $z \equiv \Gamma_{33} / \tilde{\Gamma}_{33}$. Varying over the parameter $z$, gives a constraint that is very similar to the one discussed in ref. [41] and numerically close to $|x| \lesssim 2 m_{b} / m_{t} \simeq 0.036$. In practice, we consider the range $|x| \leq 0.03$ within which our approximate solutions of $V_{R}$ agrees with higher order solution to within $\lesssim 10 \%$.

Finally, we can see that for values of $\xi=t_{\beta} \rightarrow 1$ the Yukawa matrices have to become large in order to explain the hierarchy between the up-type and down-type masses. In particular

$$
\frac{1}{v^{2}} \operatorname{Tr}\left(M_{u} M_{u}^{\dagger}-M_{d} M_{d}^{\dagger}\right)=\frac{1}{2}\left(c_{\beta}^{2}-s_{\beta}^{2}\right) \operatorname{Tr}\left(\Gamma^{2}-\tilde{\Gamma}^{2}\right) \simeq \frac{m_{t}^{2}-m_{b}^{2}}{v^{2}},
$$

which implies that $\Gamma$ and/or $\tilde{\Gamma}$ have to become large in the $t_{\beta} \rightarrow 1$ limit. To avoid such large couplings we follow ref. [41] and restrict $\left|t_{\beta}\right|<0.8$ in our fits.

\section{B Mass eigenstates of the Higgs fields}

The spontaneous breaking of $\mathrm{SU}(2)_{L, R}$ implies that the scalar fields, $\Delta_{L, R}$ and $\phi$, should involve two neutral and two singly-charged would-be-Goldstone bosons. The remaining components are physical and make up six neutral, two singly-charged, and two doubly charged fields. The masses of these fields generally have lengthy expressions, we therefore only give approximate expressions for the $P$-symmetric case (setting some parameters in the Higgs potential to zero, $\beta_{i}=v_{L}=0$ ) and keep linear terms in $\kappa / v_{R}$ and $\xi \equiv \kappa^{\prime} / \kappa$. With these approximations the would-be-Goldstone bosons, that are absorbed by the $W_{L, R}$ and $Z_{L, R}$ bosons, can be written as

$$
\begin{array}{ll}
G_{L}^{+}=\phi_{1}^{+}-\xi e^{-i \alpha} \phi_{2}^{+}, & G_{R}^{ \pm}=\delta_{R}^{+}-\frac{\kappa}{\sqrt{2} v_{R}} \phi_{2}^{+}, \\
G_{Z}^{0}=\sqrt{2} \operatorname{Im}\left(\phi_{1}^{0 *}+\xi e^{-i \alpha} \phi_{2}^{0}\right), & G_{Z^{\prime}}^{0}=\sqrt{2} \operatorname{Im} \delta_{R}^{0} .
\end{array}
$$

The masses of the remaining (physical) states are shown in table 4, where the conventions for the parameters in the Higgs potential can be found in ref. [38].

We finally discuss the masses and mixings of the $\phi$ fields in more detail, as they play a role in section 3. Writing the bidoublet in terms of two $\mathrm{SU}(2)_{L}$ doublets, $\phi=\left(\phi_{1}, \phi_{2}\right)$, the breaking of $\mathrm{SU}(2)_{R}$, gives rise to the following mass terms,

$$
\mathcal{L} \supset-\left(\tilde{\phi}_{1}^{\dagger}, \phi_{2}^{\dagger}\right)\left(\begin{array}{cc}
v_{R}^{2} \frac{\alpha_{1}}{2}-\mu_{1}^{2} & 2 \mu_{2}^{2} e^{-i \delta_{\mu}}-\alpha_{2} v_{R}^{2} e^{i \delta_{2}} \\
2 \mu_{2}^{2} e^{i \delta_{\mu}}-\alpha_{2} v_{R}^{2} e^{-i \delta_{2}} & \frac{\alpha_{1}+\alpha_{3}}{2} v_{R}^{2}-\mu_{1}^{2}
\end{array}\right)\left(\begin{array}{c}
\tilde{\phi}_{1} \\
\phi_{2}
\end{array}\right) .
$$


Here $\alpha_{i}, \delta_{i}$, and $\mu_{i}$ are parameters of the Higgs potential, with the notation as in ref. [54]. The above terms are the $\mathcal{O}\left(v_{R}^{2}\right)$ terms for the general potential in LR models, which include the $C$ and $P$ symmetric cases (the latter has $\delta_{\mu}=0$ ). In principle the above mass matrix has 2 nonzero eigenvalues, meaning that both doublets would obtain $\mathcal{O}\left(v_{R}\right)$ masses. However, demanding that the Higgs potential resides in a minimum, $\partial V_{H} / \partial\left\{v_{R}, \kappa^{(\prime)}, \alpha\right\}=0$, give rises to,

$$
\mu_{1}^{2}-\frac{v_{R}^{2}}{2} \alpha_{1} \approx-\frac{v_{R}^{2}}{2} \frac{\xi^{2}}{1-\xi^{2}} \alpha_{3}, \quad 2 \mu_{2}^{2} e^{-i \mu_{2}}-\alpha_{2} v_{R}^{2} e^{i \delta_{2}}=\frac{1}{2} \frac{\xi \alpha_{3} v_{R}^{2}}{1-\xi^{2}} e^{-i \alpha}
$$

so that the mass terms become

$$
\mathcal{L} \supset-\left(\tilde{\phi}_{1}^{\dagger}, \phi_{2}^{\dagger}\right) \frac{\alpha_{3} v_{R}^{2}}{2\left(1-\xi^{2}\right)}\left(\begin{array}{cc}
\xi^{2} & \xi e^{-i \alpha} \\
\xi e^{i \alpha} & 1
\end{array}\right)\left(\begin{array}{l}
\tilde{\phi}_{1} \\
\phi_{2}
\end{array}\right)
$$

which can be diagonalized as in Eq. (3.3)

$$
\left(\begin{array}{c}
\tilde{\phi}_{1} \\
\phi_{2}
\end{array}\right)=\left(\begin{array}{cc}
-c_{\beta} & s_{\beta} e^{-i \alpha} \\
s_{\beta} e^{i \alpha} & c_{\beta}
\end{array}\right)\left(\begin{array}{c}
\varphi \\
\varphi_{H}
\end{array}\right),
$$

where $t_{\beta}=s_{\beta} / c_{\beta}=\xi$ and the signs are chosen such that $\langle\varphi\rangle=+\sqrt{\kappa^{2}+\kappa^{\prime 2}} / \sqrt{2}=+v / \sqrt{2}$. The mass eigenstates then have the following eigenvalues, $m_{\varphi_{S M}}^{2}=0$ and $M_{H}^{2}=\frac{\alpha_{3} v_{R}^{2}}{2} \frac{1+\xi^{2}}{1-\xi^{2}}$, which implies that the SM doublet only acquires an $\mathcal{O}\left(\kappa^{2}\right)$ mass after EWSB.

In the Higgs mass basis the Yukawa interactions of Eq. (2.3) then take the following (SU $(2)_{L}$-invariant) form,

$$
\begin{aligned}
-\mathcal{L}_{Y}= & \frac{\sqrt{2}}{v} \bar{Q}_{L}\left[\tilde{\varphi} M_{u}+\frac{1}{1-\xi^{2}} \tilde{\varphi}_{H}\left(M_{d}\left(1+\xi^{2}\right)-2 \xi e^{i \alpha} M_{u}\right)\right] U_{R} \\
& +\frac{\sqrt{2}}{v} \bar{Q}_{L}\left[\varphi M_{d}+\frac{1}{1-\xi^{2}} \varphi_{H}\left(M_{u}\left(1+\xi^{2}\right)-2 \xi e^{-i \alpha} M_{d}\right)\right] D_{R}+\text { h.c. }
\end{aligned}
$$

In the mass basis for the quarks the neutral currents become (up to $\mathcal{O}\left(\xi^{2}\right)$ terms) [38]

$$
\begin{aligned}
\mathcal{L}_{N}= & \bar{U}_{L}\left[Y_{u}\left(h^{0}-i G_{Z}^{0}\right)+\left(H_{1}^{0}-i A_{1}^{0}\right)\left(V_{L} Y_{d} V_{R}^{\dagger}-2 \xi Y_{u} e^{i \alpha}\right)\right] U_{R} \\
& +\bar{D}_{L}\left[Y_{d}\left(h^{0}+i G_{Z}^{0}\right)+\left(H_{1}^{0}+i A_{1}^{0}\right)\left(V_{L}^{\dagger} Y_{u} V_{R}-2 \xi Y_{d} e^{-i \alpha}\right)\right] D_{R}+\text { h.c. }
\end{aligned}
$$

whereas the charged scalars give rise to the following interactions,

$$
\begin{aligned}
\mathcal{L}_{C}=\sqrt{2} \bar{U}[ & \left(Y_{u} V_{R}-2 \xi e^{-i \alpha} V_{L} Y_{d}\right) P_{R} H_{2}^{+}-\left(V_{R} Y_{d}-2 \xi e^{-i \alpha} Y_{u} V_{L}\right) P_{L} H_{2}^{+} \\
& \left.+\left(Y_{u} V_{L} P_{L}-V_{L} Y_{d} P_{R}\right) G_{L}^{+}\right] D+\text { h.c. }
\end{aligned}
$$

where $Y_{u, d}$ are diagonal matrices of Yukawas, $\left(Y_{q}\right)_{i i}=m_{q_{i}} / v$. 


\begin{tabular}{|l|l|}
\hline Mass eigenstate & Mass squared \\
\hline \hline Neutral scalars & \\
\hline$h^{0}=\sqrt{2} \operatorname{Re}\left(\phi_{1}^{0 *}+\xi e^{-i \alpha} \phi_{2}^{0}\right)$ & $\frac{1}{2} \alpha_{3} v_{R}^{2} \xi^{2}+\left(2 \lambda_{1}-\frac{1}{2} \alpha_{1}^{2} / \rho_{1}\right) \kappa^{2}$ \\
$H_{1}^{0}=\sqrt{2} \operatorname{Re}\left(\phi_{2}^{0}-\xi e^{i \alpha} \phi_{1}^{0 *}\right)$ & $\frac{1}{2} \alpha_{3} v_{R}^{2}$ \\
$A_{1}^{0}=\sqrt{2} \operatorname{Im}\left(\phi_{2}^{0}-\xi e^{i \alpha} \phi_{1}^{0 *}\right)$ & $\frac{1}{2} \alpha_{3} v_{R}^{2}$ \\
$\sqrt{2} \operatorname{Re} \delta_{R}^{0}$ & $2 \rho_{1} v_{R}^{2}$ \\
$\sqrt{2} \operatorname{Re} \delta_{L}^{0}$ & $\frac{1}{2}\left(\rho_{3}-2 \rho_{1}\right) v_{R}^{2}$ \\
$\sqrt{2} \operatorname{Im} \delta_{L}^{0}$ & $\frac{1}{2}\left(\rho_{3}-2 \rho_{1}\right) v_{R}^{2}$ \\
\hline Singly-charged scalars & \\
\hline$H_{2}^{+}=\phi_{2}^{+}+\xi e^{i \alpha} \phi_{1}^{+}+\frac{\kappa}{\sqrt{2} v_{R}} \delta_{R}^{+}$ & $\frac{1}{2} \alpha_{3} v_{R}^{2}$ \\
$\delta_{L}^{+}$ & $\frac{1}{2}\left(\rho_{3}-2 \rho_{1}\right) v_{R}^{2}+\frac{1}{4} \alpha_{3} \kappa^{2}$ \\
\hline Doubly-charged scalars & \\
\hline$\delta_{R}^{++}$ & $2 \rho_{2} v_{R}^{2}$ \\
$\delta_{L}^{++}$ & $\frac{1}{2}\left(\rho_{3}-2 \rho_{1}\right) v_{R}^{2}+\frac{1}{2} \alpha_{3} \kappa^{2}$ \\
\hline
\end{tabular}

Table 4. The physical Higgs mass eigenstates and their masses for the $P$-symmetric potential, restricted to the $\beta_{i}=v_{L}=0$ case. Only linear terms in $\kappa / v_{R}$ and $\xi \equiv \kappa^{\prime} / \kappa$ have been kept [38, 182, 183]. The definitions of the parameters from the Higgs potential can be found in [38].

\section{Matching to the SMEFT in the Warsaw basis}

In section 3 we matched the mLRSM onto the SMEFT in a basis that is convenient for the discussion of low-energy observables. Here, we report the conversion between our basis and the standard "Warsaw basis" of ref. [72]. We first note that ref. [72] as well as [81, 184, 185] use a different sign convention for the gauge couplings, $g_{1,2,3}$ in their notation, and the Levi-Civita tensor. Explicitly,

$$
g^{\prime}=-g_{1}, \quad g=-g_{2}, \quad g_{s}=-g_{3},\left.\quad \epsilon^{\alpha \beta \mu \nu}\right|_{\text {Here }}=\left.\epsilon^{\alpha \beta \mu \nu}\right|_{[72]} .
$$

With these identifications, our definition of the right-handed current operator $C_{H u d}$ agrees with that of ref. [72]. For the four-quark vector and scalar operators, we find

$$
\begin{aligned}
& {\left[C_{u d}^{(1)}\right]_{\text {prst }}=-\left[C_{2 R R}+\frac{1}{N_{c}} C_{1 R R}\right]_{s r p t},} \\
& {\left[C_{u d}^{(8)}\right]_{p r s t}=-2\left[C_{1 R R}\right]_{s r p t},} \\
& {\left[C_{q u}^{(1)}\right]_{p r s t}=\left[C_{1 q u}+\frac{1}{N_{c}} C_{2, q u}\right]_{p r s t},} \\
& {\left[C_{q u}^{(8)}\right]_{p r s t}=2\left[C_{2, q u}\right]_{p r s t},} \\
& {\left[C_{q d}^{(1)}\right]_{p r s t}=\left[C_{1 q d}+\frac{1}{N_{c}} C_{2, q d}\right]_{p r s t},}
\end{aligned}
$$




$$
\begin{aligned}
{\left[C_{q d}^{(8)}\right]_{p r s t} } & =2\left[C_{2, q d}\right]_{p r s t}, \\
{\left[C_{q u q d}^{(1)}\right]_{p r s t} } & =\left[C_{1 q u q d}+\frac{1}{N_{c}} C_{2, q u q d}\right]_{p r s t}, \\
{\left[C_{q u q d}^{(8)}\right]_{p r s t} } & =2\left[C_{2, q u q d}\right]_{p r s t} .
\end{aligned}
$$

Note that a Fierz relation involving Dirac matrices was used to obtain the first two identities, so that they strictly speaking only hold at tree-level. For $d \neq 4$ the left- and right-hand sides will differ by evanescent operators, which can impact the finite parts of loop-level expressions. In practice we used the $O_{i R R}$ operators when computing the matching contributions described in section 3 , which may differ from the matching one would obtain using the SMEFT basis.

The dipole operators in Eq. (3.8) agree with the definitions of ref. [72], modulo factors of the gauge couplings,

$$
\begin{aligned}
& C_{u W}=-\frac{g}{\sqrt{2}} \Gamma_{W}^{u}, \quad C_{u B}=-\frac{g^{\prime}}{\sqrt{2}} \Gamma_{B}^{u}, \quad C_{u G}=-\frac{g_{s}}{\sqrt{2}} \Gamma_{g}^{u}, \\
& C_{d W}=-\frac{g}{\sqrt{2}} \Gamma_{W}^{d}, \quad C_{d B}=-\frac{g^{\prime}}{\sqrt{2}} \Gamma_{B}^{d}, \quad C_{d G}=-\frac{g_{s}}{\sqrt{2}} \Gamma_{g}^{d} .
\end{aligned}
$$

\section{C.1 Matching to the LEFT in the basis of ref. [1]}

Similarly, below the electroweak scale, we matched onto bases that are traditionally used in the discussion of various observables, such as meson-antimeson oscillations or $B \rightarrow X_{s} \gamma$. A complete basis for the description of low-energy observables was established in ref. [1]. Here we give the conversion between the operators introduced in section 3.3 and refs. $[1,186]$. For the gauge couplings and epsilon tensor we now have,

$$
g_{s}=-\left.g\right|_{[1]},\left.\quad e\right|_{\text {Here }}=\left.e\right|_{[1]},\left.\quad \epsilon^{\alpha \beta \mu \nu}\right|_{\text {Here }}=-\left.\epsilon^{\alpha \beta \mu \nu}\right|_{[1]} .
$$

For the four-quark operators, we find

$$
\begin{aligned}
& {\left[L_{u d}^{V 1 L L}\right]_{p r s t}=-\left[C_{2 L L}+\frac{1}{N_{c}} C_{1 L L}\right]_{s r p t},} \\
& {\left[L_{u d}^{V 8 L L}\right]_{p r s t}=-2\left[C_{1 L L}\right]_{s r p t},} \\
& {\left[L_{u d}^{V 1 R R}\right]_{p r s t}=-\left[C_{2 R R}+\frac{1}{N_{c}} C_{1 R R}\right]_{s r p t},} \\
& {\left[L_{u d}^{V 8 R R}\right]_{p r s t}=-2\left[C_{1 R R}\right]_{s r p t},} \\
& {\left[L_{u d d u}^{V 1 L R}\right]_{p r s t}=-\left[C_{1 L R}^{*}+\frac{1}{N_{c}} C_{2 L R}^{*}\right]_{r p t s},} \\
& {\left[L_{u d d u}^{V 8 L R}\right]_{p r s t}=-2\left[C_{2 L R}^{*}\right]_{r p t s},} \\
& {\left[L_{d d}^{V 1 L R}\right]_{p r s t}=\left[C_{4}+\frac{1}{N_{c}} C_{5}\right]_{p r s t},} \\
& {\left[L_{d d}^{V 8 L R}\right]_{p r s t}=2\left[C_{5}\right]_{p r s t},}
\end{aligned}
$$




\begin{tabular}{|c|c||c|c|}
\hline & Decay constant & & Form Factor \\
\hline$f_{\pi}$ & $130.2 \pm 0.8 \mathrm{MeV}$ & $f_{+}^{K \pi}(0)$ & $0.9677 \pm 0.0027$ \\
$f_{K} / f_{\pi}$ & $1.1917 \pm 0.0037$ & & \\
$f_{D}$ & $209.0 \pm 2.4 \mathrm{MeV}$ & $f_{+}^{D \pi}(0)$ & $0.666 \pm 0.029$ \\
$f_{D_{s}}$ & $248.0 \pm 1.6 \mathrm{MeV}$ & $f_{+}^{D K}(0)$ & $0.747 \pm 0.019$ \\
$f_{B}$ & $192.0 \pm 4.3 \mathrm{MeV}$ & $\mathcal{F}_{D}(1)$ & $1.035 \pm 0.040$ \\
$f_{B_{s}}$ & $228.4 \pm 3.7 \mathrm{MeV}$ & $\mathcal{F}_{D^{*}}(1)$ & $0.906 \pm 0.004 \pm 0.012$ \\
\hline
\end{tabular}

Table 5. Pseudoscalar meson decay constants and form factors as determined from lattice QCD calculations. Here we use the FLAG lattice averages with $n_{f}=2+1$ [49].

$$
\begin{aligned}
& {\left[L_{u d}^{S 1 R R}\right]_{p r s t}=\left[C_{1, q u q d}+\frac{1}{N_{c}} C_{2, q u q d}\right]_{p r v t}\left[V_{L}^{*}\right]_{v s},} \\
& {\left[L_{u d}^{S 8 R R}\right]_{p r s t}=2\left[C_{2, q u q d}\right]_{p r s t}\left[V_{L}^{*}\right]_{v s},} \\
& {\left[L_{u d d u}^{S 1 R R}\right]_{p r s t}=-\left[C_{1, q u q d}+\frac{1}{N_{c}} C_{2, q u q d}\right]_{v t p r}\left[V_{L}^{*}\right]_{v s},} \\
& {\left[L_{u d d u}^{S 8 R R}\right]_{p r s t}=-2\left[C_{2, q u q d}\right]_{v t p r}\left[V_{L}^{*}\right]_{v s},}
\end{aligned}
$$

while, for the dipole operators,

$$
\begin{array}{ll}
{\left[L_{u \gamma}\right]_{p r}=-e \frac{Q_{u}}{2} m_{u_{r}} C_{\gamma u}^{p r},} & {\left[L_{u G}\right]_{p r}=-\frac{g_{s}}{2} m_{u_{r}} C_{g u}^{p r},} \\
{\left[L_{d \gamma}\right]_{p r}=-e \frac{Q_{d}}{2} m_{d_{r}} C_{\gamma d}^{p r},} & {\left[L_{d G}\right]_{p r}=-\frac{g_{s}}{2} m_{d_{r}} C_{g d}^{p r} .}
\end{array}
$$

Finally, the Weinberg operator in LEFT is given in terms of the coefficient in Eq. (3.14) by

$$
L_{\tilde{G}}=-\frac{g_{s}}{3} C_{\tilde{G}} .
$$

\section{Observables}

In this Appendix we give the expressions for the observables that are included in our $\chi^{2}$ function, but were not discussed in the main text.

\section{D.1 Leptonic and semileptonic decays}

$\boldsymbol{u} \rightarrow \boldsymbol{d}$ and $\boldsymbol{u} \rightarrow \boldsymbol{s}$ transitions. In addition to the lifetime of superallowed $\beta$ emitters, the $\pi \rightarrow \mu \nu_{\mu}, K \rightarrow \mu \nu_{\mu}$ and $K \rightarrow \pi l \nu_{l}$ branching ratios, which were discussed in section 5.1, we use the triple correlation $\langle\vec{J}\rangle \cdot\left(\vec{p}_{e} \times \vec{p}_{\nu}\right)$, where $\vec{J}$ is the neutron or $\Sigma$ baryon polarization, which is sensitive to time-reversal violation. The mLRSM contributions to this correlation in neutron decay and $\Sigma^{-} \rightarrow n e^{-} \bar{\nu}$ can be written as [126],

$$
\begin{aligned}
D_{n} & =\frac{4 g_{A}}{1+3 g_{A}^{2}} \operatorname{Im} \frac{v^{2} C_{H u d}^{u d}}{2 V_{L u d}} \simeq 0.87 \operatorname{Im} \frac{v^{2} C_{H u d}^{u d}}{2 V_{L u d}}, \\
D_{\Sigma} & =\frac{4 g_{A \Sigma n}}{1+3 g_{A \Sigma n}^{2}} \operatorname{Im} \frac{v^{2} C_{H u d}^{u s}}{2 V_{L u s}} \simeq 1.01 \operatorname{Im} \frac{v^{2} C_{H u d}^{u s}}{2 V_{L u s}},
\end{aligned}
$$


where $g_{A}=1.27$, and $g_{A \Sigma n}=0.340 \pm 0.017$ [119] are the axial coupling of the nucleon and that of the $\Sigma$ to the neutron. The SM contribution, as well as contamination from fake $T$-odd signals from final-state interactions, are negligible with current experimental accuracy (see ref. [187] for a more detailed discussion). Current measurements give [127, 128]

$$
D_{n}=(-0.96 \pm 1.89 \pm 1.01) \cdot 10^{-4}, \quad D_{\Sigma}=0.11 \pm 0.10 .
$$

$\boldsymbol{c} \rightarrow \boldsymbol{d}$ transitions. Here we use we the leptonic and semileptonic decays of the $D$ mesons, $D^{+} \rightarrow \mu^{+} \nu_{\mu}$ and $D \rightarrow \pi l \nu_{l}$, to constrain the axial and vector couplings, respectively. The experimental input is $[119,133]$

$$
\begin{array}{rr}
D \rightarrow \pi l \nu_{l}: & f_{+}^{D \pi}(0)\left|V_{L c d}+\frac{v^{2}}{2} C_{H u d}^{c d}\right|=0.1426 \pm 0.0019, \\
D^{+} \rightarrow \mu^{+} \nu_{\mu}, \tau^{+} \nu_{\tau}: & f_{D}\left|V_{L c d}-\frac{v^{2}}{2} C_{H u d}^{c d}\right|
\end{array} \mid=45.91 \pm 1.05 \mathrm{MeV} .
$$

$c \rightarrow s$ transitions. Analogously to the $c \rightarrow d$ case, the leptonic $D_{s}$ decay and semileptonic decay of the $D$ to kaons can be used to constrain $c \rightarrow s$ transitions. We use [119, 133]

$$
\begin{array}{rr}
D \rightarrow K l \nu_{l}: \quad f_{+}^{D K}(0)\left|V_{L c s}+\frac{v^{2}}{2} C_{H u d}^{c s}\right| & =0.7226 \pm 0.0034, \\
D_{s}^{+} \rightarrow \mu^{+} \nu_{\mu}, \tau^{+} \nu_{\tau}: & f_{D_{s}}\left|V_{L c s}-\frac{v^{2}}{2} C_{H u d}^{c s}\right|
\end{array}
$$

$\boldsymbol{b} \rightarrow \boldsymbol{c}$ transitions. The vector component of the charged $W c b$ current is constrained by the semileptonic decay $B \rightarrow D l \nu_{l}$. For the axial component, the purely leptonic decay of the $B_{c}$ meson has not yet been observed, while the decay $B \rightarrow D^{*} l \nu_{l}$ depends on both the vector and axial current. In the zero-recoil limit, when $w=v \cdot v^{\prime}=1$, where $v$ and $v^{\prime}$ are the $B$ and $D$ mesons four-velocities, only the axial contribution survives [188]. Using the HFLAV averages [133], we can write

$$
\begin{aligned}
B \rightarrow D l \nu_{l}: & \eta_{E W} \mathcal{F}_{D}(1)\left|V_{L c b}+\frac{v^{2}}{2} C_{H u d}^{c b}\right|=(42.00 \pm 0.45 \pm 0.89) \cdot 10^{-3}, \\
B \rightarrow D^{*} l \nu_{l}: & \eta_{E W}^{\prime} \mathcal{F}_{D^{*}}(1)\left|V_{L c b}-\frac{v^{2}}{2} C_{H u d}^{c b}\right|=(35.27 \pm 0.11 \pm 0.36) \cdot 10^{-3},
\end{aligned}
$$

where $\eta_{E W}=1.012 \pm 0.005$ and $\eta_{E W}^{\prime}=1.0066 \pm 0.0050[119,189-191]$ are electroweak corrections and $\mathcal{F}_{D}(1)$ and $\mathcal{F}_{D^{*}}(1)$ denote the form factors, evaluated at $w=1$, which are given in table 5.

Apart from these exclusive decays, $V_{L c b}$ and $C_{H u d}^{c b}$ can also be constrained through the inclusive decays $\bar{B} \rightarrow X_{c} l \bar{\nu}_{l}$. Neglecting power corrections of order $\mathcal{O}\left(\Lambda_{\mathrm{QCD}} / m_{b}\right)$, the inclusive semileptonic width into charmed final states is given by

$$
\begin{aligned}
\Gamma\left(B \rightarrow X_{c} l \nu\right)= & \frac{G_{F}^{2} m_{b}^{5}\left|V_{L c b}\right|^{2}}{192 \pi^{3}}\left[\left(1+\left|\frac{v^{2} C_{H u d}^{c b}}{2 V_{L c b}}\right|^{2}\right)\left(1-8 \rho+8 \rho^{3}-\rho^{4}-12 \rho^{2} \log \rho\right)\right. \\
& \left.-4 \frac{m_{c}}{m_{b}} \operatorname{Re}\left(\frac{v^{2} C_{H u d}^{c b}}{2 V_{L c b}}\right)\left(1+9 \rho-9 \rho^{2}-\rho^{3}+6 \rho(1+\rho) \log \rho\right)\right], \quad \text { (D.6) }
\end{aligned}
$$


where $\rho=m_{c}^{2} / m_{b}^{2}$. We then set constraints by using the PDG average [119],

$$
B \rightarrow X_{c} l \nu: \quad\left|V_{c b}^{\mathrm{eff}}\right|=(42.2 \pm 0.8) \cdot 10^{-3},
$$

where $\left|V_{c b}^{\text {eff }}\right|^{2}=\left|V_{L c b}\right|^{2} \Gamma\left(B \rightarrow X_{c} l \nu\right) / \Gamma^{\mathrm{SM}}\left(B \rightarrow X_{c} l \nu\right)$.

The limits obtained from these inclusive decays and $B \rightarrow D^{*} l \nu_{l}$ should be interpreted as an order-of-magnitude constraint only. The reason is that Eq. (D.6) does not include power corrections [192-195], while both Eqs. (D.5) and (D.6) rely on SM fits to the leptonic and hadronic moments of the decay distributions that do not include modifications due to $C_{H u d}^{c b}$. For a recent discussion in the case of $B \rightarrow D^{*} l \nu_{l}$, see ref. [196]. A complete analysis that properly takes these issues into account is beyond the scope of the current work and we will use Eq. (D.5) and (D.6) to estimate the limits from the exclusive and inclusive measurements, while referring to refs. $[197,198]$ for a more detailed discussion.

$\boldsymbol{b} \rightarrow \boldsymbol{u}$ transitions. In the case of $b \rightarrow u$ transitions, the leptonic channel $B^{+} \rightarrow \tau^{+} \nu_{\tau}$ constrains the axial current, while the vector current is probed by $B \rightarrow \pi l \nu_{l}$. In what follows we will use the HFLAV average of the BaBar and Belle results, $\operatorname{Br}\left(B^{+} \rightarrow \tau \nu\right)=$ $(1.06 \pm 0.19) \cdot 10^{-4}[133]$, and we employ the FLAG extraction for the semileptonic case [49],

$$
\begin{aligned}
B \rightarrow \pi l \nu_{l}: & \left|V_{L u b}+\frac{v^{2}}{2} C_{H u d}^{u b}\right| & =(3.74 \pm 0.14) \cdot 10^{-3}, \\
B^{+} \rightarrow \tau^{+} \nu_{\tau}: & f_{B}\left|V_{L u b}-\frac{v^{2}}{2} C_{H u d}^{u b}\right| & =(0.77 \pm 0.12) \mathrm{MeV},
\end{aligned}
$$

where the decay constant, $f_{B}$, is given in table 5 .

In addition, inclusive decays lead to the following constraint [119],

$$
B \rightarrow X_{u} l \nu: \quad \sqrt{\left|V_{L u b}\right|^{2}+\left|\frac{v^{2}}{2} C_{H u d}^{u b}\right|^{2}}=\left(4.25 \pm 0.12_{-0.14}^{+0.15} \pm 0.23\right) \cdot 10^{-3} .
$$

These inclusive decays suffer from similar problems as those in the $b \rightarrow c$ transitions; ideally, power corrections should be included [199, 200] and the leptonic spectrum should be refitted to take into account $C_{H u d}^{u b}$ contributions. However, such an analysis is beyond the scope of the current work, and we estimate constraints from inclusive decays by using Eq. (D.9).

Finally, the measurements of $\Lambda_{b}$ baryon decays, in particular the ratio $\operatorname{Br}\left(\Lambda_{b}^{0} \rightarrow\right.$ $\left.p \mu^{-} \bar{\nu}\right)_{q^{2}>15 \mathrm{GeV}} / \operatorname{Br}\left(\Lambda_{b}^{0} \rightarrow \Lambda_{c}^{+} \mu^{-} \bar{\nu}\right)_{q^{2}>7 \mathrm{GeV}}$, are sensitive to both the $b \rightarrow u$ and $b \rightarrow c$ charged currents. Here we use the form factors from the lattice QCD calculation of ref. [201] and obtain the following partially integrated decay widths,

$$
\begin{aligned}
\Gamma\left(\Lambda_{b}^{0} \rightarrow p \mu^{-} \bar{\nu}\right)_{q^{2}>15 \mathrm{GeV}=} & 4.17 \mathrm{ps}^{-1}\left|V_{L u b}+\frac{v^{2}}{2} C_{H u d}^{u b}\right|^{2}+8.17 \mathrm{ps}^{-1}\left|V_{L u b}-\frac{v^{2}}{2} C_{H u d}^{u b}\right|^{2} \\
& \pm \sigma_{\mathrm{stat}}^{(p)} \pm \sigma_{\mathrm{syst}}^{(p)}, \\
\Gamma\left(\Lambda_{b}^{0} \rightarrow \Lambda_{c}^{+} \mu^{-} \bar{\nu}\right)_{q^{2}>7 \mathrm{GeV}=} & 1.41 \mathrm{ps}^{-1}\left|V_{L c b}+\frac{v^{2}}{2} C_{H u d}^{c b}\right|^{2}+6.99 \mathrm{ps}^{-1}\left|V_{L c b}-\frac{v^{2}}{2} C_{H u d}^{c b}\right|^{2} \\
& \pm \sigma_{\mathrm{stat}}^{\left(\Lambda_{c}^{+}\right)} \pm \sigma_{\mathrm{syst}}^{\left(\Lambda_{c}^{+}\right)}
\end{aligned}
$$


where the lattice uncertainties are given by

$$
\begin{aligned}
\left(\sigma_{\mathrm{stat}}^{(p)} \mathrm{ps}\right)^{2}= & 0.10\left|V_{L u b}+\frac{v^{2}}{2} C_{H u d}^{u b}\right|^{4}+0.33\left|V_{L u b}-\frac{v^{2}}{2} C_{H u d}^{u b}\right|^{4}+0.16\left|V_{L u b}^{2}-\left(\frac{v^{2}}{2} C_{H u d}^{u b}\right)^{2}\right|^{2}, \\
\left(\sigma_{\mathrm{syst}}^{(p)} \mathrm{ps}\right)^{2}= & 0.10\left|V_{L u b}+\frac{v^{2}}{2} C_{H u d}^{u b}\right|^{4}+0.44\left|V_{L u b}-\frac{v^{2}}{2} C_{H u d}^{u b}\right|^{4}+0.050\left|V_{L u b}^{2}-\left(\frac{v^{2}}{2} C_{H u d}^{u b}\right)^{2}\right|^{2}, \\
\left(\sigma_{\mathrm{stat}}^{\left(\Lambda_{c}^{+}\right)} \mathrm{ps}\right)^{2}= & 0.0023\left|V_{L c b}+\frac{v^{2}}{2} C_{H u d}^{c b}\right|^{4}+0.017\left|V_{L c b}-\frac{v^{2}}{2} C_{H u d}^{c b}\right|^{4} \\
& +0.0052\left|V_{L c b}^{2}-\left(\frac{v^{2}}{2} C_{H u d}^{c b}\right)^{2}\right|^{2}, \\
\left(\sigma_{\mathrm{syst}}^{\left(\Lambda_{c}^{+}\right)} \mathrm{ps}\right)^{2}= & 0.0053\left|V_{L c b}+\frac{v^{2}}{2} C_{H u d}^{c b}\right|^{4}+0.11\left|V_{L c b}-\frac{v^{2}}{2} C_{H u d}^{c b}\right|^{4} \\
& +0.0027\left|V_{L c b}^{2}-\left(\frac{v^{2}}{2} C_{H u d}^{c b}\right)^{2}\right|^{2} .
\end{aligned}
$$

We then set constraints by combining this theory prediction with the experimental determination [119, 202],

$$
\frac{\operatorname{Br}\left(\Lambda_{b}^{0} \rightarrow p \mu^{-} \bar{\nu}\right)_{q^{2}>15 \mathrm{GeV}}}{\operatorname{Br}\left(\Lambda_{b}^{0} \rightarrow \Lambda_{c}^{+} \mu^{-} \bar{\nu}\right)_{q^{2}>7 \mathrm{GeV}}}=(0.92 \pm 0.04 \pm 0.07) \cdot 10^{-2} .
$$

\section{D.2 $\Delta B=1$ and $\Delta S=1$ processes}

Here we consider two types of processes, namely decays induced at tree level through charged currents, and loop-induced flavor-changing neutral currents. The decay $B \rightarrow J / \psi K_{S}$ is in the former category and is important in the determination of the SM CKM elements. In particular, it allows for a precise determination of the phase $\beta \simeq \operatorname{Arg}\left(-V_{L t d}\right)$, while it is not expected to be very sensitive to mLRSM contributions.

Instead, $\Delta B=1$ and $\Delta S=1 \mathrm{FCNC}$ processes such as $B \rightarrow X_{s, d} \gamma$ and $K_{L} \rightarrow \pi^{0} e^{+} e^{-}$ lead to stringent constraints on the elements of $C_{H u d}$ involving the top quark, as they benefit from an enhancement factor of $m_{t} / m_{b}$ compared to the SM contributions.

The theoretical expressions for $\Delta B=1 \mathrm{FCNC}$ observables are usually written in terms of the $C_{7,8}^{(\prime)}$ coefficients, see e.g. refs. [203, 204], which are related to the couplings of the dipole operators in Eq. (3.15) as follows

$$
\begin{aligned}
C_{7}\left(m_{W}\right) & =-\frac{4 \pi^{2} Q_{d}}{V_{L t b} V_{L t q}^{*}} v^{2} C_{\gamma d}^{q b}, & C_{7}^{\prime}\left(m_{W}\right) & =-\frac{4 \pi^{2} Q_{d}}{V_{L t b} V_{L t q}^{*}} \frac{m_{q}}{m_{b}}\left(v^{2} C_{\gamma d}^{b q}\right)^{*}, \\
C_{8}\left(m_{W}\right) & =\frac{4 \pi^{2}}{V_{L t b} V_{L t q}^{*}} v^{2} C_{g d}^{q b}, & C_{8}^{\prime}\left(m_{W}\right) & =\frac{4 \pi^{2}}{V_{L t b} V_{L t q}^{*}} \frac{m_{q}}{m_{b}}\left(v^{2} C_{g d}^{b q}\right)^{*} .
\end{aligned}
$$

Below we closely follow the analysis of ref. [48] and focus on the $B \rightarrow X_{s, d} \gamma$ branching ratios, the $\mathrm{CP}$ asymmetries in inclusive $B \rightarrow X_{d, s} \gamma$ decays, and in the exclusive channel $B \rightarrow K^{* 0} \gamma$. We summarize the relevant experimental results $[119,133]$ in table 1 . 


\section{D.2.1 $B \rightarrow J / \psi K_{S}$}

In the SM, the time-dependent $\mathrm{CP}$ asymmetry in $B \rightarrow J / \psi K_{S}$ is sensitive to the angle $\beta=\operatorname{Arg}\left(-\frac{V_{L c d} V_{L c b}^{*}}{V_{L t d} V_{L t b}^{*}}\right)$. The CP asymmetry is defined as

$$
\frac{\Gamma\left(\bar{B} \rightarrow J / \psi K_{S}\right)-\Gamma\left(B \rightarrow J / \psi K_{S}\right)}{\Gamma\left(\bar{B} \rightarrow J / \psi K_{S}\right)+\Gamma\left(B \rightarrow J / \psi K_{S}\right)}=S_{J / \psi K_{S}} \sin \left(\Delta m_{d} t\right)+C_{J / \psi K_{S}} \cos \left(\Delta m_{d} t\right) .
$$

Here

$$
S_{J / \psi K_{S}}=\frac{2 \operatorname{Im} \lambda_{J / \psi K_{S}}}{1+\left|\lambda_{J / \psi K_{S}}\right|^{2}}, \quad \lambda_{J / \psi K_{S}}=\left(\frac{q}{p}\right)_{B_{d}} \frac{\bar{A}_{J / \psi K}}{A_{J / \psi K}},
$$

where $(q / p)_{B_{d}}$ is related to the mixing parameters in $B_{d}^{0}-\bar{B}_{d}^{0}$ oscillations, and the ratio of amplitudes is given by

$$
\frac{\bar{A}_{J / \psi K}}{A_{J / \psi K}}=\left(\frac{p}{q}\right)_{K} \frac{\left\langle J / \psi \bar{K}_{0}\left|\mathcal{H}_{w}\right| \bar{B}_{d}^{0}\right\rangle}{\left\langle J / \psi K_{0}\left|\mathcal{H}_{w}\right| B_{d}^{0}\right\rangle} .
$$

In both the $K-\bar{K}$ and $B-\bar{B}$ systems, the ratio $|q / p|$ can be shown to be very close to 1 without the need for additional theoretical assumptions, so that we have [205]

$$
\left(\frac{q}{p}\right)_{B_{d}}=\exp \left(i \arg \left(M_{12}^{*}\right)_{B_{d}}\right), \quad\left(\frac{q}{p}\right)_{K}=\exp \left(i \arg \left(M_{12}^{*}\right)_{K}\right),
$$

up to very small corrections. In the SM, these phases can be expressed in terms of ratios of CKM elements, while the corrections to $\left(M_{12}\right)_{B_{d}, K}$ within the mLRSM are discussed in sections 5.3.1 and 5.3.2.

In addition, there are corrections to the ratio of the $r_{J / \psi K}=\frac{\left\langle J / \psi \bar{K}_{0}\left|\mathcal{H}_{w}\right| \bar{B}_{d}^{0}\right\rangle}{\left\langle J / \psi K_{0}\left|\mathcal{H}_{w}\right| B_{d}^{0}\right\rangle}$. Within the $\mathrm{SM}$, these transitions are mediated by the tree-level charged-current operators, $C_{i L L}$. In this case, the non-perturbative matrix elements drop out in the ratio leaving only CKM elements. Within the mLRSM there are additional contributions from the $C_{i R R}$ and $C_{i L R}$ operators. Expanding the ratio to first order in $1 / M_{W_{R}}^{2}$ we have,

$$
\begin{gathered}
r_{J / \psi K}=-\frac{V_{L c b} V_{L c s}^{*}}{V_{L c b}^{*} V_{L c s}}\left[1-2 i \operatorname{Im}\left(\frac{C_{1 L R}^{b c c s}+C_{1 L R}^{c s b c}+r_{L R}\left(C_{2 L R}^{b c c s}+C_{2 L R}^{c s b c}\right)+C_{1 R R}^{b c c s}+r_{L L} C_{2 R R}^{b c c s}}{C_{1 L L}^{b c c s}+r_{L L} C_{2 L L}^{b c s}}\right)\right], \\
r_{L L}=\frac{\left\langle J / \psi \bar{K}_{0}\left|\bar{s}_{L}^{\alpha} \gamma^{\mu} c_{L}^{\beta} \bar{c}_{L}^{\beta} \gamma^{\mu} b_{L}^{\alpha}\right| \bar{B}_{d}^{0}\right\rangle}{\left\langle J / \psi \bar{K}_{0}\left|\bar{s}_{L} \gamma^{\mu} c_{L} \bar{c}_{L} \gamma^{\mu} b_{L}\right| \bar{B}_{d}^{0}\right\rangle}, \quad r_{L R}=\frac{\left\langle J / \psi \bar{K}_{0}\left|\bar{s}_{L}^{\alpha} \gamma^{\mu} c_{L}^{\beta} \bar{c}_{R}^{\beta} \gamma^{\mu} b_{R}^{\alpha}\right| \bar{B}_{d}^{0}\right\rangle}{\left\langle J / \psi \bar{K}_{0}\left|\bar{s}_{L} \gamma^{\mu} c_{L} \bar{c}_{R} \gamma^{\mu} b_{R}\right| \bar{B}_{d}^{0}\right\rangle},
\end{gathered}
$$

where the matrix elements and the Wilson coefficients are to be evaluated at the same scale. As the ratios of matrix elements, $r_{L L, L R}$, are currently unknown, the non-standard contributions to $r_{J / \psi K}$ are hard to estimate. However, these terms do not come with any enhancement factors. In addition, within the $P$-symmetric scenario, the phases of $C_{i L R, R R}$ are expected to be closely aligned to those of $C_{i L L}$ due to the relation between $V_{L}$ and $V_{R}$, Eq. (2.10), and the fact that $\alpha$ is stringently constrained by CP-violating $\Delta F=0$ observables. We therefore expect these contributions to be below the experimental sensitivity for $M_{W_{R}} \gtrsim 1 \mathrm{TeV}$ and neglect them in our analysis. We thus use $r_{J / \psi K}=-\frac{V_{L c b} V_{L c s}^{*}}{V_{L c b}^{*} V_{L c s}}$ in combination with Eqs. (D.15) and (D.16), which we compare with the experimental value [133]

$$
S_{J / \psi K_{S}}=0.695 \pm 0.019
$$




\section{D.2.2 The $B \rightarrow X_{d, s} \gamma$ branching ratio}

For the $B \rightarrow X_{d, s} \gamma$ branching ratios, we employ the expressions derived in ref. [206] rescaled by the SM predictions of refs. [207-209],

$$
\begin{aligned}
\operatorname{BR}\left(B \rightarrow X_{q} \gamma\right)= & r_{q} \frac{\mathcal{N}}{100} \frac{\left|V_{L t q}^{*} V_{L t b}\right|^{2}}{\left|V_{L c b}\right|^{2}+\left|\frac{v^{2}}{2} C_{H u d}^{c b}\right|^{2}}\left[a+a_{77}\left(\left|R_{7}\right|^{2}+\left|R_{7}^{\prime}\right|^{2}\right)+a_{7}^{r} \operatorname{Re} R_{7}+a_{7}^{i} \operatorname{Im} R_{7}\right. \\
& +a_{88}\left(\left|R_{8}\right|^{2}+\left|R_{8}^{\prime}\right|^{2}\right)+a_{8}^{r} \operatorname{Re} R_{8}+a_{8}^{i} \operatorname{Im} R_{8}+a_{\epsilon \epsilon}\left|\epsilon_{q}\right|^{2}+a_{\epsilon}^{r} \operatorname{Re} \epsilon_{q} \\
& +a_{\epsilon}^{i} \operatorname{Im} \epsilon_{q}+a_{87}^{r} \operatorname{Re}\left(R_{8} R_{7}^{*}+R_{8}^{\prime} R_{7}^{\prime *}\right)+a_{87}^{i} \operatorname{Im}\left(R_{8} R_{7}^{*}+R_{8}^{\prime} R_{7}^{\prime *}\right) \\
& \left.\left.+a_{7 \epsilon}^{r} \operatorname{Re}\left(R_{7} \epsilon_{q}^{*}\right)+a_{7 \epsilon}^{i} \operatorname{Im}\left(R_{7} \epsilon_{q}^{*}\right)+a_{8 \epsilon}^{r} \operatorname{Re}\left(R_{8} \epsilon_{q}^{*}\right)+a_{8 \epsilon}^{i} \operatorname{Im}\left(R_{8} \epsilon_{q}^{*}\right)\right], \quad \text { (D.2 }\right)
\end{aligned}
$$

where $R_{7,8}=\frac{C_{7,8}\left(m_{t}\right)}{C_{7,8}^{\mathrm{SM}}\left(m_{t}\right)}, R_{7,8}^{\prime}=\frac{C_{7,8}^{\prime}\left(m_{t}\right)}{C_{7,8}^{\mathrm{SM}}\left(m_{t}\right)}, C_{7}^{\mathrm{SM}}\left(m_{t}\right)=-0.189$, and $C_{8}^{\mathrm{SM}}\left(m_{t}\right)=-0.095$ and we neglect the SM contributions to $C_{7,8}^{\prime}$ which are suppressed by $m_{q} / m_{b}$. In addition, $\mathcal{N}=2.567(1 \pm 0.064) \cdot 10^{-3}$, while $r_{q}$ are factors that rescale the above expression to the SM predictions of refs. [207-209] for which we use $r_{s}=\frac{3.36}{3.55}$ and $r_{d}=\frac{1.73}{1.47}$. Finally, $\epsilon_{q}=\frac{V_{L u q}^{*} V_{L u b}}{V_{L t q}^{*} V_{L t b}}$ and the coefficients $a_{i j}$ can be found in ref. [206]. We applied the expressions valid for a cut on the photon energy of $E_{\gamma}>1.6 \mathrm{GeV}$, which, for $B \rightarrow X_{d} \gamma$, requires extrapolating the branching ratio quoted in ref. [133], as discussed in ref. [208].

To set constraints we compare the branching ratios in Eq. (D.20) with the current experimental world averages $[119,133]$, shown in table 1 . To take into account theoretical uncertainties, we follow refs. [203, 204] and use the following theory errors $\sigma_{d}=\frac{0.22}{1.73} \mathrm{BR}(B \rightarrow$ $\left.X_{d} \gamma\right)$ and $\sigma_{s}=\frac{0.23}{3.36} \mathrm{BR}\left(B \rightarrow X_{s} \gamma\right)$, which are added in quadrature to the experimental ones.

\section{D.2.3 The $B \rightarrow X_{d, s} \gamma$ CP asymmetry}

The $B \rightarrow X_{s} \gamma \mathrm{CP}$ asymmetry provides a probe of the phase of the $t b$ element of $C_{H u d}$. We employ the expression derived in ref. [210],

$$
\begin{aligned}
\frac{A_{C P}(B \rightarrow s \gamma)}{\pi} \equiv & \frac{1}{\pi} \frac{\Gamma\left(\bar{B} \rightarrow X_{s} \gamma\right)-\Gamma\left(B \rightarrow X_{\bar{s}} \gamma\right)}{\Gamma\left(\bar{B} \rightarrow X_{s} \gamma\right)+\Gamma\left(B \rightarrow X_{\bar{s}} \gamma\right)} \\
\approx & {\left[\left(\frac{40}{81}-\frac{40}{9} \frac{\Lambda_{c}}{m_{b}}\right) \frac{\alpha_{s}}{\pi}+\frac{\Lambda_{17}^{c}}{m_{b}}\right] \operatorname{Im} \frac{C_{2}}{C_{7}}-\left(\frac{4 \alpha_{s}}{9 \pi}+4 \pi \alpha_{s} \frac{\Lambda_{78}}{3 m_{b}}\right) \operatorname{Im} \frac{C_{8}}{C_{7}} } \\
& -\left(\frac{\Lambda_{17}^{u}-\Lambda_{17}^{c}}{m_{b}}+\frac{40}{9} \frac{\Lambda_{c}}{m_{b}} \frac{\alpha_{s}}{\pi}\right) \operatorname{Im}\left(\epsilon_{s} \frac{C_{2}}{C_{7}}\right),
\end{aligned}
$$

where the Wilson coefficients should be evaluated at the factorization scale $\mu_{b} \simeq$ $2 \mathrm{GeV}$ and $C_{2}$ denotes the coefficient of the SM charged-current operator $\mathcal{O}_{1 L L}^{s c c b}, C_{2}=$ $C_{1 L L}^{s c c b} /\left(2 \sqrt{2} G_{F} V_{L c b} V_{L c s}^{*}\right)$. We use the following values for the SM parts of these coefficients $[210]$,

$$
C_{2}^{\mathrm{SM}}(2 \mathrm{GeV})=1.204, \quad C_{7}^{\mathrm{SM}}(2 \mathrm{GeV})=-0.381, \quad C_{8}^{\mathrm{SM}}(2 \mathrm{GeV})=-0.175 .
$$

Furthermore, $\Lambda_{c} \simeq 0.38 \mathrm{GeV}$, while the three hadronic parameters, $\Lambda_{17}^{u, c}$ and $\Lambda_{78}$, are estimated to lie in the following ranges [210],

$$
\Lambda_{17}^{u} \in[-0.33,0.525] \mathrm{GeV}, \quad \Lambda_{17}^{c} \in[-0.009,0.011] \mathrm{GeV}, \quad \Lambda_{78} \in[0.017,0.19] \mathrm{GeV} .
$$

We compare the above expressions with the experimental result in table 1. 


\section{D.2.4 The $B \rightarrow K^{* 0} \gamma \mathrm{CP}$ asymmetry}

In addition we consider the time-dependent $\mathrm{CP}$ asymmetry in $B \rightarrow K^{* 0} \gamma$ decays

$$
\frac{\Gamma\left(\bar{B} \rightarrow \bar{K}^{* 0} \gamma\right)-\Gamma\left(B \rightarrow K^{* 0} \gamma\right)}{\Gamma\left(\bar{B} \rightarrow \bar{K}^{* 0} \gamma\right)+\Gamma\left(B \rightarrow K^{* 0} \gamma\right)}=S_{K^{*} \gamma} \cos \left(\Delta m_{d} t\right)+C_{K^{*} \gamma} \sin \left(\Delta m_{d} t\right)
$$

where we focus on $S_{K^{*} \gamma}$, which can be expressed as

$$
S_{K^{*} \gamma}=2 \frac{\operatorname{Im} \lambda_{K^{*} \gamma}}{1+\left|\lambda_{K^{*} \gamma}\right|^{2}}, \quad \lambda_{K^{*} \gamma}=\frac{q}{p} \frac{A\left(\bar{B} \rightarrow \bar{K}^{* 0} \gamma\right)}{A\left(B \rightarrow K^{* 0} \gamma\right)}
$$

where the ratio $\frac{q}{p}=\sqrt{\frac{M_{12}^{*}}{M_{12}}}$ arises from the phase of the $B_{d}-\bar{B}_{d}$ mixing amplitude $M_{12}$ discussed in section 5.3.1. This asymmetry is generated by the electromagnetic dipole operators, $C_{7}$ and $C_{7}^{\prime}$, at leading order and vanishes as $C_{7}^{\prime} \rightarrow 0$. The latter coefficient is suppressed by $m_{s} / m_{b}$ in the SM, while it is enhanced in the presence of $C_{H u d}$, making it a probe of right-handed currents. Using the fact that the largest BSM modifications will arise from the enhanced $C_{7}^{\prime}$ contributions we can approximate the ratio $q / p$ by its SM value, $q / p \simeq\left(V_{L t b} V_{L t d}^{*}\right) /\left(V_{L t b}^{*} V_{L t d}\right)$. The leading-order expression is then given by [204, 211],

$$
S_{K^{*} \gamma}=\frac{2 \operatorname{Im}\left(\frac{V_{L t b} V_{L t d}^{*}}{V_{L t b}^{*} V_{L t d}} \frac{V_{L t b} V_{L t s}^{*}}{V_{L t b}^{*} V_{L t s}} C_{7} C_{7}^{\prime}\right)}{\left|C_{7}\right|^{2}+\left|C_{7}^{\prime}\right|^{2}},
$$

while the SM prediction is rather small $[212,213]$

$$
S_{K^{*} \gamma}^{\mathrm{SM}}=(-2.3 \pm 1.6) \cdot 10^{-2} .
$$

The experimental value for $S_{K^{*} \gamma}$ is given in table 1 .

\section{D.2.5 Corrections to the B meson widths}

The absorptive part of the box diagrams that induce $B-\bar{B}$ oscillations give rise to the $B_{q}$ meson widths. The corrections due to $W_{L}-W_{R}$ mixing were computed in ref. [48], and are given by

$$
\begin{aligned}
\Gamma_{12}^{(q)}(\xi)=- & \frac{1}{2} \frac{G_{F}^{2} m_{b}^{2} m_{B_{q}} f_{B_{q}}^{2}}{\pi} \sqrt{z}\left(\lambda_{c}^{(q) 2}\left(\sqrt{1-4 z}-(1-z)^{2}\right)-\lambda_{c}^{(q)} \lambda_{t}^{(q)}(1-z)^{2}\right) \\
\times & {\left[\left(\left[\frac{2}{3} B_{1}-\frac{5}{6} B_{2} R\right] \frac{\xi_{c b}}{V_{L c b}}+\frac{1}{3} B_{5}\left(R+\frac{3}{2}\right) \frac{\xi_{c q}^{*}}{V_{L c q}^{*}}\right) \eta_{11 L L} \eta_{11 L R}\right.} \\
& +\left(\left[\frac{2}{3} B_{1}+\frac{1}{6} B_{3} R\right] \frac{\xi_{c b}}{V_{L c b}}+B_{4}\left(R+\frac{1}{6}\right) \frac{\xi_{c q}^{*}}{V_{L c q}^{*}}\right) \\
& \left.\times\left(\eta_{11 L L} \eta_{21 L R}+\eta_{21 L L} \eta_{11 L R}+3 \eta_{21 L L} \eta_{21 L R}\right)\right],
\end{aligned}
$$

where $z \equiv m_{c}^{2} / m_{b}^{2}, \lambda_{i}^{(q)}=V_{L i b} V_{L i q}^{*}$, and $\xi_{i j} \equiv \frac{v^{2}}{v_{R}^{2}} \frac{\xi e^{i \alpha}}{1+\xi^{2}} V_{R i j}$. The bag factors, $B_{i}$, are again given in table 2 , where the $B_{1}$ factors are related to the RG-invariant definition in 
table 2 by an RG factor, $B_{1}\left(m_{b}\right)=\hat{B}_{B_{d, s}} / 1.517$ for the $B_{d, s}$ systems [49]. The $\eta$ factors describe the RGE evolution of the four-fermion operators between $m_{W}$ and $m_{b}$, through $C_{i L L(L R)}\left(m_{b}\right)=\eta_{i j L L(L R)} C_{j L L(L R)}\left(m_{W}\right)$. Explicitly we have

$$
\begin{aligned}
\eta_{11 L L} & =\frac{1}{2}\left(\eta^{6 / 23}+\eta^{-12 / 23}\right), & \eta_{11 L R} & =\eta^{3 / 23}, \\
\eta_{21 L L} & =\frac{1}{2}\left(\eta^{6 / 23}-\eta^{-12 / 23}\right), & \eta_{21 L R} & =\frac{1}{3}\left(\eta^{-24 / 23}-\eta^{3 / 23}\right),
\end{aligned}
$$

where $\eta=\alpha_{s}\left(m_{W}\right) / \alpha_{s}\left(m_{b}\right)$.

Additional contributions arise from diagrams involving $C_{i R R}$, due to $W_{R}$ exchange

$$
\begin{aligned}
\Gamma^{(q)}\left(C_{i R R}\right)= & -\frac{1}{4} \frac{z}{2 \pi} \sqrt{1-4 z} m_{B_{q}} f_{B_{q}}^{2} m_{b}^{2}\left\{B_{4}\left(R(\mu)+\frac{1}{6}\right) C_{1 R R}^{q c c b} C_{1 L L}^{q c c b}\right. \\
& \left.+\frac{1}{3} B_{5}\left(R+\frac{3}{2}\right)\left(C_{2 R R}^{q c c b} C_{1 L L}^{q c c b}+C_{1 R R}^{q c c b} C_{2 L L}^{q c c b}+N_{c} C_{2 R R}^{q c c b} C_{2 L L}^{q c c b}\right)\right\} .
\end{aligned}
$$

The real part of these contributions to $\Gamma_{12}$ can be constrained by the width difference between the mass eigenstates, whereas $a_{\mathrm{fs}}^{q}$ is sensitive to the imaginary part [214],

$$
\Delta \Gamma^{(q)}=4 \frac{\operatorname{Re}\left(\Gamma_{12}^{(q) *} M_{12}^{(q)}\right)}{\Delta m_{q}}, \quad a_{\mathrm{fs}}^{q}=1-\left|\frac{q}{p}\right|^{2}=-\operatorname{Im}\left(\frac{\Gamma_{12}^{(q)}}{M_{12}^{(q)}}\right) .
$$

These expressions only depend on the ratio of $\Gamma_{12}^{(q)} / M_{12}^{(q)}$ and $\Delta m_{q}=2\left|M_{12}^{(q)}\right|$, which we expand in terms of the BSM contributions as follows,

$$
\frac{\Gamma_{12}^{(q)}}{M_{12}^{(q)}} \simeq \frac{\Gamma_{12}^{(q)}(\mathrm{SM})}{M_{12}^{(q)}(\mathrm{SM})}\left(1-\frac{M_{12}^{(q)}(\mathrm{LR})}{M_{12}^{(q)}(\mathrm{SM})}\right)+\frac{\Gamma_{12}^{(q)}(\mathrm{LR})}{M_{12}^{(q)}(\mathrm{SM})},
$$

where $\Gamma_{12}^{(q)}(\mathrm{LR})=\Gamma_{12}^{(q)}(\xi)+\Gamma_{12}^{(q)}\left(C_{i R R}\right)$, while $M_{12}^{(q)}(\mathrm{LR})$ is given by Eq. (5.16). We combine the mLRSM contribution with the SM prediction, which is given by [215],

$$
\left.\frac{\Gamma_{12}^{(q)}}{M_{12}^{(q)}}\right|_{\mathrm{SM}}=-10^{-4}\left[c^{(q)}+a^{(q)} \frac{\lambda_{u}^{(q)}}{\lambda_{t}^{(q)}}+b^{(q)}\left(\frac{\lambda_{u}^{(q)}}{\lambda_{t}^{(q)}}\right)^{2}\right]
$$

with

$$
\begin{array}{ll}
a^{(d)}=11.7 \pm 1.3, & a^{(s)}=12.3 \pm 1.4, \\
b^{(d)}=0.24 \pm 0.06, & b^{(s)}=0.79 \pm 0.12, \\
c^{(d)}=-49.5 \pm 8.5, & c^{(s)}=-48.0 \pm 8.3 .
\end{array}
$$

The experimental determinations are shown in table 1.

D.2.6 $\quad K_{L} \rightarrow \pi^{0} e^{+} e^{-}$

This decay is sensitive to the dipole operators $C_{\gamma d}^{d s}$ and $C_{\gamma d}^{s d}$. Due to the enhancement factors of $m_{t} / m_{s, d}$ and $m_{c} / m_{s, d}$ that appear in the matching of these Wilson coefficients, the LR model can give rise to large contributions to the branching fraction. Within 
the SM, this decay is mediated by the semi-leptonic penguin operators $C_{7 V} \bar{s} \gamma^{\mu} d \bar{e} \gamma_{\mu} e$ and $C_{7 A} \bar{s} \gamma^{\mu} d \bar{e} \gamma_{\mu} \gamma_{5} e$ [129], that give rise to direct $\mathrm{CP}$ violation. In addition, there are long-distance and indirect CPV contributions that are harder to estimate.

The above contributions involve the following vector and tensor form factors,

$$
\begin{aligned}
\left\langle\pi^{0}\left|\bar{s} \gamma^{\mu} d\right| K_{L}\right\rangle & =\frac{1}{\sqrt{2}} f_{+}^{K^{0} \pi^{+}}\left(q^{2}\right)\left(p_{K}^{\mu}+p_{\pi}^{\mu}\right), \\
\left\langle\pi^{0}\left|\bar{s} \sigma^{\mu \nu} d\right| K_{L}\right\rangle & =i f_{T}^{K \pi}\left(q^{2}\right) \frac{\sqrt{2}}{m_{K}+m_{\pi}}\left(p_{\pi}^{\mu} p_{K}^{\nu}-p_{K}^{\mu} p_{\pi}^{\nu}\right),
\end{aligned}
$$

where $f_{+}^{K \pi}$ (see table 5) is related to the vector form factor in $K^{+} \rightarrow \pi^{0} e^{+} \nu$, while $f_{T}^{K \pi}$ has been computed in ref. [216], $f_{T}^{K \pi}=0.417 \pm 0.015$, at a renormalization scale $\mu=2 \mathrm{GeV}$. This allows us to express the branching fraction as

$$
\operatorname{Br}\left(K_{L} \rightarrow \pi^{0} e^{+} e^{-}\right)=\kappa_{e}\left[\left(\operatorname{Im} \lambda_{t} \tilde{y}_{7 V}+\frac{2}{m_{K}+m_{\pi}} \frac{f_{T}^{K \pi}(0)}{f_{+}^{K \pi}(0)} 16 \pi^{2} \operatorname{Im}\left(v^{2} C_{T}\right)\right)^{2}+\operatorname{Im} \lambda_{t}^{2} \tilde{y}_{7 A}^{2}\right],
$$

where $\lambda_{t}=V_{L t s}^{*} V_{L t d}$ and $\kappa_{e}$ is introduced to cancel the SM dependence on the vector form factor $f_{+}^{K \pi}$ by normalizing to the $K^{+} \rightarrow \pi^{0} e^{+} \nu$ decay rate. $\kappa_{e}$ is defined as

$$
\begin{aligned}
\kappa_{e} & =\frac{1}{\left|V_{L u s}+\frac{v^{2}}{2} C_{H u d}^{u s}\right|^{2}} \frac{\tau\left(K_{L}\right)}{\tau\left(K^{+}\right)}\left(\frac{\alpha_{\mathrm{em}}}{2 \pi}\right)^{2} \operatorname{Br}\left(K^{+} \rightarrow \pi^{0} e^{+} \nu\right), \\
& \simeq\left(\frac{0.225}{\left|V_{L u s}+\frac{v^{2}}{2} C_{H u d}^{u s}\right|}\right)^{2} 6 \cdot 10^{-6},
\end{aligned}
$$

where we used the experimental values of ref. [119]. The BSM contributions in Eq. (D.36) arise from $C_{T}$

$$
C_{T}(\mu)=-\frac{Q_{d}}{4}\left(m_{s} C_{\gamma d}^{d s *}(\mu)+m_{d} C_{\gamma d}^{s d}(\mu)\right)
$$

while the Wilson coefficients of the SM penguin operators are given by [129]

$$
\tilde{y}_{7 V}(\mu)=P_{0}(\mu)-4\left(C_{0}\left(x_{t}\right)+\frac{1}{4} D_{0}\left(x_{t}\right)\right)+\frac{Y_{0}\left(x_{t}\right)}{s_{w}^{2}}, \quad \tilde{y}_{7 A}=-\frac{Y_{0}\left(x_{t}\right)}{s_{w}^{2}},
$$

with

$$
\begin{aligned}
Y_{0}\left(x_{t}\right) & =\frac{x_{t}}{8}\left(\frac{4-x_{t}}{1-x_{t}}+\frac{3 x_{t}}{\left(1-x_{t}\right)^{2}} \log x_{t}\right), \\
C_{0}\left(x_{t}\right) & =\frac{x_{t}}{8}\left(\frac{x_{t}-6}{x_{t}-1}+\frac{3 x_{t}+2}{\left(1-x_{t}\right)^{2}} \log x_{t}\right), \\
D_{0}\left(x_{t}\right) & =-\frac{4}{9} \log x_{t}+\frac{-19 x_{t}^{3}+25 x_{t}^{2}}{36\left(x_{t}-1\right)^{3}}+\frac{x_{t}^{2}\left(5 x_{t}^{2}-2 x_{t}-6\right)}{18\left(1-x_{t}\right)^{4}} \log x_{t},
\end{aligned}
$$

where $x_{t}=m_{t}\left(m_{W}\right)^{2} / m_{W}^{2}$ and, neglecting resummation, $P_{0}=-4 / 9 \log x_{c}$. The value of $P_{0}(\mu)$ at different scales can be found in ref. [129].

In principle, there are additional BSM contributions to Eq. (D.36) as the mLRSM can also induce the semi-leptonic penguin operators. However, these contributions are not 
enhanced by factors of $m_{t} / m_{s, d}$. In addition, the contributions from heavy Higgs exchange are suppressed by small Yukawa couplings while those from loops involving $W_{R}$ bosons have the same form as the SM contributions with $m_{W} \rightarrow M_{W_{R}}$ and $x_{t} \rightarrow m_{t}^{2} / M_{W_{R}}^{2}$ so that they are suppressed compared to the SM. It should be noted that Eq. (D.36) only contains the direct $\mathrm{CPV}$ contributions from the SM and we neglected CP-even terms and indirect contributions due to $K-\bar{K}$ mixing [129]. We nevertheless use this expression to estimate the branching ratio as the experimental limit is currently sensitive to branching ratios roughly two orders of magnitude larger than the SM prediction [119],

$$
\operatorname{BR}\left(K_{L} \rightarrow \pi^{0} e^{+} e^{-}\right)<2.8 \cdot 10^{-10} \quad(90 \% \text { C.L. }) .
$$

\section{E Renormalization group equations}

In this appendix we give several semi-analytical results for the RGE effects of the fourfermion operators discussed in section 3. As mentioned in section 3.6 the Wilson coefficients of these operators in general depend on the scale at which we integrate out the heavy LR fields. In our analysis we take this to be a single scale $\mu_{0}=M_{W_{R}}$. The resulting $\mu_{0}$ dependence of the right-handed charged currents is then approximately given by

$$
v_{R}^{2} C_{1,2 R R}^{i j k l}\left(\mu_{\mathrm{low}}\right)=\left[0.40 \eta^{2 / 7} \pm 0.79 \eta^{-4 / 7}\right]\left(V_{R}\right)_{j i}^{*}\left(V_{R}\right)_{k l},
$$

where $\eta=\frac{\alpha_{s}\left(\mu_{0}\right)}{\alpha_{s}\left(m_{t}\right)}$ and we set $\mu_{\text {low }}=2 \mathrm{GeV}$. Similar expressions can be derived for the $C_{i q u q d}$ coefficients

$$
\begin{aligned}
C_{1, q u q d}^{i j k l}\left(\mu_{\mathrm{low}}\right)= & \eta^{\frac{1+\sqrt{241}}{21}}\left[0.0045-0.093 \eta^{-6 / 7}+0.86 \eta^{-2 \sqrt{241} / 21}+2.1 \eta^{-\frac{18+2 \sqrt{241}}{21}}\right] \frac{Y_{d H}^{k l} Y_{u H}^{i j}}{M_{H}^{2}} \\
& +\eta^{\frac{1+\sqrt{241}}{21}}\left[-0.0045-0.093 \eta^{-6 / 7}-0.86 \eta^{-2 \sqrt{241} / 21}+2.1 \eta^{-\frac{18+2 \sqrt{241}}{21}}\right] \frac{Y_{d H}^{i l} Y_{u H}^{k j}}{M_{H}^{2}}, \\
C_{2 q u q d}^{i j k l}\left(\mu_{\mathrm{low}}\right)= & \eta^{\frac{1+\sqrt{241}}{21}}\left[0.017+0.17 \eta^{-6 / 7}-0.056 \eta^{-2 \sqrt{241} / 21}-0.57 \eta^{-\frac{18+2 \sqrt{241}}{21}}\right] \frac{Y_{d H}^{k l} Y_{u H}^{i j}}{M_{H}^{2}} \\
& \eta^{\frac{1+\sqrt{241}}{21}}\left[-0.017+0.17 \eta^{-6 / 7}+0.056 \eta^{-2 \sqrt{241} / 21}-0.57 \eta^{-\frac{18+2 \sqrt{241}}{21}}\right] \frac{Y_{d H}^{i l} Y_{u H}^{k j}}{M_{H}^{2}},
\end{aligned}
$$

where $Y_{q H}$ are to be evaluated at $\mu=\mu_{0}$. Finally, the Wilson coefficients for the $\Delta F=2$ operators can be written as

$$
\begin{aligned}
& C_{4}^{i j k l}\left(\mu_{\text {low }}\right)=\frac{g_{R}^{2}}{M_{W_{R}}^{2}} \sum_{a, b} a_{a b}^{(4)} \frac{m_{u_{a}} m_{u_{b}}}{m_{t}^{2}} V_{L a i}^{*} V_{L b j}\left(V_{R}\right)_{b k}^{*}\left(V_{R}\right)_{a l}, \\
& C_{5}^{i j k l}\left(\mu_{\text {low }}\right)=-1.26 \eta^{-8 / 7} \frac{1}{M_{H}^{2}}\left(Y_{d H}\right)_{j k}^{*} Y_{d H}^{i l}+\frac{g_{R}^{2}}{M_{W_{R}}^{2}} \sum_{a, b} a_{a b}^{(5)} \frac{m_{u_{a}} m_{u_{b}}}{m_{t}^{2}} V_{L a i}^{*} V_{L b j}\left(V_{R}\right)_{b k}^{*}\left(V_{R}\right)_{a l},
\end{aligned}
$$

with $Y_{q H}$ again evaluated at $\mu=\mu_{0}$, while the coefficients $a^{(4,5)}$ are now functions of $\mu_{0}$ and are given by,

$$
\begin{aligned}
& a^{(4)}=-0.024\left[a_{1}^{(4)}+a_{2}^{(4)} \eta^{-6 / 7}+a_{3}^{(4)} \ln \eta\right] \eta^{2 / 7} \\
& a^{(5)}=-0.024\left[a_{1}^{(5)} \eta^{1 / 7}+a_{2}^{(5)} \eta^{-2 / 7}+a_{3}^{(5)} \eta+a_{4}^{(5)} \eta \ln \eta\right] \eta^{-5 / 7}
\end{aligned}
$$


where the coefficients for $a^{(4)}$ are

$$
a_{1}^{(4)}=\left(\begin{array}{ccc}
1 & 1 & 0.53 \\
1 & 0.97 & 0.55 \\
0.53 & 0.55 & 0.50
\end{array}\right), \quad a_{2}^{(4)}=-\left(\begin{array}{ccc}
1.75 & 1.75 & 0.53 \\
1.75 & 1.86 & 0.55 \\
0.53 & 0.55 & 0.50
\end{array}\right), \quad a_{3}^{(4)}=-0.42\left(\begin{array}{lll}
1 & 1 & 1 \\
1 & 1 & 1 \\
1 & 1 & 1
\end{array}\right),
$$

while those for $a^{(5)}$ are

$$
\begin{array}{ll}
a_{1}^{(5)}=\left(\begin{array}{ccc}
1 & 1 & -1.58 \\
1 & 1.22 & -1.53 \\
-1.58 & -1.53 & -1.64
\end{array}\right), \quad a_{2}^{(5)}=3.03\left(\begin{array}{lll}
1 & 1 & 1 \\
1 & 1 & 1 \\
1 & 1 & 1
\end{array}\right), \\
a_{3}^{(5)}=\left(\begin{array}{ccc}
0.90 & 0.90 & -1.25 \\
0.90 & 0.97 & -1.19 \\
-1.25 & -1.19 & -1.34
\end{array}\right), \quad a_{4}^{(5)}=0.14\left(\begin{array}{lll}
1 & 1 & 1 \\
1 & 1 & 1 \\
1 & 1 & 1
\end{array}\right) .
\end{array}
$$

The terms $\sim \log \eta$ arise from the fact that the anomalous dimension matrix in Eq. (3.9) has degenerate eigenvalues at $n_{f}=6$, leading to contributions of the form $\sim \frac{\eta^{\epsilon}-1}{\epsilon}$ with $\epsilon \propto n_{f}-6$.

Open Access. This article is distributed under the terms of the Creative Commons Attribution License (CC-BY 4.0), which permits any use, distribution and reproduction in any medium, provided the original author(s) and source are credited.

\section{References}

[1] E.E. Jenkins, A.V. Manohar and P. Stoffer, Low-Energy Effective Field Theory below the Electroweak Scale: Operators and Matching, JHEP 03 (2018) 016 [arXiv:1709.04486] [INSPIRE].

[2] J.C. Pati and A. Salam, Lepton Number as the Fourth Color, Phys. Rev. D 10 (1974) 275 [Erratum ibid. 11 (1975) 703] [INSPIRE].

[3] R.N. Mohapatra and J.C. Pati, Left-Right Gauge Symmetry and an Isoconjugate Model of CP-violation, Phys. Rev. D 11 (1975) 566 [InSPIRE].

[4] G. Senjanović and R.N. Mohapatra, Exact Left-Right Symmetry and Spontaneous Violation of Parity, Phys. Rev. D 12 (1975) 1502 [inSPIRE].

[5] G. Senjanović, Spontaneous Breakdown of Parity in a Class of Gauge Theories, Nucl. Phys. $B 153$ (1979) 334 [INSPIRE].

[6] N.G. Deshpande, J.F. Gunion, B. Kayser and F.I. Olness, Left-right symmetric electroweak models with triplet Higgs, Phys. Rev. D 44 (1991) 837 [INSPIRE].

[7] R.N. Mohapatra and G. Senjanović, Neutrino Mass and Spontaneous Parity Nonconservation, Phys. Rev. Lett. 44 (1980) 912 [inSPIRE].

[8] R.N. Mohapatra and G. Senjanović, Neutrino Masses and Mixings in Gauge Models with Spontaneous Parity Violation, Phys. Rev. D 23 (1981) 165 [InSPIRE].

[9] M. Doi, T. Kotani and E. Takasugi, Double beta Decay and Majorana Neutrino, Prog. Theor. Phys. Suppl. 83 (1985) 1 [inSPIRE]. 
[10] V. Tello, M. Nemevšek, F. Nesti, G. Senjanović and F. Vissani, Left-Right Symmetry: from LHC to Neutrinoless Double Beta Decay, Phys. Rev. Lett. 106 (2011) 151801 [arXiv:1011.3522] [INSPIRE].

[11] W. Rodejohann, Neutrino-less Double Beta Decay and Particle Physics, Int. J. Mod. Phys. E 20 (2011) 1833 [arXiv:1106.1334] [INSPIRE].

[12] V. Cirigliano, W. Dekens, J. de Vries, M.L. Graesser and E. Mereghetti, A neutrinoless double beta decay master formula from effective field theory, JHEP 12 (2018) 097 [arXiv: 1806.02780] [INSPIRE].

[13] G. Li, M. Ramsey-Musolf and J.C. Vasquez, Left-Right Symmetry and Leading Contributions to Neutrinoless Double Beta Decay, Phys. Rev. Lett. 126 (2021) 151801 [arXiv:2009.01257] [INSPIRE].

[14] J.L. Yang, C.-H. Chang and T.-F. Feng, Nuclear $0 \nu 2 \beta$ decays in B - L symmetric SUSY model and TeV scale left-right symmetric model, arXiv:2107.01367 [INSPIRE].

[15] W.-Y. Keung and G. Senjanović, Majorana Neutrinos and the Production of the Right-handed Charged Gauge Boson, Phys. Rev. Lett. 50 (1983) 1427 [INSPIRE].

[16] G. Beall, M. Bander and A. Soni, Constraint on the Mass Scale of a Left-Right Symmetric Electroweak Theory from the $K_{L}-K_{S}$ Mass Difference, Phys. Rev. Lett. 48 (1982) 848 [INSPIRE].

[17] R.N. Mohapatra, G. Senjanović and M.D. Tran, Strangeness Changing Processes and the Limit on the Right-handed Gauge Boson Mass, Phys. Rev. D 28 (1983) 546 [InSPIRE].

[18] G. Ecker, W. Grimus and H. Neufeld, Higgs Induced Flavor Changing Neutral Interactions in $\mathrm{SU}(2)_{L} \times \mathrm{SU}(2)_{R} \times \mathrm{U}(1)$, Phys. Lett. B 127 (1983) 365 [Erratum ibid. 132 (1983) 467] [INSPIRE].

[19] G. Ecker and W. Grimus, Mass Mixing, CP Violation and Left-right Symmetry for Heavy Neutral Mesons, Z. Phys. C 30 (1986) 293 [inSPIRE].

[20] J.M. Frère, J. Galand, A. Le Yaouanc, L. Oliver, O. Pene and J.C. Raynal, $K^{0} \bar{K}^{0}$ in the $\mathrm{SU}(2)_{L} \times \mathrm{SU}(2)_{R} \times \mathrm{U}(1)$ model of CP-violation, Phys. Rev. D 46 (1992) 337 [InSPIRE].

[21] P. Ball, J.M. Frère and J. Matias, Anatomy of mixing induced CP asymmetries in left-right symmetric models with spontaneous CP-violation, Nucl. Phys. B 572 (2000) 3 [hep-ph/9910211] [INSPIRE].

[22] S. Bertolini, A. Maiezza and F. Nesti, Present and Future $K$ and B Meson Mixing Constraints on TeV Scale Left-Right Symmetry, Phys. Rev. D 89 (2014) 095028 [arXiv:1403.7112] [INSPIRE].

[23] M. González-Alonso, O. Naviliat-Cuncic and N. Severijns, New physics searches in nuclear and neutron $\beta$ decay, Prog. Part. Nucl. Phys. 104 (2019) 165 [arXiv:1803.08732] [InSPIRE].

[24] V. Cirigliano, S. Gardner and B. Holstein, Beta Decays and Non-Standard Interactions in the LHC Era, Prog. Part. Nucl. Phys. 71 (2013) 93 [arXiv:1303.6953] [INSPIRE].

[25] K. Hsieh, K. Schmitz, J.-H. Yu and C.P. Yuan, Global Analysis of General $\mathrm{SU}(2) \times \mathrm{SU}(2) \times \mathrm{U}(1)$ Models with Precision Data, Phys. Rev. D 82 (2010) 035011 [arXiv: 1003.3482] [INSPIRE].

[26] M. Blanke, A.J. Buras, K. Gemmler and T. Heidsieck, $\Delta F=2$ observables and $B \rightarrow X_{q} \gamma$ decays in the Left-Right Model: Higgs particles striking back, JHEP 03 (2012) 024 [arXiv:1111.5014] [INSPIRE]. 
[27] V. Bernard, S. Descotes-Genon and L. Vale Silva, Constraining the gauge and scalar sectors of the doublet left-right symmetric model, JHEP 09 (2020) 088 [arXiv:2001.00886] [INSPIRE].

[28] G. Ecker, W. Grimus and H. Neufeld, The Neutron Electric Dipole Moment in Left-right Symmetric Gauge Models, Nucl. Phys. B 229 (1983) 421 [inSPIRE].

[29] J.M. Frère, J. Galand, A. Le Yaouanc, L. Oliver, O. Pene and J.C. Raynal, The neutron electric dipole moment in left-right symmetric models, Phys. Rev. D 45 (1992) 259 [INSPIRE].

[30] F. Xu, H. An and X. Ji, Neutron Electric Dipole Moment Constraint on Scale of Minimal Left-Right Symmetric Model, JHEP 03 (2010) 088 [arXiv:0910.2265] [INSPIRE].

[31] V. Cirigliano, W. Dekens, J. de Vries and E. Mereghetti, An $\epsilon^{\prime}$ improvement from right-handed currents, Phys. Lett. B 767 (2017) 1 [arXiv:1612.03914] [INSPIRE].

[32] CMS collaboration, Search for narrow and broad dijet resonances in proton-proton collisions at $\sqrt{s}=13 \mathrm{TeV}$ and constraints on dark matter mediators and other new particles, JHEP 08 (2018) 130 [arXiv:1806.00843] [INSPIRE].

[33] ATLAS collaboration, Search for new phenomena in dijet events using $37 \mathrm{fb}^{-1}$ of pp collision data collected at $\sqrt{s}=13 \mathrm{TeV}$ with the ATLAS detector, Phys. Rev. D 96 (2017) 052004 [arXiv: 1703.09127] [INSPIRE].

[34] CMS collaboration, Search for $W^{\prime}$ bosons decaying to a top and a bottom quark at $\sqrt{s}=13 \mathrm{TeV}$ in the hadronic final state, Phys. Lett. B $\mathbf{8 2 0}$ (2021) 136535 [arXiv:2104.04831] [INSPIRE].

[35] H.-L. Li, Z. Ren, M.-L. Xiao, J.-H. Yu and Y.-H. Zheng, Operator bases in effective field theories with sterile neutrinos: $d \leq 9$, JHEP 11 (2021) 003 [arXiv:2105.09329] [INSPIRE].

[36] Y. Liao and X.-D. Ma, Operators up to Dimension Seven in Standard Model Effective Field Theory Extended with Sterile Neutrinos, Phys. Rev. D 96 (2017) 015012 [arXiv:1612.04527] [INSPIRE].

[37] F. del Aguila, S. Bar-Shalom, A. Soni and J. Wudka, Heavy Majorana Neutrinos in the Effective Lagrangian Description: Application to Hadron Colliders, Phys. Lett. B 670 (2009) 399 [arXiv:0806.0876] [INSPIRE].

[38] Y. Zhang, H. An, X. Ji and R.N. Mohapatra, General CP-violation in Minimal Left-Right Symmetric Model and Constraints on the Right-Handed Scale, Nucl. Phys. B 802 (2008) 247 [arXiv:0712.4218] [INSPIRE].

[39] S. Bertolini, A. Maiezza and F. Nesti, Kaon CP-violation and neutron EDM in the minimal left-right symmetric model, Phys. Rev. D 101 (2020) 035036 [arXiv:1911.09472] [INSPIRE].

[40] A. Maiezza and M. Nemevšek, Strong P invariance, neutron electric dipole moment, and minimal left-right parity at LHC, Phys. Rev. D 90 (2014) 095002 [arXiv:1407.3678] [INSPIRE].

[41] A. Maiezza, M. Nemevšek, F. Nesti and G. Senjanović, Left-Right Symmetry at LHC, Phys. Rev. D 82 (2010) 055022 [arXiv: 1005.5160] [INSPIRE].

[42] G. Prézeau, M. Ramsey-Musolf and P. Vogel, Neutrinoless double beta decay and effective field theory, Phys. Rev. D 68 (2003) 034016 [hep-ph/0303205] [INSPIRE].

[43] G. Bambhaniya, P.S.B. Dev, S. Goswami and M. Mitra, The Scalar Triplet Contribution to Lepton Flavour Violation and Neutrinoless Double Beta Decay in Left-Right Symmetric Model, JHEP 04 (2016) 046 [arXiv:1512.00440] [INSPIRE]. 
[44] P.S. Bhupal Dev, S. Goswami and M. Mitra, TeV Scale Left-Right Symmetry and Large Mixing Effects in Neutrinoless Double Beta Decay, Phys. Rev. D 91 (2015) 113004 [arXiv: 1405.1399] [INSPIRE].

[45] M. Nemevšek, F. Nesti, G. Senjanović and V. Tello, Neutrinoless Double Beta Decay: Low Left-Right Symmetry Scale?, arXiv:1112.3061 [INSPIRE].

[46] M. Nemevšek, G. Senjanović and V. Tello, Connecting Dirac and Majorana Neutrino Mass Matrices in the Minimal Left-Right Symmetric Model, Phys. Rev. Lett. 110 (2013) 151802 [arXiv: 1211.2837] [INSPIRE].

[47] J. Barry and W. Rodejohann, Lepton number and flavour violation in TeV-scale left-right symmetric theories with large left-right mixing, JHEP 09 (2013) 153 [arXiv:1303.6324] [INSPIRE].

[48] S. Alioli, V. Cirigliano, W. Dekens, J. de Vries and E. Mereghetti, Right-handed charged currents in the era of the Large Hadron Collider, JHEP 05 (2017) 086 [arXiv:1703.04751] [INSPIRE].

[49] Flavour Lattice Averaging Group collaboration, FLAG Review 2019: Flavour Lattice Averaging Group (FLAG), Eur. Phys. J. C 80 (2020) 113 [arXiv:1902.08191] [InSPIRE].

[50] RBC and UKQCD collaborations, Direct CP-violation and the $\Delta I=1 / 2$ rule in $K \rightarrow \pi \pi$ decay from the standard model, Phys. Rev. D 102 (2020) 054509 [arXiv:2004.09440] [INSPIRE].

[51] J. Brod, M. Gorbahn and E. Stamou, Standard-Model Prediction of $\epsilon_{K}$ with Manifest Quark-Mixing Unitarity, Phys. Rev. Lett. 125 (2020) 171803 [arXiv:1911.06822] [INSPIRE].

[52] Q.-H. Cao, Z. Li, J.-H. Yu and C.P. Yuan, Discovery and Identification of $W^{\prime}$ and $Z$ ' in $\mathrm{SU}(2) \times \mathrm{SU}(2) \times \mathrm{U}(1)$ Models at the LHC, Phys. Rev. D 86 (2012) 095010 [arXiv: 1205.3769] [INSPIRE].

[53] J. Harz, M.J. Ramsey-Musolf, T. Shen and S. Urrutia-Quiroga, TeV-scale Lepton Number Violation: Connecting Leptogenesis, Neutrinoless Double Beta Decay, and Colliders, arXiv:2106.10838 [INSPIRE].

[54] W. Dekens and D. Boer, Viability of minimal left-right models with discrete symmetries, Nucl. Phys. B 889 (2014) 727 [arXiv: 1409.4052] [INSPIRE].

[55] P.S. Bhupal Dev, R.N. Mohapatra, W. Rodejohann and X.-J. Xu, Vacuum structure of the left-right symmetric model, JHEP 02 (2019) 154 [arXiv: 1811.06869] [INSPIRE].

[56] G. Chauhan, Vacuum Stability and Symmetry Breaking in Left-Right Symmetric Model, JHEP 12 (2019) 137 [arXiv:1907.07153] [INSPIRE].

[57] A. Maiezza, M. Nemevšek and F. Nesti, Perturbativity and mass scales in the minimal left-right symmetric model, Phys. Rev. D 94 (2016) 035008 [arXiv:1603.00360] [INSPIRE].

[58] J. Chakrabortty, P. Konar and T. Mondal, Copositive Criteria and Boundedness of the Scalar Potential, Phys. Rev. D 89 (2014) 095008 [arXiv:1311.5666] [INSPIRE].

[59] G.C. Branco, J.M. Frère and J.M. Gerard, The Value of $\epsilon^{\prime} / \epsilon$ in Models Based on $\mathrm{SU}(2)_{L} \times \mathrm{SU}(2)_{R} \times \mathrm{U}(1)$, Nucl. Phys. B 221 (1983) 317 [InSPIRE].

[60] G. Senjanović and V. Tello, Right Handed Quark Mixing in Left-Right Symmetric Theory, Phys. Rev. Lett. 114 (2015) 071801 [arXiv: 1408.3835] [INSPIRE].

[61] G. Senjanović and V. Tello, Restoration of Parity and the Right-Handed Analog of the CKM Matrix, Phys. Rev. D 94 (2016) 095023 [arXiv: 1502.05704] [INSPIRE]. 
[62] NEDM collaboration, Measurement of the permanent electric dipole moment of the neutron, Phys. Rev. Lett. 124 (2020) 081803 [arXiv:2001.11966] [InSPIRE].

[63] J. Dragos, T. Luu, A. Shindler, J. de Vries and A. Yousif, Confirming the Existence of the strong CP Problem in Lattice QCD with the Gradient Flow, Phys. Rev. C 103 (2021) 015202 [arXiv: 1902.03254] [INSPIRE].

[64] G. Senjanović and V. Tello, Strong CP-violation: problem or blessing?, arXiv: 2004.04036 [INSPIRE].

[65] L. Ubaldi, Effects of theta on the deuteron binding energy and the triple-alpha process, Phys. Rev. D 81 (2010) 025011 [arXiv:0811.1599] [INSPIRE].

[66] D. Lee, U.-G. Meißner, K.A. Olive, M. Shifman and T. Vonk, $\theta$-dependence of light nuclei and nucleosynthesis, Phys. Rev. Res. 2 (2020) 033392 [arXiv:2006.12321] [INSPIRE].

[67] J. de Vries, P. Draper, K. Fuyuto, J. Kozaczuk and B. Lillard, Uncovering an axion mechanism with the EDM portfolio, Phys. Rev. D 104 (2021) 055039 [arXiv:2107.04046] [INSPIRE].

[68] J.E. Moody and F. Wilczek, New macroscopic forces?, Phys. Rev. D 30 (1984) 130 [inSPIRE].

[69] G. Raffelt, Limits on a CP-violating scalar axion-nucleon interaction, Phys. Rev. D 86 (2012) 015001 [arXiv: 1205.1776] [INSPIRE].

[70] S. Bertolini, L. Di Luzio and F. Nesti, Axion-mediated forces, CP-violation and left-right interactions, Phys. Rev. Lett. 126 (2021) 081801 [arXiv: 2006.12508] [INSPIRE].

[71] C.A.J. O'Hare and E. Vitagliano, Cornering the axion with CP-violating interactions, Phys. Rev. D 102 (2020) 115026 [arXiv: 2010.03889] [INSPIRE].

[72] B. Grzadkowski, M. Iskrzyński, M. Misiak and J. Rosiek, Dimension-Six Terms in the Standard Model Lagrangian, JHEP 10 (2010) 085 [arXiv:1008.4884] [INSPIRE].

[73] D. Chang, J. Basecq, L.-F. Li and P.B. Pal, Comment on the $K_{L} K_{S}$ Mass Difference in Left-right Model, Phys. Rev. D 30 (1984) 1601 [inSPIRE].

[74] J. Basecq, L.-F. Li and P.B. Pal, Gauge Invariant Calculation of the $K_{L} K_{S}$ Mass Difference in the Left-right Model, Phys. Rev. D 32 (1985) 175 [INSPIRE].

[75] G. Ecker and W. Grimus, CP Violation and Left-Right Symmetry, Nucl. Phys. B 258 (1985) 328 [INSPIRE].

[76] A.J. Buras and P.H. Weisz, QCD Nonleading Corrections to Weak Decays in Dimensional Regularization and 't Hooft-Veltman Schemes, Nucl. Phys. B 333 (1990) 66 [inSPIRE].

[77] M.J. Dugan and B. Grinstein, On the vanishing of evanescent operators, Phys. Lett. B 256 (1991) 239 [INSPIRE].

[78] S. Herrlich and U. Nierste, Evanescent operators, scheme dependences and double insertions, Nucl. Phys. B 455 (1995) 39 [hep-ph/9412375] [INSPIRE].

[79] W. Dekens and P. Stoffer, Low-energy effective field theory below the electroweak scale: matching at one loop, JHEP 10 (2019) 197 [arXiv:1908.05295] [INSPIRE].

[80] A.J. Buras, S. Jäger and J. Urban, Master formulae for Delta F=2 NLO QCD factors in the standard model and beyond, Nucl. Phys. B 605 (2001) 600 [hep-ph/0102316] [INSPIRE].

[81] R. Alonso, E.E. Jenkins, A.V. Manohar and M. Trott, Renormalization Group Evolution of the Standard Model Dimension Six Operators III: Gauge Coupling Dependence and Phenomenology, JHEP 04 (2014) 159 [arXiv:1312.2014] [INSPIRE]. 
[82] G. Degrassi, E. Franco, S. Marchetti and L. Silvestrini, QCD corrections to the electric dipole moment of the neutron in the MSSM, JHEP 11 (2005) 044 [hep-ph/0510137] [INSPIRE].

[83] W. Dekens and J. de Vries, Renormalization Group Running of Dimension-Six Sources of Parity and Time-Reversal Violation, JHEP 05 (2013) 149 [arXiv:1303.3156] [INSPIRE].

[84] J. Hisano, J.Y. Lee, N. Nagata and Y. Shimizu, Reevaluation of Neutron Electric Dipole Moment with QCD Sum Rules, Phys. Rev. D 85 (2012) 114044 [arXiv:1204.2653] [InSPIRE].

[85] E. Braaten, C.-S. Li and T.-C. Yuan, The Evolution of Weinberg's Gluonic CP Violation Operator, Phys. Rev. Lett. 64 (1990) 1709 [inSPIRE].

[86] G. Boyd, A.K. Gupta, S.P. Trivedi and M.B. Wise, Effective Hamiltonian for the Electric Dipole Moment of the Neutron, Phys. Lett. B 241 (1990) 584 [INSPIRE].

[87] P.L. Cho and M. Misiak, $b \rightarrow$ s $\gamma$ decay in $\mathrm{SU}(2)_{L} \times \mathrm{SU}(2)_{R} \times \mathrm{U}(1)$ extensions of the Standard Model, Phys. Rev. D 49 (1994) 5894 [hep-ph/9310332] [InSPIRE].

[88] F. Wilczek and A. Zee, $\Delta I=\frac{1}{2}$ Rule and Right-Handed Currents: Heavy Quark Expansion and Limitation on Zweig's Rule, Phys. Rev. D 15 (1977) 2660 [InSPIRE].

[89] S. Weinberg, Larger Higgs Exchange Terms in the Neutron Electric Dipole Moment, Phys. Rev. Lett. 63 (1989) 2333 [INSPIRE].

[90] M.I. Vysotsky, $K^{0} \bar{K}^{0}$ transition in the standard $\mathrm{SU}(3) \times \mathrm{SU}(2) \times \mathrm{U}(1)$ model, Sov. J. Nucl. Phys. 31 (1980) 797 [INSPIRE].

[91] RBC and UKQCD collaborations, Standard Model Prediction for Direct CP-violation in $K \rightarrow \pi \pi$ Decay, Phys. Rev. Lett. 115 (2015) 212001 [arXiv:1505. 07863] [InSPIRE].

[92] T. Blum et al., Lattice determination of the $K \rightarrow(\pi \pi)_{I=2}$ Decay Amplitude $A_{2}$, Phys. Rev. D 86 (2012) 074513 [arXiv: 1206.5142] [INSPIRE].

[93] A. Nicholson et al., Heavy physics contributions to neutrinoless double beta decay from QCD, Phys. Rev. Lett. 121 (2018) 172501 [arXiv:1805.02634] [INSPIRE].

[94] V. Cirigliano, W. Dekens, M. Graesser and E. Mereghetti, Neutrinoless double beta decay and chiral SU(3), Phys. Lett. B $\mathbf{7 6 9}$ (2017) 460 [arXiv:1701.01443] [INSPIRE].

[95] J. de Vries, E. Mereghetti, R.G.E. Timmermans and U. van Kolck, The Effective Chiral Lagrangian From Dimension-Six Parity and Time-Reversal Violation, Annals Phys. 338 (2013) 50 [arXiv: 1212.0990] [INSPIRE].

[96] E. Mereghetti, W.H. Hockings and U. van Kolck, The Effective Chiral Lagrangian From the Theta Term, Annals Phys. 325 (2010) 2363 [arXiv:1002.2391] [INSPIRE].

[97] J. Bsaisou, U.-G. Meißner, A. Nogga and A. Wirzba, P- and T-Violating Lagrangians in Chiral Effective Field Theory and Nuclear Electric Dipole Moments, Annals Phys. 359 (2015) 317 [arXiv: 1412.5471] [InSPIRE].

[98] N. Haba, H. Umeeda and T. Yamada, $\epsilon^{\prime} / \epsilon$ Anomaly and Neutron EDM in $\mathrm{SU}(2)_{L} \times \mathrm{SU}(2)_{R} \times \mathrm{U}(1)_{B-L}$ model with Charge Symmetry, JHEP 05 (2018) 052 [arXiv: 1802.09903] [INSPIRE].

[99] J. de Vries, E. Mereghetti, C.-Y. Seng and A. Walker-Loud, Lattice QCD spectroscopy for hadronic CP-violation, Phys. Lett. B $\mathbf{7 6 6}$ (2017) 254 [arXiv: 1612.01567] [INSPIRE].

[100] C.-Y. Seng and M. Ramsey-Musolf, Parity-violating and time-reversal-violating pion-nucleon couplings: Higher order chiral matching relations, Phys. Rev. C 96 (2017) 065204 [arXiv: 1611.08063] [INSPIRE]. 
[101] M. Hoferichter, J. Ruiz de Elvira, B. Kubis and U.-G. Meißner, High-Precision Determination of the Pion-Nucleon $\sigma$ Term from Roy-Steiner Equations, Phys. Rev. Lett. 115 (2015) 092301 [arXiv: 1506.04142] [INSPIRE].

[102] S. Aoki et al., Review of lattice results concerning low-energy particle physics, Eur. Phys. J. C 77 (2017) 112 [arXiv: 1607.00299] [INSPIRE].

[103] Budapest-Marseille-Wuppertal collaboration, Isospin splittings in the light baryon octet from lattice QCD and QED, Phys. Rev. Lett. 111 (2013) 252001 [arXiv:1306.2287] [INSPIRE].

[104] S. Borsányi et al., Ab initio calculation of the neutron-proton mass difference, Science $\mathbf{3 4 7}$ (2015) 1452 [arXiv: 1406 .4088] [INSPIRE].

[105] M. Pospelov, Best values for the CP odd meson nucleon couplings from supersymmetry, Phys. Lett. B 530 (2002) 123 [hep-ph/0109044] [INSPIRE].

[106] J.C. Hardy and I.S. Towner, Superallowed $0^{+} \rightarrow 0^{+}$nuclear $\beta$ decays: 2020 critical survey, with implications for $V_{u d}$ and CKM unitarity, Phys. Rev. C 102 (2020) 045501 [INSPIRE].

[107] ATLAS collaboration, Study of the rare decays of $B_{s}^{0}$ and $B^{0}$ mesons into muon pairs using data collected during 2015 and 2016 with the ATLAS detector, JHEP 04 (2019) 098 [arXiv: 1812.03017] [INSPIRE].

[108] KOTO collaboration, Study of the $K_{L} \rightarrow \pi^{0} \nu \bar{\nu}$ Decay at the J-PARC KOTO Experiment, Phys. Rev. Lett. 126 (2021) 121801 [arXiv: 2012.07571] [InSPIRE].

[109] NA62 collaboration, Measurement of the very rare $K^{+} \rightarrow \pi^{+} \nu \bar{\nu}$ decay, JHEP 06 (2021) 093 [arXiv:2103.15389] [INSPIRE].

[110] C.-Y. Seng, Reexamination of The Standard Model Nucleon Electric Dipole Moment, Phys. Rev. C 91 (2015) 025502 [arXiv:1411.1476] [InSPIRE].

[111] M. Pospelov and A. Ritz, Electric dipole moments as probes of new physics, Annals Phys. 318 (2005) 119 [hep-ph/0504231] [INSPIRE].

[112] A. Czarnecki and B. Krause, Neutron electric dipole moment in the standard model: Valence quark contributions, Phys. Rev. Lett. 78 (1997) 4339 [hep-ph/9704355] [INSPIRE].

[113] T. Mannel and N. Uraltsev, Loop-Less Electric Dipole Moment of the Nucleon in the Standard Model, Phys. Rev. D 85 (2012) 096002 [arXiv:1202.6270] [InSPIRE].

[114] C.-Y. Seng, M. Gorchtein, H.H. Patel and M.J. Ramsey-Musolf, Reduced Hadronic Uncertainty in the Determination of $V_{u d}$, Phys. Rev. Lett. 121 (2018) 241804 [arXiv: 1807.10197] [INSPIRE].

[115] A. Czarnecki, W.J. Marciano and A. Sirlin, Radiative Corrections to Neutron and Nuclear Beta Decays Revisited, Phys. Rev. D 100 (2019) 073008 [arXiv: 1907.06737] [InSPIRE].

[116] C.Y. Seng, M. Gorchtein and M.J. Ramsey-Musolf, Dispersive evaluation of the inner radiative correction in neutron and nuclear $\beta$ decay, Phys. Rev. D 100 (2019) 013001 [arXiv: 1812.03352] [INSPIRE].

[117] J.C. Hardy and I.S. Towner, Superallowed $0^{+} \rightarrow 0^{+}$nuclear $\beta$ decays: 2014 critical survey, with precise results for $V_{u d}$ and CKM unitarity, Phys. Rev. C 91 (2015) 025501 [arXiv:1411.5987] [INSPIRE].

[118] J. Hardy and I.S. Towner, $\left|V_{u d}\right|$ from nuclear $\beta$ decays, PoS CKM2016 (2016) 028 [INSPIRE]. 
[119] Particle Data Group collaboration, Review of Particle Physics, PTEP 2020 (2020) 083C01 [INSPIRE].

[120] T. Bhattacharya et al., Probing Novel Scalar and Tensor Interactions from (Ultra)Cold Neutrons to the LHC, Phys. Rev. D 85 (2012) 054512 [arXiv:1110.6448] [INSPIRE].

[121] V. Bernard, M. Oertel, E. Passemar and J. Stern, Tests of non-standard electroweak couplings of right-handed quarks, JHEP 01 (2008) 015 [arXiv:0707.4194] [INSPIRE].

[122] C.C. Chang et al., A per-cent-level determination of the nucleon axial coupling from quantum chromodynamics, Nature 558 (2018) 91 [arXiv: 1805.12130] [INSPIRE].

[123] Flavianet Working Group on Kaon Decays collaboration, An evaluation of $\left|V_{u s}\right|$ and precise tests of the Standard Model from world data on leptonic and semileptonic kaon decays, Eur. Phys. J. C 69 (2010) 399 [arXiv: 1005.2323] [InSPIRE].

[124] Particle Data Group collaboration, Review of Particle Physics, Phys. Rev. D 98 (2018) 030001 [INSPIRE].

[125] Fermilab Lattice and MILC collaborations, $\left|V_{u s}\right|$ from $K_{\ell 3}$ decay and four-flavor lattice QCD, Phys. Rev. D 99 (2019) 114509 [arXiv:1809.02827] [InSPIRE].

[126] J.D. Jackson, S.B. Treiman and H.W. Wyld, Possible tests of time reversal invariance in Beta decay, Phys. Rev. 106 (1957) 517 [inSPIRE].

[127] H.P. Mumm et al., A New Limit on Time-Reversal Violation in Beta Decay, Phys. Rev. Lett. 107 (2011) 102301 [arXiv: 1104.2778] [InSPIRE].

[128] S.Y. Hsueh et al., A High Precision Measurement of Polarized Sigma- beta Decay, Phys. Rev. D 38 (1988) 2056 [INSPIRE].

[129] G. Buchalla, A.J. Buras and M.E. Lautenbacher, Weak decays beyond leading logarithms, Rev. Mod. Phys. 68 (1996) 1125 [hep-ph/9512380] [INSPIRE].

[130] A.J. Buras, Flavor physics and CP-violation, in 2004 European School of High-Energy Physics, (2005), pp. 95-168 [hep-ph/0505175] [INSPIRE].

[131] V. Cirigliano, G. Ecker, H. Neufeld, A. Pich and J. Portoles, Kaon Decays in the Standard Model, Rev. Mod. Phys. 84 (2012) 399 [arXiv:1107.6001] [InSPIRE].

[132] V. Cirigliano, H. Gisbert, A. Pich and A. Rodríguez-Sánchez, Isospin-violating contributions to $\epsilon^{\prime} / \epsilon$, JHEP 02 (2020) 032 [arXiv: 1911.01359] [INSPIRE].

[133] HFLAV collaboration, Averages of b-hadron, c-hadron, and $\tau$-lepton properties as of 2018, Eur. Phys. J. C 81 (2021) 226 [arXiv:1909.12524] [inSPIRE].

[134] LHCb collaboration, Precise determination of the $B_{s}^{0}-\bar{B}_{s}^{0}$ oscillation frequency, arXiv:2104.04421 [INSPIRE].

[135] A.J. Buras and J. Girrbach, Towards the Identification of New Physics through Quark Flavour Violating Processes, Rept. Prog. Phys. 77 (2014) 086201 [arXiv:1306.3775] [InSPIRE].

[136] Fermilab Lattice and MILC collaborations, $B_{(s)}^{0}$-mixing matrix elements from lattice QCD for the Standard Model and beyond, Phys. Rev. D 93 (2016) 113016 [arXiv: 1602.03560] [INSPIRE].

[137] A.J. Buras, Weak Hamiltonian, CP-violation and rare decays, in Les Houches Summer School in Theoretical Physics, Session 68: Probing the Standard Model of Particle Interactions, (1998), pp. 281-539 [hep-ph/9806471] [INSPIRE]. 
[138] A.J. Buras, D. Guadagnoli and G. Isidori, On $\epsilon_{K}$ Beyond Lowest Order in the Operator Product Expansion, Phys. Lett. B $6 \mathbf{6 8}$ (2010) 309 [arXiv: 1002.3612] [InSPIRE].

[139] CKMfitter Group collaboration, CP violation and the CKM matrix: Assessing the impact of the asymmetric B factories, Eur. Phys. J. C 41 (2005) 1 [hep-ph/0406184] [InSPIRE].

[140] J.M. Pendlebury et al., Revised experimental upper limit on the electric dipole moment of the neutron, Phys. Rev. D 92 (2015) 092003 [arXiv: 1509.04411] [INSPIRE].

[141] C.A. Baker et al., An improved experimental limit on the electric dipole moment of the neutron, Phys. Rev. Lett. 97 (2006) 131801 [hep-ex/0602020] [INSPIRE].

[142] W.C. Griffith, M.D. Swallows, T.H. Loftus, M.V. Romalis, B.R. Heckel and E.N. Fortson, Improved Limit on the Permanent Electric Dipole Moment of Hg-199, Phys. Rev. Lett. 102 (2009) 101601 [arXiv:0901.2328] [INSPIRE].

[143] B. Graner, Y. Chen, E.G. Lindahl and B.R. Heckel, Reduced Limit on the Permanent Electric Dipole Moment of Hg199, Phys. Rev. Lett. 116 (2016) 161601 [Erratum ibid. 119 (2017) 119901] [arXiv: 1601.04339] [INSPIRE].

[144] M. Bishof et al., Improved limit on the ${ }^{225}$ Ra electric dipole moment, Phys. Rev. C 94 (2016) 025501 [arXiv: 1606.04931] [INSPIRE].

[145] T. Chupp, P. Fierlinger, M. Ramsey-Musolf and J. Singh, Electric dipole moments of atoms, molecules, nuclei, and particles, Rev. Mod. Phys. 91 (2019) 015001 [arXiv:1710.02504] [INSPIRE].

[146] C.-Y. Seng, J. de Vries, E. Mereghetti, H.H. Patel and M. Ramsey-Musolf, Nucleon electric dipole moments and the isovector parity- and time-reversal-odd pion-nucleon coupling, Phys. Lett. B 736 (2014) 147 [arXiv:1401.5366] [INSPIRE].

[147] M. Pospelov and A. Ritz, Neutron EDM from electric and chromoelectric dipole moments of quarks, Phys. Rev. D 63 (2001) 073015 [hep-ph/0010037] [INSPIRE].

[148] O. Lebedev, K.A. Olive, M. Pospelov and A. Ritz, Probing CP-violation with the deuteron electric dipole moment, Phys. Rev. D 70 (2004) 016003 [hep-ph/0402023] [INSPIRE].

[149] U. Haisch and A. Hala, Sum rules for CP-violating operators of Weinberg type, JHEP 11 (2019) 154 [arXiv: 1909.08955] [INSPIRE].

[150] N. Yamanaka and E. Hiyama, Weinberg operator contribution to the nucleon electric dipole moment in the quark model, Phys. Rev. D 103 (2021) 035023 [arXiv:2011.02531] [INSPIRE].

[151] T. Bhattacharya, V. Cirigliano, R. Gupta, H.-W. Lin and B. Yoon, Neutron Electric Dipole Moment and Tensor Charges from Lattice QCD, Phys. Rev. Lett. 115 (2015) 212002 [arXiv: 1506. 04196] [INSPIRE].

[152] PNDME collaboration, Iso-vector and Iso-scalar Tensor Charges of the Nucleon from Lattice QCD, Phys. Rev. D 92 (2015) 094511 [arXiv: 1506.06411] [INSPIRE].

[153] T. Bhattacharya, V. Cirigliano, S. Cohen, R. Gupta, H.-W. Lin and B. Yoon, Axial, Scalar and Tensor Charges of the Nucleon from 2+1+1-flavor Lattice QCD, Phys. Rev. D 94 (2016) 054508 [arXiv: 1606.07049] [INSPIRE].

[154] R. Gupta, Y.-C. Jang, B. Yoon, H.-W. Lin, V. Cirigliano and T. Bhattacharya, Isovector Charges of the Nucleon from 2+1+1-flavor Lattice QCD, Phys. Rev. D 98 (2018) 034503 [arXiv: 1806. 09006] [INSPIRE]. 
[155] R. Gupta, B. Yoon, T. Bhattacharya, V. Cirigliano, Y.-C. Jang and H.-W. Lin, Flavor diagonal tensor charges of the nucleon from (2+1+1)-flavor lattice QCD, Phys. Rev. D 98 (2018) 091501 [arXiv: 1808.07597] [INSPIRE].

[156] J. Bsaisou et al., Nuclear Electric Dipole Moments in Chiral Effective Field Theory, JHEP 03 (2015) 104 [Erratum ibid. 05 (2015) 083] [arXiv:1411.5804] [INSPIRE].

[157] N. Yamanaka and E. Hiyama, Enhancement of the CP-odd effect in the nuclear electric dipole moment of ${ }^{6}$ Li, Phys. Rev. C 91 (2015) 054005 [arXiv: 1503. 04446] [INSPIRE].

[158] JEDI collaboration, New method for a continuous determination of the spin tune in storage rings and implications for precision experiments, Phys. Rev. Lett. 115 (2015) 094801 [arXiv: 1504.00635] [INSPIRE].

[159] L.I. Schiff, Measurability of Nuclear Electric Dipole Moments, Phys. Rev. 132 (1963) 2194 [INSPIRE].

[160] V.F. Dmitriev and R.A. Sen'kov, Schiff moment of the mercury nucleus and the proton dipole moment, Phys. Rev. Lett. 91 (2003) 212303 [nucl-th/0306050] [INSPIRE].

[161] J.H. de Jesus and J. Engel, Time-reversal-violating Schiff moment of Hg-199, Phys. Rev. C 72 (2005) 045503 [nucl-th/0507031] [INSPIRE].

[162] S. Ban, J. Dobaczewski, J. Engel and A. Shukla, Fully self-consistent calculations of nuclear Schiff moments, Phys. Rev. C 82 (2010) 015501 [arXiv: 1003.2598] [INSPIRE].

[163] V.A. Dzuba, V.V. Flambaum and S.G. Porsev, Calculation of P,T-odd electric dipole moments for diamagnetic atoms Xe-129, Yb-171, Hg-199, Rn-211, and Ra-225, Phys. Rev. A 80 (2009) 032120 [arXiv: 0906.5437] [INSPIRE].

[164] J. Engel, M.J. Ramsey-Musolf and U. van Kolck, Electric Dipole Moments of Nucleons, Nuclei, and Atoms: The Standard Model and Beyond, Prog. Part. Nucl. Phys. 71 (2013) 21 [arXiv: 1303.2371] [INSPIRE].

[165] Y.T. Chien, V. Cirigliano, W. Dekens, J. de Vries and E. Mereghetti, Direct and indirect constraints on CP-violating Higgs-quark and Higgs-gluon interactions, JHEP 02 (2016) 011 [arXiv: 1510.00725] [INSPIRE].

[166] G. Chauhan, P.S.B. Dev, R.N. Mohapatra and Y. Zhang, Perturbativity constraints on $\mathrm{U}(1)_{B-L}$ and left-right models and implications for heavy gauge boson searches, JHEP 01 (2019) 208 [arXiv:1811.08789] [INSPIRE].

[167] S.G. Johnson, The nlopt nonlinear-optimization package, https://github.com/stevengj/nlopt.

[168] T.P. Runarsson and X. Yao, Search biases in constrained evolutionary optimization IEEE Trans. Syst. Man Cybern. C 35 (2005) 233.

[169] ATLAS collaboration, Search for new resonances in mass distributions of jet pairs using $139 \mathrm{fb}^{-1}$ of pp collisions at $\sqrt{s}=13 \mathrm{TeV}$ with the ATLAS detector, JHEP 03 (2020) 145 [arXiv: 1910.08447] [INSPIRE].

[170] A. Crivellin and M. Hoferichter, $\beta$ Decays as Sensitive Probes of Lepton Flavor Universality, Phys. Rev. Lett. 125 (2020) 111801 [arXiv:2002.07184] [INSPIRE].

[171] K. Cheung, W.-Y. Keung, C.-T. Lu and P.-Y. Tseng, Vector-like Quark Interpretation for the CKM Unitarity Violation, Excess in Higgs Signal Strength, and Bottom Quark Forward-Backward Asymmetry, JHEP 05 (2020) 117 [arXiv:2001.02853] [INSPIRE].

[172] B. Belfatto, R. Beradze and Z. Berezhiani, The CKM unitarity problem: A trace of new physics at the TeV scale?, Eur. Phys. J. C 80 (2020) 149 [arXiv: 1906.02714] [InSPIRE]. 
[173] G. D'Ambrosio, G.F. Giudice, G. Isidori and A. Strumia, Minimal flavor violation: An effective field theory approach, Nucl. Phys. B 645 (2002) 155 [hep-ph/0207036] [InSPIRE].

[174] S. Bruggisser, R. Schäfer, D. van Dyk and S. Westhoff, The Flavor of UV Physics, JHEP 05 (2021) 257 [arXiv:2101.07273] [INSPIRE].

[175] C. Grojean, M. Montull and M. Riembau, Diboson at the LHC vs LEP, JHEP 03 (2019) 020 [arXiv: 1810.05149] [INSPIRE].

[176] R. Aoude, T. Hurth, S. Renner and W. Shepherd, The impact of flavour data on global fits of the MFV SMEFT, JHEP 12 (2020) 113 [arXiv:2003.05432] [INSPIRE].

[177] J.W.F. Valle, Leptonic CP Violation and Left-right Symmetry, Phys. Lett. B 138 (1984) 155 [INSPIRE].

[178] J.F. Nieves, D. Chang and P.B. Pal, Electric Dipole Moment of the Electron in Left-right Symmetric Theories, Phys. Rev. D 33 (1986) 3324 [INSPIRE].

[179] B. Bajc, M. Nemevšek and G. Senjanović, Probing leptonic CP phases in LFV processes, Phys. Lett. B 684 (2010) 231 [arXiv:0911.1323] [INSPIRE].

[180] V. Cirigliano, A. Kurylov, M.J. Ramsey-Musolf and P. Vogel, Lepton flavor violation without supersymmetry, Phys. Rev. D 70 (2004) 075007 [hep-ph/0404233] [INSPIRE].

[181] C.-H. Lee, P.S. Bhupal Dev and R.N. Mohapatra, Natural TeV-scale left-right seesaw mechanism for neutrinos and experimental tests, Phys. Rev. D 88 (2013) 093010 [arXiv: 1309.0774] [INSPIRE].

[182] P. Duka, J. Gluza and M. Zrałek, Quantization and renormalization of the manifest left-right symmetric model of electroweak interactions, Annals Phys. 280 (2000) 336 [hep-ph/9910279] [INSPIRE].

[183] K. Kiers, M. Assis and A.A. Petrov, Higgs sector of the left-right model with explicit CP-violation, Phys. Rev. D 71 (2005) 115015 [hep-ph/0503115] [INSPIRE].

[184] E.E. Jenkins, A.V. Manohar and M. Trott, Renormalization Group Evolution of the Standard Model Dimension Six Operators I: Formalism and lambda Dependence, JHEP 10 (2013) 087 [arXiv:1308.2627] [INSPIRE].

[185] E.E. Jenkins, A.V. Manohar and M. Trott, Renormalization Group Evolution of the Standard Model Dimension Six Operators II: Yukawa Dependence, JHEP 01 (2014) 035 [arXiv:1310.4838] [INSPIRE].

[186] E.E. Jenkins, A.V. Manohar and P. Stoffer, Low-Energy Effective Field Theory below the Electroweak Scale: Anomalous Dimensions, JHEP 01 (2018) 084 [arXiv:1711.05270] [INSPIRE].

[187] K.K. Vos, H.W. Wilschut and R.G.E. Timmermans, Symmetry violations in nuclear and neutron $\beta$ decay, Rev. Mod. Phys. 87 (2015) 1483 [arXiv:1509.04007] [INSPIRE].

[188] A.V. Manohar and M.B. Wise, Heavy quark physics, vol. 10 (2000) [INSPIRE].

[189] A. Sirlin, Large $m(W), m(Z)$ Behavior of the O(alpha) Corrections to Semileptonic Processes Mediated by W, Nucl. Phys. B 196 (1982) 83 [inSPIRE].

[190] Fermilab Lattice and MiLC collaborations, Update of $\left|V_{c b}\right|$ from the $\bar{B} \rightarrow D^{*} \ell \bar{\nu}$ form factor at zero recoil with three-flavor lattice QCD, Phys. Rev. D 89 (2014) 114504 [arXiv:1403.0635] [INSPIRE]. 
[191] MILC collaboration, $B \rightarrow D \ell \nu$ form factors at nonzero recoil and $\left|V_{c b}\right|$ from 2+1-flavor lattice QCD, Phys. Rev. D 92 (2015) 034506 [arXiv: 1503.07237] [InSPIRE].

[192] C.W. Bauer, Z. Ligeti, M. Luke and A.V. Manohar, B decay shape variables and the precision determination of $|V(c b)|$ and $m(b)$, Phys. Rev. D 67 (2003) 054012 [hep-ph/0210027] [INSPIRE].

[193] P. Gambino, B semileptonic moments at NNLO, JHEP 09 (2011) 055 [arXiv:1107.3100] [INSPIRE].

[194] P. Gambino and C. Schwanda, Inclusive semileptonic fits, heavy quark masses, and $V_{c b}$, Phys. Rev. D 89 (2014) 014022 [arXiv:1307.4551] [INSPIRE].

[195] A. Alberti, P. Gambino, K.J. Healey and S. Nandi, Precision Determination of the Cabibbo-Kobayashi-Maskawa Element $V_{c b}$, Phys. Rev. Lett. 114 (2015) 061802 [arXiv:1411.6560] [INSPIRE].

[196] Z.-R. Huang, E. Kou, C.-D. Lü and R.-Y. Tang, Un-binned Angular Analysis of $B \rightarrow D^{*} \ell \nu_{\ell}$ and the Right-handed Current, arXiv:2106.13855 [INSPIRE].

[197] B. Dassinger, R. Feger and T. Mannel, Complete Michel Parameter Analysis of inclusive semileptonic $b \rightarrow c$ transition, Phys. Rev. D 79 (2009) 075015 [arXiv:0803.3561] [InSPIRE].

[198] R. Feger, T. Mannel, V. Klose, H. Lacker and T. Luck, Limit on a Right-Handed Admixture to the Weak $b \rightarrow c$ Current from Semileptonic Decays, Phys. Rev. D 82 (2010) 073002 [arXiv: 1003.4022] [INSPIRE].

[199] C.W. Bauer, Z. Ligeti and M.E. Luke, Precision determination of $\left|V_{u b}\right|$ from inclusive decays, Phys. Rev. D 64 (2001) 113004 [hep-ph/0107074] [InSPIRE].

[200] B.O. Lange, M. Neubert and G. Paz, Theory of charmless inclusive B decays and the extraction of $V_{u b}$, Phys. Rev. D 72 (2005) 073006 [hep-ph/0504071] [INSPIRE].

[201] W. Detmold, C. Lehner and S. Meinel, $\Lambda_{b} \rightarrow p \ell^{-} \bar{\nu}_{\ell}$ and $\Lambda_{b} \rightarrow \Lambda_{c} \ell^{-} \bar{\nu}_{\ell}$ form factors from lattice QCD with relativistic heavy quarks, Phys. Rev. D 92 (2015) 034503 [arXiv: 1503.01421] [INSPIRE].

[202] LHCb collaboration, Determination of the quark coupling strength $\left|V_{u b}\right|$ using baryonic decays, Nature Phys. 11 (2015) 743 [arXiv:1504.01568] [INSPIRE].

[203] W. Altmannshofer and D.M. Straub, Cornering New Physics in $b \rightarrow s$ Transitions, JHEP 08 (2012) 121 [arXiv: 1206.0273] [InSPIRE].

[204] W. Altmannshofer, P. Paradisi and D.M. Straub, Model-Independent Constraints on New Physics in $b \rightarrow s$ Transitions, JHEP 04 (2012) 008 [arXiv:1111.1257] [InSPIRE].

[205] G.C. Branco, L. Lavoura and J.P. Silva, CP Violation, Int. Ser. Monogr. Phys. 103 (1999) 1.

[206] T. Hurth, E. Lunghi and W. Porod, Untagged $\bar{B} \rightarrow X_{s+d} \gamma$ CP asymmetry as a probe for new physics, Nucl. Phys. B 704 (2005) 56 [hep-ph/0312260] [InSPIRE].

[207] M. Misiak et al., Estimate of $\mathcal{B}\left(\bar{B} \rightarrow X_{s} \gamma\right)$ at $O\left(\alpha_{s}^{2}\right)$, Phys. Rev. Lett. 98 (2007) 022002 [hep-ph/0609232] [inSPIRE].

[208] M. Misiak et al., Updated NNLO QCD predictions for the weak radiative B-meson decays, Phys. Rev. Lett. 114 (2015) 221801 [arXiv: 1503.01789] [INSPIRE].

[209] M. Czakon, P. Fiedler, T. Huber, M. Misiak, T. Schutzmeier and M. Steinhauser, The $\left(Q_{7}, Q_{1,2}\right)$ contribution to $\bar{B} \rightarrow X_{s} \gamma$ at $\mathcal{O}\left(\alpha_{\mathrm{s}}^{2}\right)$, JHEP 04 (2015) 168 [arXiv:1503.01791] [INSPIRE]. 
[210] M. Benzke, S.J. Lee, M. Neubert and G. Paz, Long-Distance Dominance of the CP Asymmetry in $B \rightarrow X_{s, d}+\gamma$ Decays, Phys. Rev. Lett. 106 (2011) 141801 [arXiv:1012.3167] [INSPIRE].

[211] A. Paul and D.M. Straub, Constraints on new physics from radiative B decays, JHEP 04 (2017) 027 [arXiv: 1608.02556] [INSPIRE].

[212] P. Ball and R. Zwicky, Time-dependent CP Asymmetry in $B \rightarrow K^{*} \gamma$ as a (Quasi) Null Test of the Standard Model, Phys. Lett. B 642 (2006) 478 [hep-ph/0609037] [InSPIRE].

[213] P. Ball, G.W. Jones and R. Zwicky, $B \rightarrow V \gamma$ beyond QCD factorisation, Phys. Rev. D 75 (2007) 054004 [hep-ph/0612081] [INSPIRE].

[214] A.J. Buras and R. Fleischer, Quark mixing, CP-violation and rare decays after the top quark discovery, Adv. Ser. Direct. High Energy Phys. 15 (1998) 65 [hep-ph/9704376] [INSPIRE].

[215] M. Artuso, G. Borissov and A. Lenz, CP violation in the $B_{s}^{0}$ system, Rev. Mod. Phys. 88 (2016) 045002 [Addendum ibid. 91 (2019) 049901] [arXiv: 1511.09466] [INSPIRE].

[216] I. Baum, V. Lubicz, G. Martinelli, L. Orifici and S. Simula, Matrix elements of the electromagnetic operator between kaon and pion states, Phys. Rev. D 84 (2011) 074503 [arXiv: 1108.1021] [INSPIRE]. 